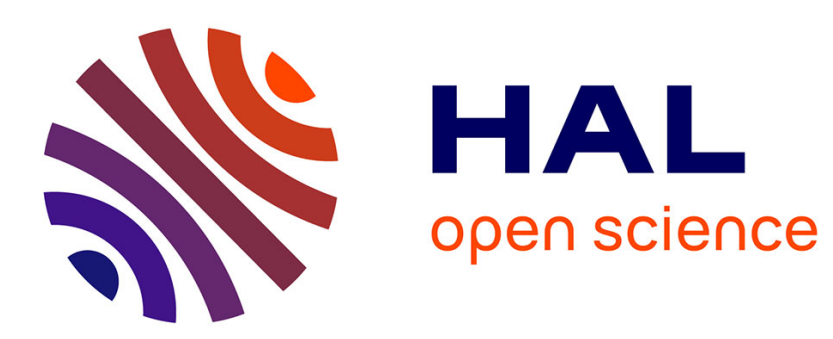

\title{
Boundary null-controllability of 1-D coupled parabolic systems with Kirchhoff-type condition
}

Kuntal Bhandari, Franck Boyer, Víctor Hernández-Santamaría

\section{To cite this version:}

Kuntal Bhandari, Franck Boyer, Víctor Hernández-Santamaría. Boundary null-controllability of 1D coupled parabolic systems with Kirchhoff-type condition. Mathematics of Control, Signals, and Systems, 2021, 33 (3), pp.413-471. 10.1007/s00498-021-00285-z . hal-02748405v3

\section{HAL Id: hal-02748405 \\ https://hal.science/hal-02748405v3}

Submitted on 12 Feb 2021

HAL is a multi-disciplinary open access archive for the deposit and dissemination of scientific research documents, whether they are published or not. The documents may come from teaching and research institutions in France or abroad, or from public or private research centers.
L'archive ouverte pluridisciplinaire HAL, est destinée au dépôt et à la diffusion de documents scientifiques de niveau recherche, publiés ou non, émanant des établissements d'enseignement et de recherche français ou étrangers, des laboratoires publics ou privés. 


\title{
Boundary null-controllability of 1-D coupled parabolic systems with Kirchhoff-type conditions
}

\author{
Kuntal Bhandari • Franck Boyer • \\ Víctor Hernández-Santamaría
}

Received: date / Accepted: date

\begin{abstract}
The main concern of this article is to investigate the boundary controllability of some $2 \times 2$ one-dimensional parabolic systems with both the interior and boundary couplings: the interior coupling is chosen to be linear with constant coefficient while the boundary one is considered by means of some Kirchhoff-type condition at one end of the domain. We consider here the Dirichlet boundary control acting only on one of the two state components at the other end of the domain. In particular, we show that the controllability properties change depending on which component of the system the control is being applied. Regarding this, we point out that the choices of interior coupling coefficient and the Kirchhoff parameter play a crucial role to deduce the positive or negative controllability results.

Further to this, we pursue a numerical study based on the well-known penalized HUM approach. We make some discretization for a general interior-boundary coupled parabolic system, mainly to incorporate the effects of the boundary couplings into the discrete setting. This allows us to illustrate our theoretical results
\end{abstract}

The work of the third author was partially supported by the Labex CIMI (Centre International de Mathématiques et d'Informatique), ANR-11-LABX-0040-CIMI, France.

K. Bhandari

Institut de Mathématiques de Toulouse, UMR 5219,

Université Paul Sabatier,

31062 Toulouse Cedex 09, France

E-mail: kuntal.bhandari1993@gmail.com

F. Boyer

Institut de Mathématiques de Toulouse, UMR 5219,

Université Paul Sabatier,

Institut Universitaire de France,

31062 Toulouse Cedex 09, France

E-mail: franck.boyer@math.univ-toulouse.fr

V. Hernández-Santamaría

Instituto de Matemáticas,

Universidad Nacional Autónoma de México, Circuito Exterior C.U.,

C.P. 04510 CDMX, México

E-mail: victor.santamaria@im.unam.mx 
as well as to experiment some more examples which fit under the general framework, for instance a similar system with a Neumann boundary control on either one of the two components.

Keywords Boundary control · Parabolic systems · Carleman estimate - Moments method $\cdot$ Spectral analysis $\cdot$ Kirchhoff conditions

Mathematics Subject Classification (2010) 35K20 - 93B05 -93B07 -93B60

\section{Introduction}

1.1 Motivation and the problem under study

In this article, we discuss about the boundary null-controllability of some $2 \times 2$ one-dimensional parabolic systems coupled through interior as well as boundary with one control force. We write down the following prototype of $2 \times 2$ general coupled parabolic control system that contains both kinds of couplings,

$$
\begin{cases}\partial_{t} y+\mathcal{A} y+\mathcal{M}_{\text {coup }} y=0 & \text { in }(0, T) \times(0,1), \\ \mathcal{D}_{0} y(t, 0)+\mathcal{N}_{0} \frac{\partial y}{\partial \nu_{\mathcal{A}}}(t, 0)=B v(t) & \text { in }(0, T), \\ \mathcal{D}_{1} y(t, 1)+\mathcal{N}_{1} \frac{\partial y}{\partial \nu_{\mathcal{A}}}(t, 1)=0 & \text { in }(0, T), \\ y(0, \cdot)=y_{0}(\cdot) & \text { in }(0,1),\end{cases}
$$

where $y:=\left(y_{1}, y_{2}\right)$ is the unknown and $y_{0}:=\left(y_{0,1}, y_{0,2}\right)$ is the initial data from some suitable Hilbert space and $\mathcal{M}_{\text {coup }}, \mathcal{D}_{j}, \mathcal{N}_{j} \in M_{2 \times 2}(\mathbb{R})(j=0,1)$. The scalar input $v$ is assumed to act as a control on the boundary point $x=0$ through some non-zero vector $B \in \mathbb{R}^{2}$. Further, we choose the operator $\mathcal{A}$ with the formal expression

$$
\mathcal{A}:=\left(\begin{array}{cc}
-\partial_{x}\left(\gamma_{1} \partial_{x}\right) & 0 \\
0 & -\partial_{x}\left(\gamma_{2} \partial_{x}\right)
\end{array}\right),
$$

where the diffusion coefficients $\gamma_{1}, \gamma_{2}$ are chosen in such a way that

$$
\gamma_{i} \in \mathcal{C}^{1}([0,1]) \text { with } \gamma_{\min }:=\inf _{[0,1]}\left\{\gamma_{i}, i=1,2\right\}>0
$$

The domain of $\mathcal{A}$ can be defined as

$$
D(\mathcal{A}):=\left\{u \in\left(H^{2}(0,1)\right)^{2} \mid \mathcal{D}_{0} u(0)+\mathcal{N}_{0} \frac{\partial u}{\partial \nu_{\mathcal{A}}}(0)=0, \mathcal{D}_{1} u(1)+\mathcal{N}_{1} \frac{\partial u}{\partial \nu_{\mathcal{A}}}(1)=0\right\}
$$

with the conormal derivative operator $\frac{\partial}{\partial \nu_{\mathcal{A}}}:=\left(\gamma_{1} \frac{\partial}{\partial \nu}, \gamma_{2} \frac{\partial}{\partial \nu}\right)$ ( $\nu$ is the normal vector) on the boundary points $x=0,1$.

The main point here is that we consider the interior coupling by means of some matrix $\mathcal{M}_{\text {coup }}$ and the boundary couplings via the coefficient matrices $\mathcal{D}_{j}, \mathcal{N}_{j}$, for $j=0,1$. We further impose the following assumptions.

Assumption 1 For each $j \in\{0,1\}$, 
1. The $2 \times 4$ matrix $\left(\mathcal{D}_{j}, \mathcal{N}_{j}\right)$ has the maximal rank.

2. The matrix $\mathcal{D}_{j} \mathcal{N}_{j}^{*}$ is self-adjoint.

The first assumption ensures the sufficient number of boundary conditions in (1.1), whereas the second one is important for the differential operator $\mathcal{A}$ defined by (1.2) to be self-adjoint in its domain $D(\mathcal{A}),(1.4)$.

From the point of bibliographic comments, we first want to mention that the parabolic boundary control systems with less number of controls than the equations can be really intricate to study in various situations and that there is lack of enough mathematical tools to tackle with these systems. In fact, unlike the scalar problems the boundary controllability for such systems is no longer equivalent with the distributed one, as it has been proven for instance in [26]. Moreover, the very powerful Carleman technique is often inefficient in that context.

Among some very fascinating works on (interior) coupled control systems, we point out [26] where the authors proved a necessary and sufficient condition for boundary null-controllability of some $2 \times 2$ coupled parabolic system with single Dirichlet control. Also, the reference [3] is dedicated to prove the controllability to the trajectories of an $n \times n$ parabolic system when $m<n$ Dirichlet controls are exerted on a part of the boundary; the authors actually proved a general Kalman condition which is a necessary and sufficient condition for their problem.

As per our knowledge, most of the boundary controllability results for a system with less controls than the equations are in 1-D. This is mainly due to the fact that the spectral analysis of the associated adjoint elliptic operator might give the advantage to develop the so-called moments equations to construct a control. In this regard, we mention that some multi-D (in cylindrical geometry) results have been developed in $[8,2]$, which need a sharp estimate of the control cost for the associated 1-D problem and a Lebeau-Robbiano spectral inequality for higher dimensions. We also quote [1] where the null-controllability of some symmetric wave-type system (as well as parabolic and Schrödinger-type systems) has been analyzed with one control in any dimension, provided that the control region satisfies the Geometric Control Condition. Finally, we refer to [4] where the authors made a survey of several recent results concerning the controllability of coupled parabolic systems.

Let us come to the point of boundary couplings. We mention that several systems with boundary couplings (let say without any control for the moment) use to appear when one considers some parabolic system on a metric graph; we quote here very few of those, e.g., $[37,32,10]$. In this context, we must say that there are diverse applications of PDEs systems on metric graphs in physics, chemistry or biology, see for instance $[6,16]$ and the references therein.

Concerning the controllability issues on metric graphs, we first address $[22$, Chapters 6,8] where the authors discussed some controllability results of wave, heat and Schrödinger systems considered in some network in the case when some control(s) is (are) exerted on some of the vertices; see also the survey paper [7]. Furthermore, the authors of [17] proved some boundary null-controllability results for a linear Kuramoto-Sivashinsky equation on star-shaped trees with Dirichlet or Neumann boundary controls. We also refer to the very recent works [19] and [18] which contain some boundary controllability results for $\mathrm{KdV}$ equations on a treeshaped and star-shaped network, respectively. Last but not the least, it is worth 
to quote that a necessary and sufficient condition for approximate controllability of two 1-D wave systems has been developed in [21].

In most of the known cases, one cannot arbitrarily impose an interior coupling to the system of differential equations that are considered on a metric graph. The reason is that each edge corresponds to only one scalar differential equation with respect to one unknown. In this case, we point out that the only interaction between the unknowns occurs at the vertices.

We now come to the case of (1.1), where one observes that the main difference between this kind of systems and the systems that generally arise on graphs is: unlike the situation on graph, we have here the possibility of considering both the boundary and interior couplings, and thus, it is reasonable to expect different kind of influence of the control to the concerned system.

At that moment, two types of difficulties may arise while dealing with the general system like (1.1). As mentioned earlier, the Carleman technique is often inefficient in the framework of a boundary control system. Beside this obstruction, there occurs a change in the spectral analysis of the adjoint elliptic operator associated with the parabolic system, since this kind of operator is normally non self-adjoint in nature due to the presence of the interior coupling $\mathcal{M}_{\text {coup }}$. Thus, it is not so straightforward to apply a moments technique to the general system (1.1). Moreover, in the framework of the system (1.1) with Assumption 1, there are examples of negative controllability results also.

Remark 1.1 (Some negative controllability results) It can be shown that, a linear coupled system in the cascade form, with either $\mathcal{D}_{j}=\left(\begin{array}{ll}1 & 0 \\ 0 & 0\end{array}\right), \mathcal{N}_{j}=\left(\begin{array}{ll}0 & 0 \\ 0 & 1\end{array}\right)$ or, $\mathcal{D}_{j}=\left(\begin{array}{ll}0 & 0 \\ 0 & 1\end{array}\right), \mathcal{N}_{j}=\left(\begin{array}{ll}1 & 0 \\ 0 & 0\end{array}\right)(j=0,1)$, and for $B=\left(\begin{array}{l}1 \\ 0\end{array}\right)$, is not even approximately controllable; see for instance [12, Remark 2.17].

Due to this indistinct phenomena, in our present work, we will mainly focus on some particular class of systems which fit in the framework of (1.1).

1.2 Main concerns of the paper

We hereby choose the interior coupling

$$
\mathcal{M}_{\text {coup }}=\mathcal{M}_{a}:=\left(\begin{array}{ll}
0 & 0 \\
a & 0
\end{array}\right)
$$

for some $a \in \mathbb{R}$, and the boundary coefficient matrices,

$$
\begin{array}{r}
\mathcal{D}_{0}=I_{2 \times 2}, \quad \mathcal{N}_{0}=0_{2 \times 2}, \\
\mathcal{D}_{1}=\left(\begin{array}{cc}
1 & -1 \\
\alpha & 0
\end{array}\right), \quad \mathcal{N}_{1}=\left(\begin{array}{ll}
0 & 0 \\
1 & 1
\end{array}\right),
\end{array}
$$

for some $\alpha \geq 0$. In what follows, we have the systems below. 
Two Dirichlet control systems. Under the consideration of (1.5) and (1.6a)-(1.6b), we write down the following systems.

$$
\begin{cases}\partial_{t} y_{1}-\partial_{x}\left(\gamma_{1} \partial_{x} y_{1}\right)=0 & \text { in }(0, T) \times(0,1), \\ \partial_{t} y_{2}-\partial_{x}\left(\gamma_{2} \partial_{x} y_{2}\right)+a y_{1}=0 & \text { in }(0, T) \times(0,1), \\ y_{1}(t, 1)=y_{2}(t, 1) & \text { in }(0, T), \\ \gamma_{1}(1) \partial_{x} y_{1}(t, 1)+\gamma_{2}(1) \partial_{x} y_{2}(t, 1)+\alpha y_{1}(t, 1)=0 & \text { in }(0, T), \\ y_{1}(0, \cdot)=y_{0,1}(\cdot), \quad y_{2}(0, \cdot)=y_{0,2}(\cdot) & \text { in }(0,1),\end{cases}
$$

with a Dirichlet control at the left end point either on the second or first component depending on the choices of $B=\left(\begin{array}{l}0 \\ 1\end{array}\right)$ or $\left(\begin{array}{l}1 \\ 0\end{array}\right)$, that is to say

$$
\begin{array}{rlll}
\text { either } & y_{1}(t, 0)=0, \quad y_{2}(t, 0)=v(t) & \text { in }(0, T), \\
\text { or } & y_{1}(t, 0)=v(t), \quad y_{2}(t, 0)=0 & \text { in }(0, T) .
\end{array}
$$

We have chosen here a general Kirchhoff condition at the boundary point $x=1$ (the standard one is with $\alpha=0$ ). It is notable that the Kirchhoff-type conditions generally appear widely in physics, electrical engineering and in various biological models, see for instance $[33,16]$. In the framework of control theory, the usual type of system that has already been studied in the literature is the case when there is no interior coupling (i.e., $a=0$ ) and with the standard Kirchhoff condition, see for instance $[22$, Ch. 8] and [7,17] (mentioned earlier). We also recall that, a controllability result of some $2 \times 2$ parabolic system without any interior coupling has been addressed in [9, Remark 3.6], where a Kirchhoff condition with $\alpha=0$ has been considered.

But observe that when $a=0$, the two control systems (1.7)-(1.8a) and (1.7)(1.8b) are exactly the same.

Now, as soon as one considers some interior coupling coefficient $a \neq 0$, the control systems (1.7)-(1.8a) and (1.7)-(1.8b) are certainly different in nature and the choices of the component on which we exert the control really have an influence to the controllability issues. In fact, in the second situation (when we put the control on the first component), the particular value of $(\alpha, a)$ is very crucial to conclude the positive or negative controllability phenomena.

Before going to further details, we prescribe the following notations that will be used in our paper.

Notations. Throughout the paper we shall make use of following notations. The inner product and norm in the scalar space $L^{2}(0,1)$ will be simply denoted by $(\cdot, \cdot)_{L^{2}}$ and $\|\cdot\|_{L^{2}}$ respectively. We also denote the space $E:=\left(L^{2}(0,1)\right)^{2}$, its inner product and the norm by $(\cdot, \cdot)_{E}$ and $\|\cdot\|_{E}$ respectively. Moreover, we use the notation $\langle\cdot, \cdot\rangle_{X^{\prime}, X}$ to express the duality pair between a space $X$ and its dual $X^{\prime}$. Beside this, we sometimes write $\langle\cdot, \cdot\rangle_{U}$ with $U=\mathbb{R}^{d}$ or $\mathbb{C}^{d}, d \geq 1$, to specify the usual inner product in $U$.

Further, we declare $\mathbb{R}^{*}:=\mathbb{R} \backslash\{0\}$ and $\mathbb{R}_{0}^{+}:=\mathbb{R}^{+} \cup\{0\}$, where $\mathbb{R}^{+}$denotes the set of all positive real numbers.

We use the letter $C$ and subsequently $\widetilde{C}, C^{\prime}, C^{\prime \prime}$ to denote some positive constants (those may vary from line to line) which do possibly depend on $\gamma_{1}, \gamma_{2}, \alpha, a$ 
but not on $T$ and $y_{0}$. Sometimes, we shall express some constants by $C_{p_{1}, p_{2}, \cdots, p_{n}}$ to specify its dependency on the quantities $p_{1}, p_{2}, \cdots, p_{n}$.

We often use the symbol $M^{*}$ to denote the adjoint of a matrix or an operator $M$.

Two main theorems. Let us present the main results regarding the null-controllability of our systems (1.7)-(1.8).

1. Case 1. To show the boundary null-controllability of the problem (1.7)-(1.8a), that is when we consider the control applied on $y_{2}$, we prove a suitable observability inequality, which in turn is consequence of a suitable Carleman estimate, detailed in Section 3.1. Our main theorem is the following.

Theorem 1.2 Let any $(\alpha, a) \in \mathbb{R}_{0}^{+} \times \mathbb{R}$ and $T>0$ be given. Then, for any $y_{0} \in$ $\mathcal{H}_{-\alpha}$, there exists a null-control $v \in L^{2}(0, T ; \mathbb{R})$ for the problem $(1.7)-(1.8 \mathrm{a})$, that satisfies the estimate

$$
\|v\|_{L^{2}(0, T)} \leq C e^{C / T}\left\|y_{0}\right\|_{\mathcal{H}_{-\alpha}},
$$

with the constant $C:=C\left(\gamma_{1}, \gamma_{2}, \alpha, a\right)>0$ that does not depend on $T>0$ and $y_{0}$.

The space $\mathcal{H}_{-\alpha}$ will be introduced in Section 2.1 .

2. Case 2. Surprisingly, it appears that the Carleman strategy cannot be applied to the system $(1.7)-(1.8 \mathrm{~b})$, that is when we consider our control to act on $y_{1}$. This is because, the source integral due to the interior coupling in our Carleman estimate cannot be controlled by the boundary observation term with our choices of weight functions (defined later in Section 3.1); the exact technical point behind this will be specified in Remark 3.5.

Due to this obstacle, the next immediate idea is to investigate the spectral properties of the adjoint elliptic operator associated with our parabolic system and try to develop the moments method to construct a control by hand. In this case, we restrict the diffusion coefficients $\gamma_{1}=\gamma_{2}=1$ to understand the situation more concretely. Indeed, by studying the spectral analysis, we observe that the choices of coupling coefficient $a$ and the boundary parameter $\alpha$ have a genuine influence to the controllability of $(1.7)-(1.8 \mathrm{~b})$, which is not alike the previous case, as per Theorem 1.2.

In fact, this tells us that we cannot hope to find a good observability inequality using the Carleman estimate in the case of (1.7)-(1.8b).

This is quite surprising as we know that, for a cascade system the usual business is to apply a boundary control on the first component $y_{1}$ to study the controllability issue. Of course, in that case, there is no direct influence of the control to the equation of $y_{2}$, and here comes the role of the interior coupling: it acts as an indirect control to $y_{2}$. But, as explained above, in the case of $(1.7)-(1.8 \mathrm{~b})$, we have the opposite phenomena.

Let us state more precisely the controllability theorem we obtain.

Theorem 1.3 We fix $\gamma_{1}=\gamma_{2}=1$. Then, there exists a set $\mathcal{R} \subset \mathbb{R}_{0}^{+} \times \mathbb{R}^{*}$ of null measure such that

(a) for each pair $(\alpha, a) \notin \mathcal{R}$, there is a null-control to the problem (1.7)-(1.8b), for any given data $y_{0} \in \mathcal{H}_{-\alpha}$, 
(b) for each pair $(\alpha, a) \in \mathcal{R}$, there exists a subspace $\mathcal{Y}_{\alpha, a} \subset \mathcal{H}_{-\alpha}$ of co-dimension 1 , such that there exists a null-control to the problem $(1.7)-(1.8 \mathrm{~b})$, if and only if $y_{0} \in \mathcal{Y}_{\alpha, a}$.

In addition, in the controllable cases we can choose such a null-control $v \in L^{2}(0, T ; \mathbb{R})$ that satisfies the bound

$$
\|v\|_{L^{2}(0, T)} \leq C_{\alpha, a} e^{C_{\alpha, a} / T}\left\|y_{0}\right\|_{\mathcal{H}_{-\alpha}}
$$

where $C_{\alpha, a}>0$ is independent on $T>0$ and $y_{0}$.

The set $\mathcal{R}$ and the spaces $\mathcal{Y}_{\alpha, a}$ will be specified later, namely in (4.29) and (4.31) in Section 4.2.1. The set $\mathcal{R}$ actually corresponds to the parameters $(a, \alpha)$ for which our operator has (at least) one non observable eigenmode. For each such pair, $\mathcal{Y}_{\alpha, a}$ will be the orthogonal of those non observable eigenmodes.

Remark 1.4 In the case when $(\alpha, a) \in \mathcal{R}$, the problem $(1.7)-(1.8 \mathrm{~b})$ is not even approximately controllable if we choose our initial data $y_{0} \notin \mathcal{Y}_{\alpha, a}$.

Some numerical studies. Beside the theoretical ones, we also pursue some numerical studies in this paper. More precisely, we shall present a discretization (in terms of finite-differences) of the general system (1.1) under the Assumption 1 in Section 5.2. This will allow us to illustrate our theoretical results as well as some more examples in the framework of (1.1), in terms of the notable penalized Hilbert Uniqueness Method (HUM) ([30,14]).

In this regard, we must mention that several authors have utilized the penalized HUM technique to clarify various controllability issues related to the parabolic systems. For instance, the authors in [15] dealt with a numerical study of insensitizing control problems for parabolic semilinear equation and in [13] the controllability of some 1-D fractional heat equation has been analyzed. We also quote [5], where some partial controllability results have been illustrated with help of this technique.

Concerning the numerical studies for parabolic systems, in most of the known cases no boundary coupling has been taken into account. In our case, we will introduce a discretization methodology for the interior-boundary coupled parabolic system (1.1) mainly to incorporate the effects of the boundary couplings into our discrete setting.

Remark 1.5 (Neumann control problems) By taking into consideration $\mathcal{D}_{0}=0_{2 \times 2}$ and $\mathcal{N}_{0}=I_{2 \times 2}$ along with (1.6b), one has two Neumann boundary control systems, that is the system (1.7) along with the following two different kinds of Neumann controls at $x=0$, depending on the choices of $B=\left(\begin{array}{l}0 \\ 1\end{array}\right)$ or $\left(\begin{array}{l}1 \\ 0\end{array}\right)$, that is

$$
\begin{array}{rlll}
\text { either } & \partial_{x} y_{1}(t, 0)=0, \quad \gamma_{2}(0) \partial_{x} y_{2}(t, 0)=v(t) & \text { in }(0, T), \\
\text { or } & \gamma_{1}(0) \partial_{x} y_{1}(t, 0)=v(t), \quad \partial_{x} y_{2}(t, 0)=0 & \text { in }(0, T) .
\end{array}
$$

We will not study this case theoretically, rather we will investigate this from a numerical point of view in Section 5.3.2. 
1.3 Paper organization

We shall organize our paper in the following way:

- In Section 2, we shall present some functional setting and the well-posedness results related to our systems (1.7)-(1.8). To this end, we formulate the nullcontrol problems concerned with those systems.

- Thereafter, Section 3 will be devoted to obtain the null-controllability of the system (1.7)-(1.8a), i.e., proving the Theorem 1.2. In this case, we actually prove a Carleman estimate to attain a suitable observability inequality.

- Coming to the Section 4, we first develop some spectral analysis of the adjoint elliptic operator associated with the control system (1.7)-(1.8b) in Section 4.1 and then focus on finding the lower bounds of the observation estimates and upper bounds of the eigen-estimates in Section 4.2. This, along with a suitable bi-orthogonal family to the exponentials, will lead us to construct a control via the moments method in Section 4.3 for the system (1.7)-(1.8b) (i.e., proving the Theorem 1.3).

- Finally, some numerical studies along with several experiments will be pursued in Section 5.

\section{General settings and the well-posedness of the systems}

This section is devoted to study the well-posedness result of the boundary control systems (1.7)-(1.8). Also, we formulate the control problems related to those systems.

2.1 The system with homogeneous Dirichlet data

Let us begin with the following coupled parabolic system with a Kirchhoff-type condition at the right end point and the homogeneous Dirichlet conditions at the left end point.

$$
\begin{cases}\partial_{t} y_{1}-\partial_{x}\left(\gamma_{1} \partial_{x} y_{1}\right)=f_{1} & \text { in }(0, T) \times(0,1), \\ \partial_{t} y_{2}-\partial_{x}\left(\gamma_{2} \partial_{x} y_{2}\right)+a y_{1}=f_{2} & \text { in }(0, T) \times(0,1), \\ y_{1}(t, 0)=y_{2}(t, 0)=0 & \text { in }(0, T), \\ y_{1}(t, 1)=y_{2}(t, 1) & \text { in }(0, T), \\ \gamma_{1}(1) \partial_{x} y_{1}(t, 1)+\gamma_{2}(1) \partial_{x} y_{2}(t, 1)+\alpha y_{1}(t, 1)=0 & \text { in }(0, T), \\ y_{1}(0, \cdot)=y_{0,1}(\cdot), \quad y_{2}(0, \cdot)=y_{0,2}(\cdot) & \text { in }(0,1),\end{cases}
$$

where regularity of $y_{0}=\left(y_{0,1}, y_{0,2}\right)$ and $f=\left(f_{1}, f_{2}\right)$ will be specified later.

We introduce the self-adjoint and positive elliptic operator $\mathcal{A}_{\alpha}$, corresponding to the above system without interior coupling with its formal expression

$$
\mathcal{A}_{\alpha}:=\left(\begin{array}{cc}
-\partial_{x}\left(\gamma_{1} \partial_{x}\right) & 0 \\
0 & -\partial_{x}\left(\gamma_{2} \partial_{x}\right)
\end{array}\right)
$$


with its domain

$$
\begin{gathered}
D\left(\mathcal{A}_{\alpha}\right):=\left\{u=\left(u_{1}, u_{2}\right) \in\left(H^{2}(0,1)\right)^{2} \mid u_{1}(0)=u_{2}(0)=0, u_{1}(1)=u_{2}(1)\right. \\
\left.\gamma_{1}(1) u_{1}^{\prime}(1)+\gamma_{2}(1) u_{2}^{\prime}(1)+\alpha u_{1}(1)=0\right\}
\end{gathered}
$$

Let us consider the space $\mathcal{H}_{\alpha}:=D\left(\mathcal{A}_{\alpha}^{1 / 2}\right)$ as a completion of $D\left(\mathcal{A}_{\alpha}\right)$ with respect to the norm

$$
\|u\|_{\mathcal{H}_{\alpha}}:=\left(\mathcal{A}_{\alpha} u, u\right)_{E}^{1 / 2}=\left(\sum_{i=1}^{2} \int_{0}^{1} \gamma_{i}(x)\left|u_{i}^{\prime}(x)\right|^{2} d x+\alpha\left|u_{1}(1)\right|^{2}\right)^{1 / 2}, \forall u \in D\left(\mathcal{A}_{\alpha}\right),
$$

and one can prove that

$$
\mathcal{H}_{\alpha}=\left\{u=\left(u_{1}, u_{2}\right) \in\left(H^{1}(0,1)\right)^{2} \mid u_{1}(0)=u_{2}(0)=0, u_{1}(1)=u_{2}(1)\right\} .
$$

Moreover, we denote the dual space of $\mathcal{H}_{\alpha}$ by $\mathcal{H}_{-\alpha}$ with respect to the pivot space E.

Remark 2.1 Although, the space $\mathcal{H}_{\alpha}$ defined in (2.4) does not depend on $\alpha$, the norm in this space is depending on $\alpha$ as per the definition (2.3). Thus, for better understanding we keep the notation $\mathcal{H}_{\alpha}$ throughout the paper.

Also, the $\mathcal{H}_{\alpha}$-norm arises naturally in the regularity result in Proposition 2.3 and thus the constants appearing in (2.7)-(2.8) are independent on $\alpha$. We further observe that, for given $\alpha \geq 0$, the $\mathcal{H}_{\alpha}$ norm in (2.3) is equivalent to the $H^{1}$-norm, thanks to the Poincaré inequality.

Let us recall the coupling matrix $\mathcal{M}_{a}$ defined in (1.5) and we denote

$$
\mathcal{A}_{\alpha, a}=\mathcal{A}_{\alpha}+\mathcal{M}_{a} \text {, with the same domain } D\left(\mathcal{A}_{\alpha, a}\right):=D\left(\mathcal{A}_{\alpha}\right) .
$$

In particular, $\mathcal{A}_{\alpha, 0}:=\mathcal{A}_{\alpha}$.

By definition, it is clear that $\mathcal{A}_{\alpha, a}$ is no more self-adjoint for any $a \neq 0$ and more precisely, $\mathcal{A}_{\alpha, a}$ has been obtained by a bounded perturbation $\mathcal{M}_{a}$ to the self-adjoint operator $\mathcal{A}_{\alpha}$.

Proposition 2.2 (Existence of analytic semigroup) The operator $\left(-\mathcal{A}_{\alpha, a}, D\left(\mathcal{A}_{\alpha, a}\right)\right)$ defined by (2.5), generates an analytic semigroup in $E$.

Proof Let us first introduce the following densely defined bilinear form $h$; for all $u:=\left(u_{1}, u_{2}\right), \varphi:=\left(\varphi_{1}, \varphi_{2}\right) \in \mathcal{H}_{\alpha}$ (defined by (2.4)), we consider

$$
h(u, \varphi)=\sum_{i=1}^{2} \int_{0}^{1} \gamma_{i}(x) u_{i}^{\prime}(x) \varphi_{i}^{\prime}(x) d x+a \int_{0}^{1} u_{1}(x) \varphi_{2}(x) d x+\alpha u_{1}(1) \varphi_{1}(1) .
$$

It is clear that $h$ is continuous in $\mathcal{H}_{\alpha}$ with

$$
|h(u, \varphi)| \leq \kappa_{1}\|u\|_{\mathcal{H}_{\alpha}}\|\varphi\|_{\mathcal{H}_{\alpha}}, \quad \forall u, \varphi \in \mathcal{H}_{\alpha},
$$


where $\kappa_{1}>0$ depends on the diffusion coefficients $\gamma_{i}, i=1,2$, and the coupling coefficient $a$. On the other hand, we have

$$
h(u, u) \geq\|u\|_{\mathcal{H}_{\alpha}}^{2}-|a|\|u\|_{E}^{2}, \quad \forall u \in \mathcal{H}_{\alpha} .
$$

Then, by [40, Proposition 1.51 and Theorem 1.52], the negative of the operator associated with $h$ generates an analytic semigroup in $E$ of angle $\left(\pi / 2-\arctan \kappa_{1}\right)$. One can show that this operator is indeed $\mathcal{A}_{\alpha, a}$ with its domain $D\left(\mathcal{A}_{\alpha, a}\right)=D\left(\mathcal{A}_{\alpha}\right)$ (as defined in (2.5)). Henceforth, the proof is complete.

Proposition 2.3 (Regularity) Let $f=\left(f_{1}, f_{2}\right) \in L^{2}(0, T ; E)$ be any given source term.

1. For any given initial data $y_{0}=\left(y_{0,1}, y_{0,2}\right) \in E$, there exists a unique weak solution $y=\left(y_{1}, y_{2}\right) \in \mathcal{C}^{0}([0, T] ; E) \cap L^{2}\left(0, T ; \mathcal{H}_{\alpha}\right)$ satisfying the following energy estimate

$$
\begin{aligned}
\|y\|_{C^{0}([0, T] ; E)}+\|y\|_{L^{2}\left(0, T ; \mathcal{H}_{\alpha}\right)}+\left\|\partial_{t} y\right\|_{L^{2}\left(0, T ; \mathcal{H}_{-\alpha}\right)} \\
\\
\quad \leq C_{T, a}\left(\left\|y_{0}\right\|_{E}+\|f\|_{L^{2}(0, T ; E)}\right) .
\end{aligned}
$$

2. For any initial data $y_{0} \in \mathcal{H}_{\alpha}$, the weak solution y belongs to the space $\mathcal{C}^{0}\left([0, T] ; \mathcal{H}_{\alpha}\right) \cap$ $L^{2}\left(0, T ;\left(H^{2}(0,1)\right)^{2}\right)$ and satisfies

$$
\begin{aligned}
\|y\|_{L^{\infty}\left(0, T ; \mathcal{H}_{\alpha}\right)}+\|y\|_{L^{2}\left(0, T ;\left(H^{2}(0,1)\right)^{2}\right)} & +\left\|\partial_{t} y\right\|_{L^{2}(0, T ; E)} \\
& \leq C_{T, a}\left(\left\|y_{0}\right\|_{\mathcal{H}_{\alpha}}+\|f\|_{L^{2}(0, T ; E)}\right) .
\end{aligned}
$$

The proof of the above proposition can be drawn in a standard fashion, see for instance [36, Theorem 1.1, Chapter 4] or [24, Chapter 7]. We omit the details here.

2.2 The system with non-homogeneous Dirichlet data

We consider here a similar coupled system as previous but with some non-smooth Dirichlet boundary data; the system under study is the following

$$
\begin{cases}\partial_{t} y_{1}-\partial_{x}\left(\gamma_{1} \partial_{x} y_{1}\right)=f_{1} & \text { in }(0, T) \times(0,1), \\ \partial_{t} y_{2}-\partial_{x}\left(\gamma_{2} \partial_{x} y_{2}\right)+a y_{1}=f_{2} & \text { in }(0, T) \times(0,1), \\ y_{1}(t, 0)=g_{1} & \text { in }(0, T), \\ y_{2}(t, 0)=g_{2} & \text { in }(0, T), \\ y_{1}(t, 1)=y_{2}(t, 1) & \text { in }(0, T), \\ \gamma_{1}(1) \partial_{x} y_{1}(t, 1)+\gamma_{2}(1) \partial_{x} y_{2}(t, 1)+\alpha y_{1}(t, 1)=0 & \text { in }(0, T), \\ y_{1}(0, \cdot)=y_{0,1}(\cdot), \quad y_{2}(0, \cdot)=y_{0,2}(\cdot) & \text { in }(0,1) .\end{cases}
$$

We hereby introduce the adjoint of the operator $\mathcal{A}_{\alpha, a}$ (given by (2.5)) as follows:

$$
\mathcal{A}_{\alpha, a}^{*}=\mathcal{A}_{\alpha}^{*}+\mathcal{M}_{a}^{*}=\left(\begin{array}{cc}
-\partial_{x}\left(\gamma_{1} \partial_{x}\right) & a \\
0 & -\partial_{x}\left(\gamma_{2} \partial_{x}\right)
\end{array}\right)
$$

with its domain $D\left(\mathcal{A}_{\alpha, a}^{*}\right)=D\left(\mathcal{A}_{\alpha, a}\right)=D\left(\mathcal{A}_{\alpha}\right)$ (see $\left.(2.2 \mathrm{~b})\right)$. 
Remark 2.4 We could have replaced $\mathcal{A}_{\alpha}^{*}$ simply by $\mathcal{A}_{\alpha}$ as this operator is selfadjoint, but in order to be consistent with the non self-adjoint case (that is when $a \neq 0$ ), we decide to keep the notation $\mathcal{A}_{\alpha}^{*}$ in several places.

Observe that the operator $-\mathcal{A}_{\alpha, a}^{*}$ also generates an analytic semigroup in $E$, thanks to the Proposition 2.2 and we denote this semigroup by $\left(e^{-t \mathcal{A}_{\alpha, a}^{*}}\right)_{t>0}$. Indeed, the solution to the adjoint system of (2.1), for any given $\zeta \in \mathcal{H}_{\alpha}$, satisfies the regularity result proved in point 2 of Proposition 2.3. Using this, one can classically obtain the well-posedness of the solution to (2.9) in a dual sense as in $[20,41]$.

Proposition 2.5 For any $y_{0}:=\left(y_{0,1}, y_{0,2}\right) \in \mathcal{H}_{-\alpha}, f:=\left(f_{1}, f_{2}\right) \in L^{2}(0, T ; E)$ and $g:=\left(g_{1}, g_{2}\right) \in L^{2}\left(0, T ; \mathbb{R}^{2}\right)$, there exists a unique $y \in \mathcal{C}^{0}\left([0, T] ; \mathcal{H}_{-\alpha}\right) \cap L^{2}(0, T ; E)$, solution to (2.9), in the following sense: for any $t \in[0, T]$ and $\zeta:=\left(\zeta_{1}, \zeta_{2}\right) \in \mathcal{H}_{\alpha}$, we have

$$
\begin{aligned}
\langle y(t), \zeta\rangle_{\mathcal{H}_{-\alpha}, \mathcal{H}_{\alpha}}=\left\langle y_{0}, e^{\left.-t \mathcal{A}_{\alpha, a}^{*} \zeta\right\rangle_{\mathcal{H}_{-\alpha}}, \mathcal{H}_{\alpha}}+\int_{0}^{t}\left(f(s), e^{\left.-(t-s) \mathcal{A}_{\alpha, a}^{*} \zeta\right)}\right)_{E} d s\right. \\
-\int_{0}^{t}\left\langle g(s),\left.\left(\frac{\partial}{\partial \nu_{\gamma}}\left(e^{-(t-s) \mathcal{A}_{\alpha, a}^{*} \zeta}\right)(x)\right)\right|_{x=0}\right\rangle_{\mathbb{R}^{2}} d s .
\end{aligned}
$$

2.3 Formulation of control problems

We shall now formulate the null-control problems in terms of the following proposition.

Proposition 2.6 Let $y_{0} \in \mathcal{H}_{-\alpha}, a \in \mathbb{R}, \alpha \geq 0$ and any finite time $T>0$ be given. Also recall the set $U$ as defined in Proposition 2.5.

1. A function $v \in L^{2}(0, T ; \mathbb{R})$ is a null-control for the problem (1.7)-(1.8a), if and only if it satisfies: for any $\zeta \in \mathcal{H}_{\alpha}$

$$
\begin{aligned}
& -\left\langle y_{0}, e^{\left.-t \mathcal{A}_{\alpha, a}^{*} \zeta\right\rangle_{\mathcal{H}_{-\alpha}}, \mathcal{H}_{\alpha}}\right. \\
& \quad=\gamma_{2}(0) \int_{0}^{T} v(t)\left\langle\left(\begin{array}{l}
0 \\
1
\end{array}\right),\left.\left(\partial_{x}\left(e^{-(T-t) \mathcal{A}_{\alpha, a}^{*} \zeta}\right)(x)\right)\right|_{x=0}\right\rangle_{\mathbb{R}^{2}} d t .
\end{aligned}
$$

2. A function $v \in L^{2}(0, T)$ is a null-control for the problem (1.7)-(1.8b), if and only if it satisfies: for any $\zeta \in \mathcal{H}_{\alpha}$

$$
\begin{aligned}
& -\left\langle y_{0}, e^{\left.-t \mathcal{A}_{\alpha, a}^{*} \zeta\right\rangle_{\mathcal{H}_{-\alpha}, \mathcal{H}_{\alpha}}}\right. \\
& \quad=\gamma_{1}(0) \int_{0}^{T} v(t)\left\langle\left(\begin{array}{l}
1 \\
0
\end{array}\right),\left.\left(\partial_{x}\left(e^{-(T-t) \mathcal{A}_{\alpha, a}^{*} \zeta}\right)(x)\right)\right|_{x=0}\right\rangle_{\mathbb{R}^{2}} d t .
\end{aligned}
$$

It is hereby convenient to define the observation operators (independent on the quantities $a$ or $\alpha$ ) associated with (1.7)-(1.8a) and (1.7)-(1.8b) resp. as follows

$$
\begin{aligned}
& \mathcal{B}_{1}^{*}: u=\left(u_{1}, u_{2}\right) \in\left(H^{2}(0,1)\right)^{2} \mapsto \gamma_{2}(0) u_{2}^{\prime}(0), \\
& \mathcal{B}_{2}^{*}: u=\left(u_{1}, u_{2}\right) \in\left(H^{2}(0,1)\right)^{2} \mapsto \gamma_{1}(0) u_{1}^{\prime}(0) .
\end{aligned}
$$

In the next two sections, we develop the required results to prove the main Theorems 1.2 and 1.3. 
3 Boundary controllability of the system with control on the second component

This section is devoted to prove the existence of a null-control of the coupled system (1.7)-(1.8a), in terms of finding a proper observability inequality, and so a Carleman-type estimate is the primal ingredient to obtain. We hereby remind that the diffusion coefficients $\gamma_{i}, i=1,2$ satisfy the property (1.3).

\subsection{A global boundary Carleman estimate}

Let us first write the adjoint system to (1.7)-(1.8a), with the homogeneous Dirichlet conditions at the left end point.

$$
\begin{cases}-\partial_{t} q_{1}-\partial_{x}\left(\gamma_{1} \partial_{x} q_{1}\right)+a q_{2}=0 & \text { in }(0, T) \times(0,1), \\ -\partial_{t} q_{2}-\partial_{x}\left(\gamma_{2} \partial_{x} q_{2}\right)=0 & \text { in }(0, T) \times(0,1), \\ q_{1}(t, 0)=q_{2}(t, 0)=0 & \text { in }(0, T), \\ q_{1}(t, 1)=q_{2}(t, 1) & \text { in }(0, T), \\ \gamma_{1}(1) \partial_{x} q_{1}(t, 1)+\gamma_{2}(1) \partial_{x} q_{2}(t, 1)+\alpha q_{1}(t, 1)=0 & \text { in }(0, T), \\ q_{1}(T, \cdot)=\zeta_{1}(\cdot), q_{2}(T, \cdot)=\zeta_{2}(\cdot) & \text { in }(0,1),\end{cases}
$$

where the regularity of $\zeta:=\left(\zeta_{1}, \zeta_{2}\right)$ will be imposed later when needed and for simplicity sometimes we shall use the notation $Q:=(0, T) \times(0,1)$.

Now, we introduce the following space

$$
\begin{array}{r}
\mathcal{Q}:=\left\{q=\left(q_{1}, q_{2}\right) \in\left(\mathcal{C}^{2}(\bar{Q})\right)^{2} \mid q_{1}(t, 0)=q_{2}(t, 0)=0, q_{1}(t, 1)=q_{2}(t, 1),\right. \\
\left.\sum_{i=1}^{2} \gamma_{i}(1) \partial_{x} q_{i}(t, 1)+\alpha q_{1}(t, 1)=0, \forall t \in(0, T)\right\} .
\end{array}
$$

Before introducing the main theorem regarding Carleman estimate, we define some standard weight functions which are the key elements to obtain the Carleman inequality. In what follows, we follow the classical methodology for proving Carleman estimates introduced in [29] (see also [34]).

Construction of the weight functions. We consider the following affine functions

$$
\left\{\begin{array}{l}
\beta_{i}(x)=2+c_{i}(x-1), \quad \forall x \in[0,1] \\
\text { with } c_{1}=1, \quad c_{2}:=c_{2}\left(\gamma_{1}, \gamma_{2}\right)<0
\end{array}\right.
$$

which have the following properties

$$
\beta_{2} \geq \beta_{1}>0, \text { in }[0,1], \quad \beta_{2}(1)=\beta_{1}(1),
$$

and that constant $c_{2}<0$ (depending only on $\gamma_{1}, \gamma_{2}$ ) is chosen in such a way that satisfies

$$
\left|c_{2}\right| \geq 4 \quad \text { and } \quad c_{2}^{2} \gamma_{2}^{2}(1)\left(\left|c_{2}\right|-6\right)-7 \gamma_{1}^{2}(1) \geq 1
$$


Remark 3.1 We will see while proving a Carleman estimate (namely the Theorem 3.2 stated later), that the above assumption (3.4) is very sharp and crucial to absorb some unusual boundary integrals sitting in the right hand side of the Carleman estimate.

Now, we assume that $\lambda>1$ and $K=2 \max \left\{\left\|\beta_{1}\right\|_{\infty},\left\|\beta_{2}\right\|_{\infty}\right\}$ and define the weight functions $\varphi_{i}$ and $\eta_{i}$, for $i=1,2$, as follows

$$
\varphi_{i}(t, x)=\frac{e^{\lambda \beta_{i}(x)}}{t(T-t)}, \quad \eta_{i}(t, x)=\frac{e^{\lambda K}-e^{\lambda \beta_{i}(x)}}{t(T-t)}, \quad \forall(t, x) \in Q .
$$

From the properties of $\beta_{1}$ and $\beta_{2}$ in (3.3), we have that the functions $\varphi_{i}$ and $\eta_{i}$ are positive and satisfy

$$
\varphi_{1}(t, 1)=\varphi_{2}(t, 1) \text { and } \eta_{1}(t, 1)=\eta_{2}(t, 1)
$$

since $\beta_{1}(1)=\beta_{2}(1)$.

We also have the following relations in $Q$, for $i=1,2$,

$$
\left\{\begin{array}{l}
\partial_{x} \varphi_{i}=\lambda \varphi_{i} c_{i}, \quad \partial_{x} \eta_{i}=-\lambda \varphi_{i} c_{i} \\
\partial_{t} \varphi_{i}=\varphi_{i} \frac{2 t-T}{t(T-t)}, \quad \partial_{t} \eta_{i}=\eta_{i} \frac{2 t-T}{t(T-t)} \\
\partial_{t}^{2} \eta_{i}=\eta_{i} \frac{3(2 t-T)^{2}+T^{2}}{2 t^{2}(T-t)^{2}}
\end{array}\right.
$$

Now, we write the main theorem of this section concerning the Carleman estimate.

Theorem 3.2 (A Carleman estimate) Let the weight functions $\varphi_{1}, \varphi_{2}$ and $\eta_{1}, \eta_{2}$ be defined as in (3.5). Then, there exists $\lambda_{1}:=\lambda_{1}\left(\gamma_{1}, \gamma_{2}, \alpha\right)>0, s_{1}:=\left(T^{2}+T\right) \sigma_{1}>0$ with some $\sigma_{1}:=\sigma_{1}\left(\gamma_{1}, \gamma_{2}, \alpha\right)>0$ and a constant $C^{\prime}:=C^{\prime}\left(\gamma_{1}, \gamma_{2}, \alpha\right)>0$, such that the following Carleman estimate holds true

$$
\begin{aligned}
s^{3} \lambda^{4} \sum_{i=1}^{2} & \int_{0}^{T} \int_{0}^{1} e^{-2 s \eta_{i}} \varphi_{i}^{3}\left|q_{i}\right|^{2} d x d t+s \lambda^{2} \sum_{i=1}^{2} \int_{0}^{T} \int_{0}^{1} e^{-2 s \eta_{i}} \varphi_{i}\left|\partial_{x} q_{i}\right|^{2} d x d t \\
& +s^{3} \lambda^{3} \int_{0}^{T} \varphi_{1}(t, 1) e^{-2 s \eta_{1}(t, 1)}\left|q_{1}(t, 1)\right|^{2} d t \\
\leq & C^{\prime}\left[\sum_{i=1}^{2} \int_{0}^{T} \int_{0}^{1} e^{-2 s \eta_{i}}\left|\partial_{t} q_{i}+\partial_{x}\left(\gamma_{i} \partial_{x} q_{i}\right)\right|^{2} d x d t\right. \\
& \left.+s \lambda \int_{0}^{T} \varphi_{2}(t, 0) e^{-2 s \eta_{2}(t, 0)}\left|\partial_{x} q_{2}(t, 0)\right|^{2} d t\right],
\end{aligned}
$$

for $s \geq s_{1}, \lambda \geq \lambda_{1}$ and for all $\left(q_{1}, q_{2}\right) \in \mathcal{Q}$.

Before going to the proof of the theorem above, we let any $s>0, \lambda>1$ and $\left(q_{1}, q_{2}\right) \in \mathcal{Q}$ and we write $f_{i}=\partial_{t} q_{i}+\partial_{x}\left(\gamma_{i} \partial_{x} q_{i}\right)$, then $f_{i} \in L^{2}(Q)$, for $i=1,2$. We also set

$$
\psi_{i}(t, x)=e^{-s \eta_{i}(t, x)} q_{i}(t, x), \quad \forall(t, x) \in Q, \text { for } i=1,2 .
$$

Observe that,

$$
\psi_{i}(t, 0)=0, i=1,2, \text { and } \psi_{1}(t, 1)=\psi_{2}(t, 1)
$$


using (3.6) and the properties of $q_{i}, i=1,2$ in $\mathcal{Q}$. Also look that

$$
\partial_{x} \psi_{i}(t, x)=e^{-s \eta_{i}(t, x)} \partial_{x} q_{i}(t, x)+s \lambda \beta_{i}^{\prime}(x) \varphi_{i}(t, x) \psi_{i}(t, x), \forall(t, x) \in Q,
$$

so that we have

$$
\sum_{i=1}^{2} \gamma_{i}(1) \partial_{x} \psi_{i}(t, 1)=-\alpha \psi_{1}(t, 1)+s \lambda\left(\sum_{i=1}^{2} c_{i} \gamma_{i}(1)\right) \varphi_{1}(t, 1) \psi_{1}(t, 1)
$$

thanks to the boundary condition $\sum_{i=1}^{2} \gamma_{i}(1) \partial_{x} q_{i}(t, 1)+\alpha q_{1}(t, 1)=0$, the properties of $\varphi_{i}$ in (3.6) and $\psi_{i}$ in (3.9), and the fact that $\beta_{i}^{\prime}=c_{i}$, for $i=1,2$.

Next, we see that the functions $\psi_{i}$ satisfies the following relations in $Q$

$$
M_{1} \psi_{i}+M_{2} \psi_{i}=F_{i}, \quad \text { for } i=1,2,
$$

with

$$
\left\{\begin{array}{l}
M_{1} \psi_{i}=\partial_{x}\left(\gamma_{i} \partial_{x} \psi_{i}\right)+s^{2} \lambda^{2} c_{i}^{2} \varphi_{i}^{2} \gamma_{i} \psi_{i}+s\left(\partial_{t} \eta_{i}\right) \psi_{i} \\
M_{2} \psi_{i}=\partial_{t} \psi_{i}-2 s \lambda c_{i} \varphi_{i}\left(\gamma_{i} \partial_{x} \psi_{i}\right)-2 s \lambda^{2} c_{i}^{2} \varphi_{i} \gamma_{i} \psi_{i} \\
F_{i}=e^{-s \eta_{i}} f_{i}+s \lambda c_{i} \gamma_{i}^{\prime} \varphi_{i} \psi_{i}-s \lambda^{2} c_{i}^{2} \varphi_{i} \gamma_{i} \psi_{i}
\end{array}\right.
$$

We have for $i=1,2$,

$$
\left\|M_{1} \psi_{i}\right\|_{L^{2}(Q)}^{2}+\left\|M_{2} \psi_{i}\right\|_{L^{2}(Q)}^{2}+2\left(M_{1} \psi_{i}, M_{2} \psi_{i}\right)_{L^{2}(Q)}=\left\|F_{i}\right\|_{L^{2}(Q)}^{2} .
$$

Now, we present the following auxiliary lemma which is important to prove the main result in Theorem 3.2.

Lemma 3.3 Let the functions $\varphi_{i}, \eta_{i}, \psi_{i}, M_{1} \psi_{i}, M_{2} \psi_{2}$ in $Q$, for $i=1,2$, and the quantities $c_{1}, c_{2}$ be as introduced earlier. Then there exists $\lambda_{0}:=\lambda_{0}\left(\gamma_{1}, \gamma_{2}\right)>0, s_{0}:=$ $\left(T^{2}+T\right) \sigma_{0}>0$ with some $\sigma_{0}:=\sigma_{0}\left(\gamma_{1}, \gamma_{2}\right)>0$ and a constant $C^{\prime \prime}=C^{\prime \prime}\left(\gamma_{1}, \gamma_{2}\right)>0$ 
such that we have the following inequality

$$
\begin{aligned}
& \frac{1}{2} \sum_{i=1}^{2}\left\|M_{1} \psi_{i}\right\|_{L^{2}(Q)}^{2}+\frac{1}{2} \sum_{i=1}^{2}\left\|M_{2} \psi_{i}\right\|_{L^{2}(Q)}^{2} \\
& \quad+\sum_{i=1}^{2} s^{3} \lambda^{4} \int_{0}^{T} \int_{0}^{1} \varphi_{i}^{3}\left|\psi_{i}\right|^{2} d x d t+\sum_{i=1}^{2} s \lambda^{2} \int_{0}^{T} \int_{0}^{1} \varphi_{i}\left|\partial_{x} \psi_{i}\right|^{2} d x d t \\
& \quad+\sum_{i=1}^{2} \gamma_{i}(1) \int_{0}^{T} \partial_{x} \psi_{i}(t, 1) \partial_{t} \psi_{i}(t, 1) d t-s \lambda \sum_{i=1}^{2} c_{i} \int_{0}^{T} \varphi_{i}(t, 1)\left|\gamma_{i}(1) \partial_{x} \psi_{i}(t, 1)\right|^{2} d t \\
& \quad+s \lambda \sum_{i=1}^{2} c_{i} \int_{0}^{T} \varphi_{i}(t, 0)\left|\gamma_{i}(0) \partial_{x} \psi_{i}(t, 0)\right|^{2} d t \\
& \quad-2 s \lambda^{2} \sum_{i=1}^{2} c_{i}^{2} \gamma_{i}^{2}(1) \int_{0}^{T} \varphi_{i}(t, 1) \psi_{i}(t, 1) \partial_{x} \psi_{i}(t, 1) d t \\
& \quad-s^{3} \lambda^{3} c_{i}^{3} \sum_{i=1}^{2} \gamma_{i}^{2}(1) \int_{0}^{T} \varphi_{i}^{3}(t, 1)\left|\psi_{i}(t, 1)\right|^{2} d t \\
& \quad-s^{2} \lambda \sum_{i=1}^{2} c_{i} \gamma_{i}(1) \int_{0}^{T} \varphi_{i}(t, 1)\left(\partial_{t} \eta_{i}\right)(t, 1)\left|\psi_{i}(t, 1)\right|^{2} d t \\
& \leq C^{\prime \prime} \sum_{i=1}^{2}\left\|e^{-s \eta_{i}} f_{i}\right\|_{L^{2}(Q)}^{2},
\end{aligned}
$$

for all $\lambda \geq \lambda_{0}, s \geq s_{0}$.

In this paper, we decide to omit the proof for this auxiliary result in Lemma 3.3 , as the computations can be done in a similar spirit as of the proof of $[27$, Lemma 1]. Indeed, a rigorous proof of Lemma 3.3 can be found in [11, Lemma IV.3.3].

We now focus on obtaining the Carleman estimate (3.8) which is the main concern of this section.

Proof (of Theorem 3.2)

The idea to prove this theorem is to play with the boundary integrals of the inequality (3.14), so that we can absorb the lower order integrals by some leading terms and then to observe the proper observation term which will be eventually shifted in the right hand side of the actual estimate.

We make use of the following notations: denote all the six boundary terms respectively by $J_{k}, 1 \leq k \leq 6$, by maintaining the same order as in (3.14).

Before going to the proof, we recall that the constant $c_{1}=1$ (slope of $\beta_{1}$ ) and $c_{2}$ (slope of $\beta_{2}$ ) depends only on the diffusion coefficients $\gamma_{1}, \gamma_{2}$. 
- We have (using $\psi_{2}(t, 1)=\psi_{1}(t, 1)$ )

$$
\begin{gathered}
J_{1}:=\int_{0}^{T}\left[\gamma_{1}(1) \partial_{x} \psi_{1}(t, 1)+\gamma_{2}(1) \partial_{x} \psi_{2}(t, 1)\right] \partial_{t} \psi_{1}(t, 1) d t \\
=-\alpha \int_{0}^{T} \psi_{1}(t, 1) \partial_{t} \psi_{1}(t, 1) d t+s \lambda\left(\sum_{i=1}^{2} c_{i} \gamma_{i}(1)\right) \int_{0}^{T} \varphi_{1}(t, 1) \psi_{1}(t, 1) \partial_{t} \psi_{1}(t, 1) d t \\
=-\frac{s \lambda}{2}\left(\sum_{i=1}^{2} c_{i} \gamma_{i}(1)\right) \int_{0}^{T}\left(\partial_{t} \varphi_{1}\right)(t, 1)\left|\psi_{1}(t, 1)\right|^{2} d t
\end{gathered}
$$

due to the condition (3.11) and the fact that $\psi_{1}(0, \cdot)=\psi_{1}(T, \cdot)=0$. Now, using $\left|\partial_{t} \varphi_{1}\right| \leq T \varphi_{1}^{2} \leq 2 T^{3} \varphi_{1}^{3}$, we obtain

$$
\left|J_{1}\right| \leq \widetilde{C} s \lambda T^{3} \int_{0}^{T} \varphi_{1}^{3}(t, 1)\left|\psi_{1}(t, 1)\right|^{2} d t
$$

for some constant $\widetilde{C}>0$, that depends on $T, \alpha, \gamma_{1}, \gamma_{2}$.

- Next, we write the second boundary term of (3.14) as $J_{2}:=J_{21}+J_{22}$, where we keep the second integral in the left hand side of (3.14) since

$$
J_{22}:=-s \lambda c_{2} \int_{0}^{T} \varphi_{2}(t, 1)\left|\gamma_{2}(1) \partial_{x} \psi_{2}(t, 1)\right|^{2} d t \geq 0
$$

due to the fact that $c_{2}<0$. Later, we will see that the integral $J_{22}$ will be used to absorb some lower order terms.

On the other hand, the first integral of the second boundary term $J_{2}$ is

$$
J_{21}:=-s \lambda c_{1} \int_{0}^{T} \varphi_{1}(t, 1)\left|\gamma_{1}(1) \partial_{x} \psi_{1}(t, 1)\right|^{2} d t
$$

where $c_{1}>0$ and so $J_{21} \leq 0$. So, we need to absorb those integrals by some higher order terms in the left hand side. Let us recall (3.11) to express

$\gamma_{1}(1) \partial_{x} \psi_{1}(t, 1)=-\alpha \psi_{1}(t, 1)-\gamma_{2}(1) \partial_{x} \psi_{2}(t, 1)+s \lambda\left(\sum_{i=1}^{2} c_{i} \gamma_{i}(1)\right) \varphi_{1}(t, 1) \psi_{1}(t, 1)$,

so that we have the following,

$$
\begin{aligned}
& \left|J_{21}\right| \leq 3 s \lambda \alpha^{2} c_{1} \int_{0}^{T} \varphi_{1}(t, 1)\left|\psi_{1}(t, 1)\right|^{2} d t+3 s \lambda c_{1} \int_{0}^{T} \varphi_{1}(t, 1)\left|\gamma_{2}(1) \partial_{x} \psi_{2}(t, 1)\right|^{2} d t \\
& +6 s^{3} \lambda^{3} c_{1}\left(\sum_{i=1}^{2} c_{i}^{2} \gamma_{i}^{2}(1)\right) \int_{0}^{T} \varphi_{1}^{3}(t, 1)\left|\psi_{1}(t, 1)\right|^{2} d t:=J_{21}^{1}+J_{21}^{2}+J_{21}^{3},
\end{aligned}
$$

with a simple observation (since $\left.\varphi_{1} \leq 4 T^{4} \varphi_{1}^{3}\right)$,

$$
J_{21}^{1} \leq \widetilde{C} s \lambda \alpha^{2} T^{4} \int_{0}^{T} \varphi_{1}^{3}(t, 1)\left|\psi_{1}(t, 1)\right|^{2} d t
$$


- Now, we look into the third boundary term, $J_{3}:=J_{31}+J_{32}$, where we have

$$
J_{31}:=s \lambda c_{1} \int_{0}^{T} \varphi_{1}(t, 0)\left|\gamma_{1}(0) \partial_{x} \psi_{1}(t, 0)\right|^{2} d t \geq 0
$$

since $c_{1}>0$ and the function $\varphi_{1}>0$, and so one can discard this term from the left hand side of (3.14).

On the other hand, we have

$$
\begin{aligned}
\left|J_{32}\right| & :=\left.s \lambda\left|c_{2} \int_{0}^{T} \varphi_{2}(t, 0)\right| \gamma_{2}(0) \partial_{x} \psi_{2}(t, 0)\right|^{2} d t \mid \\
& \leq \widetilde{C} s \lambda \int_{0}^{T} \varphi_{2}(t, 0) e^{-2 s \eta_{2}(t, 0)}\left|\partial_{x} q_{2}(t, 0)\right|^{2} d t
\end{aligned}
$$

following the expression of $\partial_{x} \psi_{2}$ given by (3.10) and using the fact that $\psi_{2}(t, 0)=$ 0 .

- Thereafter, we write the fourth boundary integral of (3.14) by $J_{4}:=J_{41}+J_{42}$, and we obtain the following

$$
\begin{aligned}
& \left|J_{41}\right|=2 s \lambda^{2} c_{1}^{2} \gamma_{1}^{2}(1) \int_{0}^{T} \varphi_{1}(t, 1)\left|\psi_{1}(t, 1) \partial_{x} \psi_{1}(t, 1)\right| d t \\
& \leq \widetilde{C} \epsilon s \lambda \int_{0}^{T} \varphi_{1}(t, 1)\left|\gamma_{1}(1) \partial_{x} \psi_{1}(t, 1)\right|^{2} d t+\frac{\widetilde{C}}{\epsilon} s \lambda^{3} T^{4} \int_{0}^{T} \varphi_{1}^{3}(t, 1)\left|\psi_{1}(t, 1)\right|^{2} d t
\end{aligned}
$$

where we have used the Young's inequality and the fact $\varphi_{1} \leq 2 T^{4} \varphi_{1}^{3}$. Now, for the first integral in the right hand side of (3.20), we use the estimate for $J_{21}$ given by (3.17) to obtain

$$
\begin{aligned}
\left|J_{41}\right| \leq \widetilde{C} \epsilon & s \lambda \int_{0}^{T} \varphi_{1}(t, 1)\left|\gamma_{2}(1) \partial_{x} \psi_{2}(t, 1)\right|^{2} d t \\
& +\widetilde{C}\left(\epsilon s^{3} \lambda^{3}+\epsilon s \lambda \alpha^{2} T^{4}+\frac{1}{\epsilon} s \lambda^{3} T^{4}\right) \int_{0}^{T} \varphi_{1}^{3}(t, 1)\left|\psi_{1}(t, 1)\right|^{2} d t .
\end{aligned}
$$

On the other hand, a similar computation as in (3.20) gives that

$$
\left|J_{42}\right| \leq \widetilde{C} \epsilon s \lambda \int_{0}^{T} \varphi_{2}(t, 1)\left|\gamma_{2}(1) \partial_{x} \psi_{2}(t, 1)\right|^{2} d t+\frac{\widetilde{C}}{\epsilon} s \lambda^{3} T^{4} \int_{0}^{T} \varphi_{2}^{3}(t, 1)\left|\psi_{2}(t, 1)\right|^{2} d t .
$$

- The fifth and the leading boundary term in the left hand side of (3.14) is

$$
J_{5}=s^{3} \lambda^{3}\left(-c_{2}^{3} \gamma_{2}^{2}(1)-c_{1}^{3} \gamma_{1}^{2}(1)\right) \int_{0}^{T} \varphi_{1}^{3}(t, 1)\left|\psi_{1}(t, 1)\right|^{2} d t
$$

by writing $\varphi_{2}(t, 1)=\varphi_{1}(t, 1)$.

- Finally, the sixth boundary term $J_{6}$ satisfies

$$
\left|J_{6}\right| \leq \widetilde{C} s^{2} \lambda T \int_{0}^{T} \varphi_{1}^{3}(t, 1)\left|\psi_{1}(t, 1)\right|^{2} d t
$$

due to the facts that $\varphi_{2}(t, 1)=\varphi_{1}(t, 1), \partial_{t} \eta_{2}(t, 1)=\partial_{t} \eta_{1}(t, 1)$ and $\left|\partial_{t} \eta_{1}\right| \leq T \varphi_{1}^{2}$. 
- Let us first try to show that the coefficient of the integral $\int_{0}^{T} \varphi_{1}^{3}(t, 1)\left|\psi_{1}(t, 1)\right|^{2} d t$ is positive in the left hand side of the main inequality (3.14). To deduce this, recall the quantities $J_{5}$ from (3.23) and $J_{21}^{3}$ from (3.17) and take those quantities in the left hand side of the main inequality (3.14), we see

$$
J_{5}-J_{21}^{3}=K_{1} s^{3} \lambda^{3} \int_{0}^{T} \varphi_{1}^{3}(t, 1)\left|\psi_{1}(t, 1)\right|^{2} d t
$$

with

$$
K_{1}=c_{2}^{2} \gamma_{2}^{2}(1)\left(\left|c_{2}\right|-6 c_{1}\right)-7 c_{1}^{3} \gamma_{1}^{2}(1) \geq 1
$$

thanks to the choices of $c_{1}, c_{2}$; see (3.2)-(3.4).

- Next, we recall $J_{22}$ and $J_{21}^{2}$ respectively given by (3.16) and (3.17), use $\varphi_{1}(t, 1)=$ $\varphi_{2}(t, 1)$, and we write the other leading boundary integral in the left hand side as follows

$$
J_{22}-J_{21}^{2}=K_{2} s \lambda \int_{0}^{T} \varphi_{2}(t, 1)\left|\gamma_{2}(1) \partial_{x} \psi_{2}(t, 1)\right|^{2} d t
$$

with $K_{2}=\left(\left|c_{2}\right|-3 c_{1}\right)=\left(\left|c_{2}\right|-3\right) \geq 1$, again thanks to the choices of $c_{1}, c_{2}$ in $(3.2)-(3.4)$.

- Now, we gather the leading boundary terms $J_{5}-J_{21}^{3}$ and $J_{22}-J_{21}^{2}$ given by (3.25) and (3.26) respectively, in the left hand side of our main inequality (3.14), and in the right hand side we consider all the estimates of the lower order terms namely, $J_{1}$ from (3.15), $J_{21}^{1}$ from (3.18), $J_{31}$ from (3.19), $J_{41}$ from (3.21), $J_{42}$ from (3.22) and $J_{6}$ from (3.24), so that the inequality (3.14) follows

$$
\begin{aligned}
& s^{3} \lambda^{4} \sum_{i=1}^{2} \int_{0}^{T} \int_{0}^{1} \varphi_{i}^{3}\left|\psi_{i}\right|^{2} d x d t++s \lambda^{2} \sum_{i=1}^{2} \int_{0}^{T} \int_{0}^{1} \varphi_{i}\left|\partial_{x} \psi_{i}\right|^{2} d x d t \\
& +K_{1} s^{3} \lambda^{3} \int_{0}^{T} \varphi_{1}^{3}(t, 1)\left|\psi_{1}(t, 1)\right|^{2} d t+K_{2} s \lambda \int_{0}^{T} \varphi_{2}(t, 1)\left|\gamma_{2}(1) \partial_{x} \psi_{2}(t, 1)\right|^{2} d t \\
& \leq \widetilde{C}\left[\sum_{i=1}^{2} \int_{0}^{T} \int_{0}^{1} e^{-2 s \eta_{i}}\left|f_{i}\right|^{2} d x d t+s \lambda \int_{0}^{T} \varphi_{2}(t, 0) e^{-2 s \eta_{2}(t, 0)}\left|\partial_{x} q_{2}(t, 0)\right|^{2} d t\right] \\
& +\widetilde{C} \epsilon s^{3} \lambda^{3} \int_{0}^{T} \varphi_{1}^{3}(t, 1)\left|\psi_{1}(t, 1)\right|^{2} d t+2 \widetilde{C} \epsilon s \lambda \int_{0}^{T} \varphi_{2}(t, 1)\left|\gamma_{2}(1) \partial_{x} \psi_{2}(t, 1)\right|^{2} d t \\
& +\left(\frac{2 \widetilde{C}}{\epsilon} s \lambda^{3} T^{4}+\widetilde{C}(1+\epsilon) s \lambda \alpha^{2} T^{4}+\widetilde{C} s^{2} \lambda T+\widetilde{C} s \lambda T^{3}\right) \int_{0}^{T} \varphi_{1}^{3}(t, 1)\left|\psi_{1}(t, 1)\right|^{2} d t
\end{aligned}
$$

By fixing $\epsilon:=\min \left\{\frac{K_{1}}{2 \widetilde{C}}, \frac{K_{2}}{4 \widetilde{C}}\right\}>0$, taking $\lambda \geq \lambda_{1}:=\lambda_{1}\left(\gamma_{1}, \gamma_{2}, \alpha\right)$ and $s \geq$ $s_{1}:=\left(T^{2}+T\right) \sigma_{1}\left(\gamma_{1}, \gamma_{2}, \alpha\right)>0$, where $\lambda_{1}$ and $\sigma_{1}$ are large enough so that all the boundary integrals, except the observation term, in right hand side can be absorbed by the corresponding integrals in left hand side of (3.27), which leads 
us

$$
\begin{array}{r}
s^{3} \lambda^{4} \sum_{i=1}^{2} \int_{0}^{T} \int_{0}^{1} \varphi_{i}^{3}\left|\psi_{i}\right|^{2} d x d t+s \lambda^{2} \sum_{i=1}^{2} \int_{0}^{T} \int_{0}^{1} \varphi_{i}\left|\partial_{x} \psi_{i}\right|^{2} d x d t \\
+s^{3} \lambda^{3} \int_{0}^{T} \varphi_{1}(t, 1)\left|\psi_{1}(t, 1)\right|^{2} d t \leq C^{\prime}\left[\sum_{i=1}^{2} \int_{0}^{T} \int_{0}^{1} e^{-2 s \eta_{i}}\left|f_{i}\right|^{2} d x d t\right. \\
\left.+s \lambda \int_{0}^{T} \varphi_{2}(t, 0) e^{-2 s \eta_{2}(t, 0)}\left|\partial_{x} q_{2}(t, 0)\right|^{2} d t\right],
\end{array}
$$

for all $\lambda \geq \lambda_{1}, s \geq s_{1}$ with the constant $C^{\prime}:=C^{\prime}\left(\gamma_{1}, \gamma_{2}, \alpha\right)$. Now, we recall the expression of $\partial_{x} \psi_{i}$ from (3.10), so that we have

$$
e^{-2 s \eta_{i}}\left|\partial_{x} q_{i}\right|^{2} \leq 2\left|\partial_{x} \psi_{i}\right|^{2}+2 s^{2} \lambda^{2}\left(c_{i}\right)^{2} \varphi_{i}^{2}\left|\psi_{i}\right|^{2}, \text { for } i=1,2,
$$

and that implies

$$
\begin{aligned}
& s \lambda^{2} \sum_{i=1}^{2} \int_{0}^{T} \int_{0}^{1} e^{-2 s \eta_{i}} \varphi_{i}\left|\partial_{x} q_{i}\right|^{2} d x d t \\
& \leq \widetilde{C} s \lambda^{2} \sum_{i=1}^{2} \int_{0}^{T} \int_{0}^{1} \varphi_{i}\left|\partial_{x} \psi_{i}\right|^{2} d x d t+\widetilde{C} s^{3} \lambda^{4} \sum_{i=1}^{2} \int_{0}^{T} \int_{0}^{1} \varphi_{i}^{3}\left|\psi_{i}\right|^{2} d x d t
\end{aligned}
$$

Finally, combining (3.28) and (3.29), and replacing $f_{i}=\partial_{t} q_{i}+\partial_{x}\left(\gamma_{i} \partial_{x} q_{i}\right)$, $i=1,2$, we obtain the required Carleman inequality (3.8).

3.2 Null-controllability in terms of a boundary observability inequality

The Carleman estimate indeed leads us to obtain the following observability inequality which is in fact a necessary and sufficient condition for null-controllability.

Proposition 3.4 (Observability inequality) For any $\zeta:=\left(\zeta_{1}, \zeta_{2}\right) \in \mathcal{H}_{\alpha}$, the associated solution $q:=\left(q_{1}, q_{2}\right) \in \mathcal{C}^{0}\left([0, T] ; \mathcal{H}_{\alpha}\right) \cap L^{2}\left(0, T ;\left(H^{2}(0,1)\right)^{2}\right)$ to $(3.1)$ satisfies the following observation estimate

$$
\|q(0)\|_{\mathcal{H}_{\alpha}}^{2} \leq C e^{C / T} \int_{0}^{T}\left|\partial_{x} q_{2}(t, 0)\right|^{2} d t
$$

for some constant $C:=C\left(\gamma_{1}, \gamma_{2}, \alpha, a\right)>0$ that does not depend on $T>0$ and $\zeta$.

Proof We shall prove the required observability inequality for $0<T \leq 1$ to show the existence of a control in $(0, T)$ for the system $(1.7)-(1.8 \mathrm{a})$; this will not lose the generality since for any time $\widetilde{T}>1$, a continuation of a control in $(0,1)$ by 0 will still be a control in $(0, \widetilde{T})$. Let us now focus on the proof.

For any given $\zeta \in \mathcal{H}_{\alpha}$, one can apply the Carleman inequality given by Theorem 3.2 to the solution $q$ of $(3.1)$, with $\partial_{t} q_{1}+\partial_{x}\left(\gamma_{1} \partial_{x} q_{1}\right)=a q_{2}$ and $\partial_{t} q_{2}+\partial_{x}\left(\gamma_{2} \partial_{x} q_{2}\right)=0$ 
to deduce

$$
\begin{gathered}
s^{3} \lambda^{4} \sum_{i=1}^{2} \int_{0}^{T} \int_{0}^{1} e^{-2 s \eta_{i}} \varphi_{i}^{3}\left|q_{i}\right|^{2} d x d t+s \lambda^{2} \sum_{i=1}^{2} \int_{0}^{T} \int_{0}^{1} e^{-2 s \eta_{i}} \varphi_{i}\left|\partial_{x} q_{i}\right|^{2} d x d t \\
+s^{3} \lambda^{3} \int_{0}^{T} \varphi_{1}(t, 1) e^{-2 s \eta_{1}(t, 1)}\left|q_{1}(t, 1)\right|^{2} d t \leq C^{\prime}\left[\int_{0}^{T} \int_{0}^{1} e^{-2 s \eta_{1}}\left|a q_{2}\right|^{2} d x d t\right. \\
\left.+s \lambda \int_{0}^{T} \varphi_{2}(t, 0) e^{-2 s \eta_{2}(t, 0)}\left|\partial_{x} q_{2}(t, 0)\right|^{2} d t\right] .
\end{gathered}
$$

Now, we use $1 \leq 8 T^{6} \varphi_{2}^{3}$ to see the first term in right hand side of the above estimate as

$$
\begin{aligned}
\int_{0}^{T} \int_{0}^{1} e^{-2 s \eta_{1}}\left|a q_{2}\right|^{2} d x d t & \leq 8 a^{2} T^{6} \int_{0}^{T} \int_{0}^{1} \varphi_{2}^{3} e^{-2 s \eta_{1}}\left|q_{2}\right|^{2} d x d t \\
& \leq 8 a^{2} T^{6} \int_{0}^{T} \int_{0}^{1} \varphi_{2}^{3} e^{-2 s \eta_{2}}\left|q_{2}\right|^{2} d x d t=: \widetilde{X}
\end{aligned}
$$

since $\beta_{2} \geq \beta_{1}$ and so $\eta_{2} \leq \eta_{1}$ by construction (see (3.5)) which implies $e^{-2 s \eta_{1}} \leq$ $e^{-2 s \eta_{2}}$ for any $s>0$.

We see that $\widetilde{X}$ can be absorbed by the term $s^{3} \lambda^{4} \int_{0}^{T} \int_{0}^{1} e^{-2 s \eta_{2}} \varphi_{2}^{3}\left|q_{2}\right|^{2} d x d t$ in left hand side of the estimate (3.30) for some choice of $s \geq s_{1}=\left(T^{2}+T\right) \sigma_{1}$ in Theorem 3.2, possibly with some different $\sigma_{1}>0$, and also using the fact that $s^{3} \lambda^{3} \geq s \lambda^{2} \alpha$, for any $\lambda \geq \lambda_{1}$ (may be with some larger $\lambda_{1}:=\lambda_{1}\left(\gamma_{1}, \gamma_{2}, \alpha\right)$ ), we obtain

$$
\begin{array}{r}
s \lambda^{2} \sum_{i=1}^{2} \int_{0}^{T} \int_{0}^{1} e^{-2 s \eta_{i}} \varphi_{i}\left|\partial_{x} q_{i}\right|^{2} d x d t+s \lambda^{2} \alpha \int_{0}^{T} \varphi_{1}(t, 1) e^{-2 s \eta_{1}(t, 1)}\left|q_{1}(t, 1)\right|^{2} d t \\
\leq C s \lambda \int_{0}^{T} \varphi_{2}(t, 0) e^{-2 s \eta_{2}(t, 0)}\left|\partial_{x} q_{2}(t, 0)\right|^{2} d t, \quad
\end{array}
$$

with some constant $C>0$ that now depends on $\gamma_{1}, \gamma_{2}, \alpha$ and $a$. From (3.32), it follows after some classical computations as developed in [27, Proposition 2], that

$$
\int_{T / 4}^{3 T / 4}\|q(t)\|_{\mathcal{H}_{\alpha}}^{2} \leq C e^{C s / T^{2}} \int_{0}^{T}\left|\partial_{x} q_{2}(t, 0)\right|^{2} d t .
$$

Now, thanks to the regularity result in point 2 of Proposition 2.3 (which is also valid for the adjoint system (3.1) with source term $f=0)$, we have $\|q(0)\|_{\mathcal{H}_{\alpha}}^{2} \leq$ $C_{a}\|q(t)\|_{\mathcal{H}_{\alpha}}^{2}$ for any $0<t \leq T(\leq 1)$. Using this and by choosing $s=s_{1}=\left(T^{2}+\right.$ T) $\sigma_{1}>0$ the inequality (3.33) reduces to

$$
\|q(0)\|_{\mathcal{H}_{\alpha}}^{2} \leq C e^{C \sigma\left(T^{2}+T\right) / T^{2}} \int_{0}^{T}\left|\partial_{x} q_{2}(t, 0)\right|^{2} d t
$$

which gives the required inequality of our proposition with the constant $C>0$, independent on $T$ and $\zeta$. 
Proof (of Theorem 1.2)

Once we have the above observability inequality, then by the Hilbert Uniqueness Method, one can prove the existence of a boundary null-control $v \in L^{2}(0, T)$ for the problem (1.7)-(1.8a); see for instance [35] where this kind of idea has been described (indeed, such an argument has been used for instance in [28] and the corresponding observability inequality is in Section 1.3.4 of this reference).

The estimation of the control cost $C e^{C / T}$ follows from the sharp observability inequality in Proposition 3.4.

Remark 3.5 For the other system, that is for (1.7)-(1.8b), the observation term is $\mathcal{B}_{2}^{*} q(t)=\gamma_{1}(0) \partial_{x} q_{1}(t, 0)$, and so to obtain a good Carleman estimate one has to choose the functions $\beta_{i}, i=1,2$ where $c_{1}=: c_{1}\left(\gamma_{1}, \gamma_{2}\right)<0$ with large enough modulus and $c_{2}=1$ (which is opposite to the previous case), to construct the suitable weight functions.

In that case, a Carleman estimate similar to (3.8) still holds with the observation integral

$$
s \lambda \int_{0}^{T} \varphi_{1}(t, 0) e^{-2 s \eta_{1}(t, 0)}\left|\partial_{x} q_{1}(t, 0)\right|^{2} d t
$$

but we cannot hope for a good observability inequality with observation term $\gamma_{1}(0) \partial_{x} q_{1}(t, 0)$. The reason behind this is the following: in this case, we have $e^{-2 s \eta_{1}} \geq e^{-2 s \eta_{2}}$ ( since $\beta_{1} \geq \beta_{2}$ now and consequently $\eta_{1} \leq \eta_{2}$ ), which will prevent us from absorbing the source term $\int_{0}^{T} \int_{0}^{1} e^{-2 s \eta_{1}}\left|a q_{2}\right|^{2} d x d t(a \neq 0)$ appeared in the right-hand side, by the term $s^{3} \lambda^{4} \int_{0}^{T} \int_{0}^{1} e^{-2 s \eta_{2}} \varphi_{2}^{3}\left|q_{2}\right|^{2} d x d t$ in left-hand side.

The above remark tells us that the Carleman trick is no more applicable to prove the boundary null-controllability of the system (1.7)-(1.8b).

In fact, it is not really a technical issue since, as we previously mentioned, the controllability property of the system (1.7)-(1.8b) will depend on the values of the coupling coefficient $a$ and the boundary parameter $\alpha$. This will be investigated in the next section.

\section{Boundary controllability of our system with control on the first component}

This section is devoted to find a control for the prescribed problem (1.7)-(1.8b) with $\gamma_{1}=\gamma_{2}=1$, using the moments technique; and as we know, the key point to develop and solve the moments problem is to obtain sharp estimates on spectral elements of the adjoint to the associated elliptic operator.

4.1 Description of spectrum of the underlying elliptic operator

In this section, we investigate some important spectral properties of the elliptic operator $\mathcal{A}_{\alpha, a}^{*}$ having the formal expression in $(2.10)$ with $\gamma_{1}=\gamma_{2}=1$.

Remark 4.1 For $\gamma_{1}=\gamma_{2}=1$ also, we keep the same symbol $\mathcal{A}_{\alpha, a}$ and $\mathcal{A}_{\alpha, a}^{*}$ (for any $a \in \mathbb{R}, \alpha \geq 0$ ) to denote the elliptic operator and its adjoint respectively. 
Below, we present the eigenvalue problem $\mathcal{A}_{\alpha, a}^{*} u=\lambda u$, for $\lambda \in \mathbb{C}$, that is explicitly

$$
\begin{cases}-u_{1}^{\prime \prime}+a u_{2}=\lambda u_{1} & \text { in }(0,1), \\ -u_{2}^{\prime \prime}=\lambda u_{2} & \text { in }(0,1), \\ u_{1}(0)=0, u_{2}(0)=0, & \\ u_{1}(1)=u_{2}(1) & \\ u_{1}^{\prime}(1)+u_{2}^{\prime}(1)+\alpha u_{1}(1)=0 . & \end{cases}
$$

We recall from (2.10) that for $a \neq 0$, the operator $\mathcal{A}_{\alpha, a}^{*}$ is no more self-adjoint and here we develop the spectral analysis of this operator (more precisely of its complex version) using some perturbation argument of linear operators which we discuss in Section 4.1.2. That is the reason why, we first need to describe the spectrum of the self-adjoint operator $\mathcal{A}_{\alpha}^{*}$.

\subsubsection{Spectrum of the self-adjoint operator $\mathcal{A}_{\alpha}^{*}$}

We directly start with the eigenvalue problem $\mathcal{A}_{\alpha} u=\mathcal{A}_{\alpha}^{*} u=\lambda u, u \neq 0$, where one may assume that $\lambda$ is real since $\mathcal{A}_{\alpha}$ is self-adjoint,

$$
\begin{cases}-u_{1}^{\prime \prime}=\lambda u_{1} & \text { in }(0,1), \\ -u_{2}^{\prime \prime}=\lambda u_{2} & \text { in }(0,1), \\ u_{1}(0)=0, u_{2}(0)=0, & \\ u_{1}(1)=u_{2}(1) & \\ u_{1}^{\prime}(1)+u_{2}^{\prime}(1)+\alpha u_{1}(1)=0 . & \end{cases}
$$

Observe first that we necessarily have $\lambda>0$. Indeed, multiplying the first and second equations by $u_{1}$ and $u_{2}$ respectively, then upon an integration by parts and using the boundary conditions, one has

$$
\int_{0}^{1}\left(\left|u_{1}^{\prime}(x)\right|^{2}+\left|u_{2}^{\prime}(x)\right|^{2}\right) d x+\alpha\left|u_{1}(1)\right|^{2}=\lambda \int_{0}^{1}\left(\left|u_{1}(x)\right|^{2}+\left|u_{2}(x)\right|^{2}\right) d x,
$$

which certainly tells us that $\lambda>0$ since $\alpha \geq 0$. We shall set $\mu=\sqrt{\lambda}$.

Let us now solve (4.2). We start by observing that if $u_{1}=0$ then the equation for $u_{2}$ along with the boundary conditions gives $u_{2}=0$. Therefore, by using the boundary condition at $x=0$, we expect the solution to be of the form

$$
u_{1}(x)=C_{1} \sin (\mu x), u_{2}(x)=C_{2} \sin (\mu x), \quad \forall x \in[0,1],
$$

for some $C_{1}, C_{2} \in \mathbb{R}$. On the other hand, the conditions $u_{1}(1)=u_{2}(1)$ and $u_{1}^{\prime}(1)+$ $u_{2}^{\prime}(1)+\alpha u_{1}(1)=0$ respectively provide

$$
\begin{array}{r}
\left(C_{1}-C_{2}\right) \sin \mu=0 \quad \text { and } \\
\mu C_{1} \cos \mu+\mu C_{2} \cos \mu+\alpha C_{1} \sin \mu=0 .
\end{array}
$$

- First, when $\sin \mu \neq 0$, then $C_{1}=C_{2} \neq 0$ from (4.3a), so that from (4.3b) we end up with

$$
2 \mu \cos \mu+\alpha \sin \mu=0 .
$$


- If $\alpha=0$, then $\mu_{k, 1}^{0}:=(k+1 / 2) \pi$ for $k \geq 0$ are the positive roots of the above equation. A first family of eigenvalues of (4.2) is thus given by $\lambda_{k, 1}^{0}:=(k+1 / 2)^{2} \pi^{2}, k \geq 0$.

- If $\alpha>0$, we rewrite the equation (4.4) as

$$
g(\mu):=\tan \mu+\frac{2}{\alpha} \mu=0 .
$$

We calculate that $g^{\prime}(\mu)=\sec ^{2} \mu+2 / \alpha>0$, and so in particular, $g^{\prime}(\mu)>0$ in $((k+1 / 2) \pi,(k+3 / 2) \pi)$, for any $k \geq 0$. Beside this, we have

$$
\lim _{\mu \rightarrow((k+1 / 2) \pi)^{+}} g(\mu)=-\infty, \quad g((k+1) \pi)=\frac{2}{\alpha}(k+1) \pi>0 .
$$

So, there exists exactly one root of $g$ in $((k+1 / 2) \pi,(k+1) \pi)$, for each $k \geq 0$, and given $\alpha>0$. Let us denote the roots of $g$ by $\mu_{k, 1}^{\alpha}$ and the eigenvalues by $\lambda_{k, 1}^{\alpha}:=\left(\mu_{k, 1}^{\alpha}\right)^{2}$, for all $k \geq 0$ and given any $\alpha>0$.

Note that an associated set of normalized eigenfunctions is given by

$$
\Phi_{\lambda_{k, 1}^{\alpha}}(x):=\left(\begin{array}{c}
\sin \left(\mu_{k, 1}^{\alpha} x\right) \\
\sin \left(\mu_{k, 1}^{\alpha} x\right)
\end{array}\right) .
$$

- Assume now that $\sin \mu=0$ (see (4.3a)), from which we deduce that $\mu=(k+1) \pi$ for some $k \geq 0$. By (4.3b) we have $C_{1}=-C_{2}$. Now, to be consistent with the notation, we shall denote this second set of eigenvalue-eigenfunction pairs by $\left\{\lambda_{k, 2}^{\alpha}, \Phi_{\lambda_{k, 2}^{\alpha}}\right\}_{k \geq 0}$, even if they are not depending on $\alpha$, with $\lambda_{k, 2}^{\alpha}=(k+1)^{2} \pi^{2}$ and

$$
\Phi_{\lambda_{k, 2}^{\alpha}}(x):=\left(\begin{array}{c}
\sin (k+1) \pi x \\
-\sin (k+1) \pi x
\end{array}\right)
$$

Remark 4.2 Since the operator $\mathcal{A}_{\alpha}^{*}$ is self-adjoint, the family $\left\{\Phi_{\lambda_{k, 1}^{\alpha}}, \Phi_{\lambda_{k, 2}^{\alpha}}\right\}_{k \geq 0}$ indeed forms an orthonormal basis of $E$.

Remark 4.3 For any $\alpha \geq 0$, we deduce the following asymptotic formula of $\lambda_{k, 1}^{\alpha}$,

$$
\lambda_{k, 1}^{\alpha}=\left(k+\frac{1}{2}\right)^{2} \pi^{2}+\alpha+o_{\alpha}(1), \text { for } k \text { large, }
$$

To obtain the above asymptotic, we express $\mu_{k, 1}^{\alpha}=(k+1 / 2) \pi+\delta_{k, 1}^{\alpha}$ with $\delta_{k, 1}^{\alpha} \in$ $(0, \pi / 2)$ for $\alpha>0$. Then we see from $g\left(\mu_{k, 1}^{\alpha}\right)=0$ that

$$
\begin{array}{r}
\tan \left((k+1 / 2) \pi+\delta_{k, 1}^{\alpha}\right)+\frac{2}{\alpha}\left((k+1 / 2) \pi+\delta_{k, 1}^{\alpha}\right)=0, \\
\text { i.e., } \frac{\cos \delta_{k, 1}^{\alpha}}{\sin \delta_{k, 1}^{\alpha}}=\frac{2}{\alpha}\left((k+1 / 2) \pi+\delta_{k, 1}^{\alpha}\right),
\end{array}
$$

of which, the right hand side goes to $+\infty$ as $k \rightarrow+\infty$, and so, for any fixed $\alpha>0$, $\delta_{k, 1}^{\alpha} \rightarrow 0^{+}$. Consequently, $\sin \delta_{k, 1}^{\alpha} \sim \delta_{k, 1}^{\alpha}$ and $\cos \delta_{k, 1}^{\alpha} \sim 1$ for large $k$. So, by taking into account (4.7), we have

$$
\delta_{k, 1}^{\alpha} \sim \frac{\alpha}{2 k \pi}, \text { for } k \text { large. }
$$


Henceforth, we deduce that

$$
\begin{aligned}
\lambda_{k, 1}^{\alpha}-\left(k+\frac{1}{2}\right)^{2} \pi^{2} & =\left(\mu_{k, 1}^{\alpha}-\left(k+\frac{1}{2}\right) \pi\right)\left(\mu_{k, 1}^{\alpha}+\left(k+\frac{1}{2}\right) \pi\right) \\
& =\delta_{k, 1}^{\alpha}\left((2 k+1) \pi+\delta_{k, 1}^{\alpha}\right) \\
& \stackrel{k \rightarrow \infty}{\longrightarrow} .
\end{aligned}
$$

4.1.2 Spectrum of the main operator $\mathcal{A}_{\alpha, a}^{*}$

We begin with our main problem of interest, that is the system of odes (4.1). For our use, we first denote the set of all eigenvalues of $\mathcal{A}_{\alpha, a}^{*}$ by $\Lambda_{\alpha, a}$ for any $a \in \mathbb{R}$ and $\alpha \geq 0$.

Let us choose $a \in \mathbb{R}^{*}$ and $\alpha \geq 0$ and we pursue some detailed analysis step by step as follows.

Localization of the spectrum. We observe that

$$
\Lambda_{\alpha, a} \subset \bigcup_{\lambda \in \Lambda_{\alpha, 0}} D(\lambda, 2|a|),
$$

where $\Lambda_{\alpha, 0}$ is the set of all eigenvalues of the self-adjoint operator $\mathcal{A}_{\alpha}=\mathcal{A}_{\alpha, 0}$.

Indeed, if $\xi \in \mathbb{C}$ is such that $|\xi-\lambda| \geq 2|a|$ for any $\lambda \in \Lambda_{\alpha, 0}$, then in particular $\mathcal{A}_{\alpha, 0}^{*}-\xi I$ is invertible and satisfies the resolvent estimate

$$
\left\|\left(\mathcal{A}_{\alpha, 0}^{*}-\xi I\right)^{-1}\right\|=\sup _{\lambda \in \Lambda_{\alpha, 0}} \frac{1}{|\xi-\lambda|} \leq \frac{1}{2|a|} .
$$

It follows that

$$
\mathcal{A}_{\alpha, a}^{*}-\xi I=\mathcal{A}_{\alpha, 0}^{*}-\xi I+\mathcal{M}_{a}^{*}=\left(\mathcal{A}_{\alpha, 0}^{*}-\xi I\right)\left(I-\left(\mathcal{A}_{\alpha, 0}^{*}-\xi I\right)^{-1} \mathcal{M}_{a}^{*}\right),
$$

and thus $\xi$ lies in the resolvent set of $\mathcal{A}_{\alpha, a}^{*}$ since

$$
\left\|\left(\mathcal{A}_{\alpha, 0}^{*}-\xi I\right)^{-1} \mathcal{M}_{a}^{*}\right\| \leq\left\|\left(\mathcal{A}_{\alpha, 0}^{*}-\xi I\right)^{-1}\right\|\left\|\mathcal{M}_{a}^{*}\right\| \leq \frac{1}{2|a|}|a|<1 .
$$

In particular, $\mathcal{A}_{\alpha, a}^{*}$ has compact resolvent since the self-adjoint operator $\mathcal{A}_{\alpha, 0}$ has so (see for instance [31, IV-Theorem 3.17]), which ensures that the spectrum of $\mathcal{A}_{\alpha, a}$ is discrete.

Multiplicity. Observe now that all eigenvalues have geometric multiplicity 1 . Assume that it is not the case, then we can find one associated eigenfunction $u=$ $\left(u_{1}, u_{2}\right)$ such that $u_{1}(1)=0$. By the boundary condition at $x=1$, we also have $u_{2}(1)=0$. Note that $u_{2}^{\prime}(1) \neq 0$ since if it were not the case, we would have $u_{2}=0$ in $(0,1)$ and then $u_{1}=0$ in $(0,1)$ which is not possible.

From (4.1), we see that $u_{2}$ satisfies a second order ode with homogeneous Dirichlet boundary conditions from which we deduce that $\lambda=(k+1)^{2} \pi^{2}$ for some $k \geq 0$ and, up to a multiplicative constant, $u_{2}(x)=\sin (k+1) \pi x, \forall x \in[0,1]$. In particular, $\lambda$ is real. 
Now, we multiply the differential equation of $u_{1}$ by $u_{2}$ and integrate, i.e.,

$$
\int_{0}^{1}-u_{1}^{\prime \prime}(x) u_{2}(x) d x+a \int_{0}^{1}\left|u_{2}(x)\right|^{2} d x=\lambda \int_{0}^{1} u_{1}(x) u_{2}(x) d x .
$$

Performing integration by parts and $u_{i}(0)=u_{i}(1)=0$ (for $i=1,2$ ), we get

$$
\int_{0}^{1}-u_{2}^{\prime \prime}(x) u_{1}(x) d x+a \int_{0}^{1}\left|u_{2}(x)\right|^{2} d x=\lambda \int_{0}^{1} u_{1}(x) u_{2}(x) d x .
$$

Now, since $-u_{2}^{\prime \prime}=\lambda u_{2}$ in $(0,1)$, and $a \neq 0$, we deduce from the above equality that $u_{2}=0$ in $[0,1]$ which is not possible as discussed above. The claim is proved.

The case $\lambda=0$. We observe that $\lambda=0$ is an eigenvalue if and only if $a+3 \alpha+6=0$. Take $\lambda=0$ in (4.1). Then solving the set of odes along with the homogeneous boundary condition at $x=0$, one obtain $u_{2}(x)=c_{1} x$ and $u_{1}(x)=c_{1} \frac{a x^{3}}{6}+c_{2} x$. Now, thanks to the Kirchhoff boundary condition at $x=1$, we obtain $c_{2}=c_{1}\left(1-\frac{a}{6}\right)$ and

$$
c_{1}(a+3 \alpha+6)=0,
$$

which shows that $c_{1}=0$ (consequently, $c_{2}=0$ ) provided $a+3 \alpha+6 \neq 0$; in that case, $\lambda=0$ is not an eigenvalue of $\mathcal{A}_{\alpha, a}^{*}$.

But, as soon as we have $a+3 \alpha+6=0$ (then fix $c_{1}=1$ ), we see that $\lambda=0$ is an eigenvalue with the eigenfunction

$$
\Phi_{0}(x)=\left(\begin{array}{c}
\frac{a x^{3}}{6}+\left(1-\frac{a}{6}\right) x \\
x
\end{array}\right), \quad \forall x \in[0,1] .
$$

The case $\lambda \neq 0$. As we have seen above we cannot have $u_{2}=0$ in $(0,1)$. We take $\mu \in \mathbb{C}$ such that $\mu^{2}=\lambda$ and we observe that the solution of (4.1) is necessarily of the form

$$
\left\{\begin{array}{l}
u_{1}(x)=\frac{a K_{1} x}{2 i \mu}\left(e^{i \mu x}+e^{-i \mu x}\right)+K_{2}\left(e^{i \mu x}-e^{-i \mu x}\right), \\
u_{2}(x)=K_{1}\left(e^{i \mu x}-e^{-i \mu x}\right),
\end{array}\right.
$$

for some $K_{1}, K_{2} \in \mathbb{C}$. Thereafter, the two boundary conditions at $x=1$ provides us the following two equations

$$
\left\{\begin{array}{l}
K_{1}\left(a c_{\mu}-2 i \mu s_{\mu}\right)+K_{2}\left(2 i \mu s_{\mu}\right)=0 \text { and } \\
K_{1}\left(-2 \mu^{2} c_{\mu}+a i \mu s_{\mu}+a c_{\mu}+2 i \alpha \mu s_{\mu}\right)+K_{2}\left(-2 \mu^{2} c_{\mu}\right)=0,
\end{array}\right.
$$

where we introduced $s_{\mu}:=2 i \sin \mu$ and $c_{\mu}:=2 \cos \mu$. Now, for the existence of non-zero solution $\left(K_{1}, K_{2}\right)$ of the above system, the following condition should necessarily be satisfied:

$$
8 \mu^{2} i\left(s_{\mu} c_{\mu}\right)+2 \mu a\left(s_{\mu}^{2}-c_{\mu}^{2}\right)-2 a i\left(s_{\mu} c_{\mu}\right)+4 \alpha \mu s_{\mu}^{2}=0,
$$

which is actually the determinant of the coefficient matrix of system (4.11). Since we have assumed that $\mu \neq 0$, the condition above implies that $s_{\mu} \neq 0$.

Using the relations $s_{\mu} c_{\mu}=2 i \sin 2 \mu, s_{\mu}^{2}=-4 \sin ^{2} \mu$ and $s_{\mu}^{2}-c_{\mu}^{2}=-4$, the above equation simplifies as

$$
\left(4 \mu^{2}-a\right) \sin 2 \mu+2 a \mu+4 \alpha \mu \sin ^{2} \mu=0 .
$$


Now, from the first equation of (4.11), we have $K_{2}=K_{1}\left(1-\frac{a c_{\mu}}{2 i \mu s_{\mu}}\right)=K_{1}\left(1+\frac{a}{2 \mu} \frac{\cos \mu}{\sin \mu}\right)$. Next we fix $K_{1}=1 / 2 i$ and deduce from (4.10) that the eigenfunction associated with $\lambda=\mu^{2}$ is given by

$$
\Phi_{\lambda}(x):=\left(\begin{array}{c}
-\frac{a x}{2 \mu} \cos (\mu x)+\left(1+\frac{a}{2 \mu} \frac{\cos \mu}{\sin \mu}\right) \sin (\mu x) \\
\sin (\mu x)
\end{array}\right),
$$

as soon as $\mu \in \mathbb{C}$ satisfies (4.12).

Real solutions of the transcendental equation (4.12). We set

$$
f(\mu):=\left(4 \mu^{2}-a\right) \sin 2 \mu+2 a \mu+4 \alpha \mu \sin ^{2} \mu .
$$

Our goal is to prove the following lemma.

Lemma 4.4 Let $a \in \mathbb{R}^{*}$ and $\alpha \geq 0$. There exists some $k_{\alpha, a} \in \mathbb{N} \cup\{0\}$, and $C_{\alpha, a}>0$ such that for each $k \geq k_{\alpha, a}$, the function $f$ has:

- at least one real root, denoted by $\mu_{k, 1}^{\alpha, a}$, in the interval

$$
((k+1 / 4) \pi,(k+3 / 4) \pi),
$$

and that satisfies

$$
\mu_{k, 1}^{\alpha, a}=(k+1 / 2) \pi+\frac{2 \alpha+a}{4 k \pi}+o_{\alpha, a}(1 / k),
$$

- at least one real root, denoted by $\mu_{k, 2}^{\alpha, a}$, in the interval

$$
((k+3 / 4) \pi,(k+5 / 4) \pi),
$$

and that satisfies

$$
\mu_{k, 2}^{\alpha, a}=(k+1) \pi-\frac{a}{4 k \pi}+o_{\alpha, a}(1 / k) .
$$

Proof Let $\epsilon=\mp \pi / 4$. Then, we observe the following facts.

- A straightforward computation gives

$$
f((k+1 / 2) \pi+\epsilon) \sim-4 \sin (2 \epsilon) k^{2} \pi^{2}, \quad \text { for large } k .
$$

Hence, for $k$ large enough, $f((k+1 / 4) \pi)$ and $f((k+3 / 4) \pi)$ have different signs, which proves the existence of a root $\mu_{k, 1}^{\alpha, a} \in((k+1 / 4) \pi,(k+3 / 4) \pi)$.

Let $\delta_{k}:=\mu_{k, 1}^{\alpha, a}-(k+1 / 2) \pi \in(-\pi / 4, \pi / 4)$. The equation $f\left(\mu_{k, 1}^{\alpha, a}\right)=0$ gives

$$
\begin{aligned}
-\left(4\left((k+1 / 2) \pi+\delta_{k}\right)^{2}-a\right) \sin \left(2 \delta_{k}\right) & +2 a\left((k+1 / 2) \pi+\delta_{k}\right) \\
+ & 4 \alpha\left((k+1 / 2) \pi+\delta_{k}\right) \cos ^{2} \delta_{k}=0,
\end{aligned}
$$

and in particular we get for some $C_{\alpha, a}>0$,

$$
\left|\sin \left(2 \delta_{k}\right)\right| \leq \frac{C_{\alpha, a}}{k},
$$

which implies that $\delta_{k} \rightarrow 0$ as $k \rightarrow+\infty$ and coming back to (4.16), one can deduce that

$$
\delta_{k}=\frac{(2 \alpha+a)}{4 k \pi}+O_{\alpha, a}\left(1 / k^{3}\right) .
$$


- Similarly, we have

$$
f((k+1) \pi+\epsilon) \sim 4 \sin (2 \epsilon) k^{2} \pi^{2}, \quad \text { for large } k,
$$

and by the similar trick as previous we get the existence of a root $\mu_{k, 2}^{\alpha, a} \in$ $((k+3 / 4) \pi,(k+5 / 4) \pi)$. Setting now $\delta_{k}:=\mu_{k, 2}^{\alpha, a}-(k+1) \pi \in(-\pi / 4, \pi / 4)$, the equation $f\left(\mu_{k, 2}^{\alpha, a}\right)=0$ gives

$$
\begin{aligned}
\left(4\left((k+1) \pi+\delta_{k}\right)^{2}-a\right) \sin \left(2 \delta_{k}\right)+2 a & \left((k+1) \pi+\delta_{k}\right) \\
& +4 \alpha\left((k+1) \pi+\delta_{k}\right) \sin ^{2} \delta_{k}=0,
\end{aligned}
$$

again from which we first deduce that $\delta_{k}$ tends to 0 (using the similar argument as before) and then

$$
\delta_{k}=-\frac{a}{4 k \pi}+O_{\alpha, a}\left(1 / k^{3}\right) .
$$

Corollary 4.5 For any $k \geq k_{\alpha, a}$, the operator $\mathcal{A}_{\alpha, a}^{*}$ has exactly two real eigenvalues $\lambda_{k, 1}^{\alpha, a}$ and $\lambda_{k, 2}^{\alpha, a}$ that satisfy

$$
\begin{gathered}
\lambda_{k, 1}^{\alpha, a}=(k+1 / 2)^{2} \pi^{2}+(\alpha+a / 2)+o_{\alpha, a}(1), \\
\lambda_{k, 2}^{\alpha, a}=(k+1)^{2} \pi^{2}-a / 2+o_{\alpha, a}(1) .
\end{gathered}
$$

Moreover, for each $k \geq k_{\alpha, a}$ (possibly some larger $k_{\alpha, a}$ than earlier) and $i \in\{1,2\}$, $\lambda_{k, i}^{\alpha, a}$ is the unique eigenvalue of $\mathcal{A}_{\alpha, a}^{*}$ in the following disk of the complex plane

$$
D\left(\lambda_{k, i}^{\alpha, 0}, 2|a|\right)
$$

where conventionally $\lambda_{k, i}^{\alpha, 0}:=\lambda_{k, i}^{\alpha}$, the eigenvalues of our self-adjoint operator $\mathcal{A}_{\alpha}$.

Proof The solutions $\mu_{k, i}^{\alpha, a}$ of the transcendental equation $f(\mu)=0$ are the square roots of the eigenvalues of our operator. Thus we can set $\lambda_{k, i}^{\alpha, a}=\left(\mu_{k, i}^{\alpha, a}\right)^{2}$.

Moreover, for $k$ large enough, we have for $i=1,2$, that

$$
d\left(\lambda_{k, i}^{\alpha, 0}, \Lambda_{\alpha, 0} \backslash\left\{\lambda_{k, i}^{\alpha, 0}\right\}\right) \geq 4|a|
$$

so that, we have the resolvent estimate

$$
\left\|\left(\mathcal{A}_{\alpha}^{*}-\xi I\right)^{-1}\right\| \leq \frac{1}{2|a|}, \quad \forall \xi \in \partial D\left(\lambda_{k, i}^{\alpha, 0}, 2|a|\right),
$$

and thus

$$
\left\|\mathcal{M}_{a}^{*}\right\|\left\|\left(\mathcal{A}_{\alpha}^{*}-\xi I\right)^{-1}\right\| \leq \frac{1}{2}, \quad \forall \xi \in \partial D\left(\lambda_{k, i}^{\alpha, 0}, 2|a|\right) .
$$

From [31, IV-Theorems 3.16, 3.18, and V-§4.3], we know that the perturbed operator $\mathcal{A}_{\alpha, a}^{*}$ and the self-adjoint operator $\mathcal{A}_{\alpha}^{*}$ have the same number of eigenvalues in the disk $D\left(\lambda_{k, i}^{\alpha, 0}, 2|a|\right)$. Therefore, in this disk, $\mathcal{A}_{\alpha, a}^{*}$ has only one eigenvalue which is $\lambda_{k, i}^{\alpha, a}$. 
Conclusion on the structure of $\Lambda_{\alpha, a}$. Using the fact given by (4.8) and the Corollary 4.5 , we deduce that the spectrum of $\mathcal{A}_{\alpha, a}^{*}$ can be split into two disjoint parts

$$
\Lambda_{\alpha, a}=\Lambda_{\alpha, a}^{0} \cup \Lambda_{\alpha, a}^{\infty},
$$

where $\Lambda_{\alpha, a}^{0}$ is finite, with possibly some complex eigenvalues, and satisfy

$$
\Lambda_{\alpha, a}^{0} \subset \bigcup_{i=1,2} \bigcup_{0 \leq k<k_{\alpha, a}} D\left(\lambda_{k, i}^{\alpha, 0}, 2|a|\right)
$$

and $\Lambda_{\alpha, a}^{\infty} \subset(0,+\infty)$ and is defined by

$$
\Lambda_{\alpha, a}^{\infty}:=\left\{\lambda_{k, 1}^{\alpha, a}, k \geq k_{\alpha, a}\right\} \cup\left\{\lambda_{k, 2}^{\alpha, a}, k \geq k_{\alpha, a}\right\} .
$$

The situation is illustrated in Figure 4.1. This image has been obtained by numerically computing the spectrum of the discretized operator $\mathcal{A}_{\alpha, a}^{*}$ on a very refined mesh (see Section 5.2 for details about the discretization procedure).

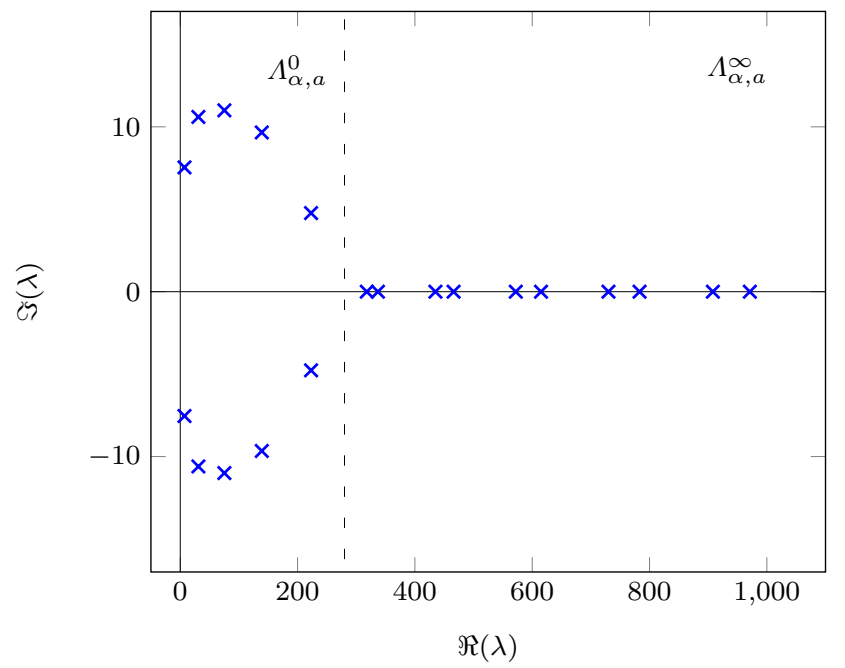

Fig. 4.1 A numerical description of a part of the spectrum: for $a=30, \alpha=0.1$

We can summarize the above analysis as follows.

Proposition 4.6 Let $a \in \mathbb{R}$ and $\alpha \geq 0$ be any two parameters.

- The spectrum of the operator $\mathcal{A}_{\alpha, a}^{*}$ is discrete, made only of simple eigenvalues, and has the structure given in (4.18).

- Moreover, the associated family of eigenfunctions $\left\{\Phi_{\lambda}\right\}_{\lambda \in \Lambda_{\alpha, a}}$ is complete in $E$ and $\mathcal{H}_{\alpha}$.

Note that we considered here the complex version of the spaces $E$ and $\mathcal{H}_{\alpha}$. Everything was proved above, except the completeness property of the eigenfunctions which comes as a consequence of a theorem of Keldysh, see for instance [38, Chapter 1 -Theorem 4.3], since the perturbation $\mathcal{M}_{a}^{*}$ is bounded. 
4.2 Observation estimates and bounds on norms of eigenfunctions

In this section, we analyze the size of the observation terms $\left|\mathcal{B}_{2}^{*} \Phi_{\lambda}\right|$ for $\lambda \in \Lambda_{\alpha, a}$ $\left(\mathcal{B}_{2}^{*}\right.$ is defined by $\left.(2.13 \mathrm{~b})\right)$. If those quantities do not vanish then the approximate controllability of the problem $(1.7)-(1.8 \mathrm{~b})$ will be guaranteed by means of Fattorini-Hautus test (see $[25,39]$ ). Moreover, suitable lower bound for those quantities combined with upper bounds of $\left\|\Phi_{\lambda}\right\|_{\mathcal{H}_{\alpha}}$ will let us build and estimate a null-control in $L^{2}(0, T)$ via moments technique.

\subsubsection{Approximate controllability}

We prove the following lemma (recall that we have assumed the diffusion coefficients $\gamma_{1}=\gamma_{2}=1$ ).

Lemma 4.7 There exists a non-empty set $\mathcal{R} \subset \mathbb{R}_{0}^{+} \times \mathbb{R}^{*}$ of null measure, such that we have the following properties:

1. If $(\alpha, a) \notin \mathcal{R}$, the problem $(1.7)-(1.8 \mathrm{~b})$ is approximately controllable at any time $T>0$ in $\mathcal{H}_{-\alpha}$.

2. On the other hand, if $(\alpha, a) \in \mathcal{R}$, there exists a subspace $\mathcal{Y}_{\alpha, a} \subset \mathcal{H}_{-\alpha}$ of codimension one, such that the problem (1.7)-(1.8b) is approximately controllable at any time $T>0$ if and only if the initial data belongs to $\mathcal{Y}_{\alpha, a}$.

The set $\mathcal{R}$ and the spaces $\mathcal{Y}_{\alpha, a}$ are defined by (4.29) and (4.31) respectively inside the proof of this lemma.

Proof (of Lemma 4.7)

We recall that the observation operator $\mathcal{B}_{2}^{*}$ is given in $(2.13 \mathrm{~b})$.

- In the simplest case when $a=0$, for any $\alpha \geq 0$, one can immediately see that the eigenfunctions in (4.5)-(4.6) satisfy

$$
\mathcal{B}_{2}^{*} \Phi_{\lambda_{k, 1}^{\alpha}}=\sqrt{\lambda_{k, 1}^{\alpha}} \neq 0, \quad \mathcal{B}_{2}^{*} \Phi_{\lambda_{k, 2}^{\alpha}}=\sqrt{\lambda_{k, 2}^{\alpha}}=(k+1) \pi \neq 0, \quad \forall k \geq 0
$$

- The case $\lambda=0$ can only happen if $a+3 \alpha+6=0$ (so that in particular $a<0$ ) and it follows from (4.9) that

$$
\mathcal{B}_{2}^{*} \Phi_{0}=1-\frac{a}{6}>0
$$

- Let us assume that $a \neq 0$ and $\lambda \neq 0$ be an eigenvalue of $\mathcal{A}_{\alpha, a}^{*}$. The associated eigenfunction $\Phi_{\lambda}$ is given in (4.13).

An immediate computation gives

$$
\mathcal{B}_{2}^{*} \Phi_{\lambda}=-\frac{a}{2 \mu}+\mu+\frac{a \cos \mu}{2 \sin \mu}
$$

From now on, we suppose that $\mathcal{B}_{2}^{*} \Phi_{\lambda}=0$. Since $\mu \neq 0$ and $\sin \mu \neq 0$, this is equivalent to the relation

$$
\left(2 \mu^{2}-a\right) \sin \mu+a \mu \cos \mu=0 .
$$


This equation has to be satisfied in addition to the transcendental equation (4.12). If we suppose that $\cos \mu=0$, then (4.12) and (4.23) show that this can occur if and only if we have

$$
a+2 \alpha=0, \text { and } \mu^{2}=-\alpha,
$$

this last equation not being compatible with the condition $\cos \mu=0$.

Therefore, we can assume that $\cos \mu \neq 0$. Multiplying (4.23) by $2 \cos \mu$ and using straightforward trigonometry we obtain that the two equations (4.12) and (4.23) can be equivalently written as follows

$$
\left(\begin{array}{cc}
\left(4 \mu^{2}-a\right) & 4 \alpha \mu \\
\left(2 \mu^{2}-a\right) & -2 a \mu
\end{array}\right)\left(\begin{array}{c}
\sin 2 \mu \\
\sin ^{2} \mu
\end{array}\right)=\left(\begin{array}{l}
-2 a \mu \\
-2 a \mu
\end{array}\right)
$$

Denote the coefficient matrix in the left hand side of (4.25) by $M_{\mu} \in M_{2 \times 2}(\mathbb{C})$ and we calculate the determinant:

$$
\operatorname{det} M_{\mu}=2 a \mu(a+2 \alpha)-8 \mu^{3}(a+\alpha) .
$$

- Let us prove that $\operatorname{det} M_{\mu} \neq 0$ if $\mu$ satisfies (4.25).

The claim is clear if $a+2 \alpha=0$ or $a+\alpha=0$, since $a$ and $\mu$ are non-zero.

From now on we assume $a+2 \alpha \neq 0$ and $a+\alpha \neq 0$. The determinant of $M_{\mu}$ cancels if and only if $\mu= \pm \sqrt{\frac{a(a+2 \alpha)}{4(a+\alpha)}} \in \mathbb{C}$ and, if that happens, the matrix becomes

$$
M_{\mu}=\left(\begin{array}{cc}
\frac{a \alpha}{a+\alpha} & \pm 2 \alpha \sqrt{\frac{a(a+2 \alpha)}{(a+\alpha)}} \\
-\frac{1}{2} \frac{a^{2}}{a+\alpha} & \mp a \sqrt{\frac{a(a+2 \alpha)}{(a+\alpha)}}
\end{array}\right),
$$

and a straightforward computation shows that

$$
\operatorname{Ker} M_{\mu}^{*}=\operatorname{Span}\left\{\left(\begin{array}{c}
a \\
2 \alpha
\end{array}\right)\right\} \text {. }
$$

We deduce from (4.25) that $\left(\begin{array}{l}1 \\ 1\end{array}\right)$ belongs to the range of $M_{\mu}$, which implies that it should be orthogonal to $\operatorname{Ker} M_{\mu}^{*}$, that is

$$
\left(\begin{array}{l}
1 \\
1
\end{array}\right) \perp\left(\begin{array}{c}
a \\
2 \alpha
\end{array}\right)
$$

This is a contradiction since $a+2 \alpha \neq 0$ and the claim is proved.

- Solving (4.25).

From the previous point, we know that $M_{\mu}$ is invertible, so that we can solve (4.25) to get

$$
\left\{\begin{array}{l}
\sin 2 \mu=\frac{2 a \mu(a+2 \alpha)}{a(a+2 \alpha)-4 \mu^{2}(a+\alpha)} \\
\sin ^{2} \mu=\frac{-2 a \mu^{2}}{a(a+2 \alpha)-4 \mu^{2}(a+\alpha)} .
\end{array}\right.
$$


Using the standard trigonometric relation $\sin ^{2} 2 \mu=4 \sin ^{2} \mu\left(1-\sin ^{2} \mu\right)$, we can deduce from (4.26) that

$$
4 \mu^{2}=a(a+2 \alpha+2) .
$$

Since the sign of $\mu$ is unimportant, we conclude that this situation can only occur for the particular value

$$
\mu=\mu_{\alpha, a}^{c}:=\frac{1}{2} \sqrt{a^{2}+2 a \alpha+2 a} .
$$

To summarize, we have finally obtained that if $\mathcal{B}_{2}^{*} \Phi_{\lambda}=0$, then we necessarily have

$$
\lambda=\lambda_{\alpha, a}^{c}:=\frac{a(a+2 \alpha+2)}{4} .
$$

This is a necessary condition and we still have to check whether or not this value of $\lambda$ (or $\mu$ ) does satisfy (4.26), that is to say if $\alpha$ and $a$ satisfy

$$
\begin{aligned}
\sin \left(\sqrt{a^{2}+2 a \alpha+2 a}\right) & =-\frac{(a+2 \alpha) \sqrt{a^{2}+2 a \alpha+2 a}}{\left(a^{2}+a+3 a \alpha+2 \alpha^{2}\right)}, \\
\sin ^{2}\left(\frac{\sqrt{a^{2}+2 a \alpha+2 a}}{2}\right) & =\frac{\left(a^{2}+2 a \alpha+2 a\right)}{2\left(a^{2}+a+3 a \alpha+2 \alpha^{2}\right)} .
\end{aligned}
$$

This leads us to introduce the critical set $\mathcal{R}$ as follows

$$
\mathcal{R}:=\left\{(\alpha, a) \in \mathbb{R}_{0}^{+} \times \mathbb{R}^{*}, \text { s.t. (4.28) holds }\right\}
$$

The set $\mathcal{R}$ is the set of solutions to the two equations (4.28). We recall that those two equations were obtained from (4.26) by eliminating the value of $\mu$ and therefore, are not independent one from the other. Thus, we observe that any solution (4.28b) necessarily satisfies

$$
\sin \left(\sqrt{a^{2}+2 a \alpha+2 a}\right)=\varepsilon \frac{(a+2 \alpha) \sqrt{a^{2}+2 a \alpha+2 a}}{\left(a^{2}+a+3 a \alpha+2 \alpha^{2}\right)},
$$

for $\varepsilon \in\{-1,1\}$. On any connected component of the set of solutions of (4.28b), we have either $\varepsilon=-1$ (in which case (4.28a) is satisfied) or $\varepsilon=1$ (in which case (4.28a) is not satisfied).

We have plotted in Figure 4.2 the solution curves of $(4.28 \mathrm{~b})$ in two colors: in blue the ones for which $\varepsilon=-1$ and in red the ones for which $\varepsilon=1$. The set $\mathcal{R}$ is thus the union of the blue curves. The blue dot corresponds to the particular pair $(\alpha, a)=(1,3.1931469)$ that is used in the numerical results of Section 5.3.

- To sum up the previous analysis, we have identified the set $\mathcal{R}$ of parameters $(\alpha, a)$ for which there exists a single critical eigenvalue $\lambda_{\alpha, a}^{c}$ given by (4.27) for which the associated eigenfunction is not observable, that is $\mathcal{B}_{2}^{*} \Phi_{\lambda_{\alpha, a}^{c}}=0$.

We can now find out the approximate controllability properties of our problem.

1 . For any given pair $(\alpha, a) \notin \mathcal{R}$ all the eigenfunctions of $\mathcal{A}_{\alpha, a}^{*}$ are observable, and henceforth, the Fattorini-Hautus criterion is satisfied (see [25, 39]) which implies the approximate controllability of the system in the space $\mathcal{H}_{-\alpha}$. 


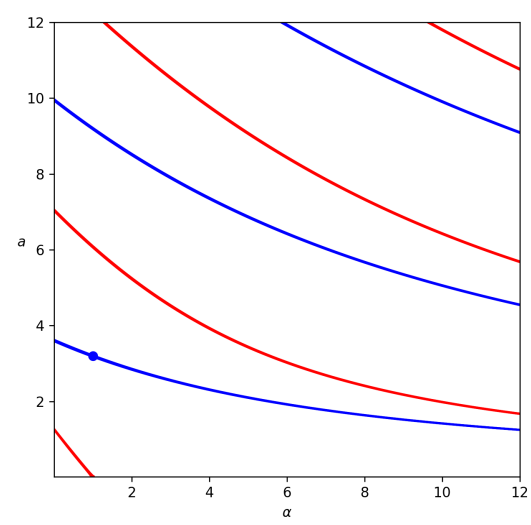

Fig. 4.2 In blue: the set $\mathcal{R}$ of critical pairs $(\alpha, a)$. In red: The solutions to (4.28b) that are not solution of (4.28a).

2. If a given pair $(\alpha, a)$ belongs to $\mathcal{R}$, then the system (1.7)-(1.8b) cannot be approximately controllable in the full space $\mathcal{H}_{-\alpha}$, since for the particular eigenvalue given in $(4.27)$, we have $\mathcal{B}_{2}^{*} \Phi_{\lambda_{\alpha, a}^{c}}=0$; thus the Fattorini-Hautus criterion fails.

However, it is not difficult to observe that if the initial data belongs to the smaller space defined by

$$
\mathcal{Y}_{\alpha, a}:=\left\{y_{0} \in \mathcal{H}_{-\alpha} \mid\left\langle y_{0}, \Phi_{\lambda_{\alpha, a}^{c}}\right\rangle_{\mathcal{H}_{-\alpha}, \mathcal{H}_{\alpha}}=0\right\}
$$

then the approximate controllability of the system holds true.

\subsubsection{Estimates on the eigenfunctions}

We will gather here the estimates we need on the eigenfunctions, namely a bound from below for the observation terms $\mathcal{B}_{2}^{*} \Phi_{\lambda}$ and a bound from above for the norms $\left\|\Phi_{\lambda}\right\|_{\mathcal{H}_{\alpha}}$.

Lemma 4.8 Let $a \in \mathbb{R}$ and $\alpha \geq 0$ be given. Then, there exists some $C_{\alpha, a}$ such that we have

$$
\left\|\Phi_{\lambda}\right\|_{\mathcal{H}_{\alpha}} \leq C_{\alpha, a}(1+\sqrt{|\lambda|}), \quad \forall \lambda \in \Lambda_{\alpha, a},
$$

and moreover, the observation terms enjoy the following estimate

1. When $(\alpha, a) \notin \mathcal{R}$, we have

$$
\left|\mathcal{B}_{2}^{*} \Phi_{\lambda}\right| \geq \frac{1}{C_{\alpha, a}}(1+\sqrt{|\lambda|}), \quad \forall \lambda \in \Lambda_{\alpha, a}
$$

2. On the other hand, for any pair $(\alpha, a) \in \mathcal{R}$, we have the same estimate (4.32) for all $\lambda \in \Lambda_{\alpha, a} \backslash\left\{\lambda_{\alpha, a}^{c}\right\}$, where $\lambda_{\alpha, a}^{c}$ is given in (4.27). 
Proof We first observe that, thanks to the structure of the spectrum of our operator given in (4.18), it is enough to establish the required estimates for $\lambda \in \Lambda_{\alpha, a}^{\infty}$, in which case we can take advantage of the explicit asymptotic behavior of the eigenvalues that we have established above. Moreover we only treat here the case $a \neq 0$ since the case $a=0$ can be treated easily in the very same way by using formulas (4.5)-(4.6) instead of (4.13).

- For the case $\lambda=0$, (which is possible only when $a+3 \alpha+6=0$ ) it is easy to see from (4.9) that there exists some $C_{\alpha, a}>0$ such that

$$
\left\|\Phi_{0}\right\|_{\mathcal{H}_{\alpha}} \leq C_{\alpha, a}
$$

- Next, we suppose $\lambda \neq 0$ and observe that

$$
\begin{aligned}
\left\|\Phi_{\lambda}\right\|_{\mathcal{H}_{\alpha}}^{2} & =\left(\mathcal{A}_{\alpha, 0} \Phi_{\lambda}, \Phi_{\lambda}\right)_{E} \\
& =\left(\mathcal{A}_{\alpha, a}^{*} \Phi_{\lambda}, \Phi_{\lambda}\right)_{E}-\left(\mathcal{M}_{a}^{*} \Phi_{\lambda}, \Phi_{\lambda}\right)_{E} \\
& =\lambda\left\|\Phi_{\lambda}\right\|_{E}^{2}-\left(\mathcal{M}_{a}^{*} \Phi_{\lambda}, \Phi_{\lambda}\right)_{E} \\
& \leq(|a|+|\lambda|)\left\|\Phi_{\lambda}\right\|_{E}^{2} .
\end{aligned}
$$

Therefore, we are reduced to find a uniform estimate of the norm in $E$ of $\Phi_{\lambda}$. Using the explicit expression (4.13) of the eigenfunction $\Phi_{\lambda}$ we get that

$$
\left\|\Phi_{\lambda}\right\|_{E} \leq C_{a}\left(1+\frac{1}{|\mu \sin \mu|}\right)
$$

and so we finally simply need to show that

$$
\sup _{k \geq k_{\alpha, a}}\left\|\Phi_{\lambda_{k, 1}^{\alpha, a}}\right\|_{E}+\sup _{k}\left\|\Phi_{\lambda_{k, 2}^{\alpha, a}}\right\|_{E}<+\infty .
$$

- Concerning the first family of eigenvalues, by the asymptotics (4.14), we have that $\left|\sin \left(\mu_{k, 1}^{\alpha, a}\right)\right|$ is close to 1 for $k$ large enough. Therefore it is clear that $\left\|\Phi_{\lambda_{k, 1}^{\alpha, a}}\right\|_{E}$ is a bounded quantity when $k$ goes to infinity.

- For the second family of eigenvalues, using (4.15) we see that $\sin \left(\mu_{k, 2}^{\alpha, a}\right)$ is now close to 0 for $k$ large. However, the precise asymptotics shows that the product

$$
\left|\mu_{k, 2}^{\alpha, a} \sin \left(\mu_{k, 2}^{\alpha, a}\right)\right|
$$

is close to $|a| / 4$ for $k$ large, and thus $\left\|\Phi_{\lambda_{k, 2}^{\alpha, a}}\right\|_{E}$ is also bounded.

- Concerning the observation terms, we start from (4.22) and separate again the study for the two families of eigenvalues.

- By the same argument as before, we see that $\left|\sin \left(\mu_{k, 1}^{\alpha, a}\right)\right|$ is close to 1 and $\left|\cos \left(\mu_{k, 1}^{\alpha, a}\right)\right|$ is close to 0 , so that we get

$$
\mathcal{B}_{2}^{*} \Phi_{\lambda_{k, 1}^{\alpha, a}+\infty}^{\sim} \mu_{k, 1}^{\alpha, a}=\sqrt{\lambda_{k, 1}^{\alpha, a}}
$$

- Concerning the second family of eigenvalues, we need to carefully study the last term in (4.22), that gives

$$
\frac{a \cos \left(\mu_{k, 2}^{\alpha, a}\right)}{2 \sin \left(\mu_{k, 2}^{\alpha, a}\right)} \underset{+\infty}{\sim} \frac{a(-1)^{k+1}}{2(-1)^{k+1}\left(\frac{-a}{4 k \pi}\right)}=-2 k \pi \underset{+\infty}{\sim}-2 \mu_{k, 2}^{\alpha, a} .
$$


Coming back to (4.22), we conclude that

$$
\mathcal{B}_{2}^{*} \Phi_{\lambda_{k, 2}^{\alpha, a}+\infty}^{\sim}-\mu_{k, 2}^{\alpha, a}=-\sqrt{\lambda_{k, 2}^{\alpha, a}}
$$

This, along with Lemma 4.7 gives the required results in points 1 and 2 of our Lemma.

\subsection{Null-controllability}

We now focus on obtaining a null-control for the system (1.7)-(1.8b). We recall again that the diffusion coefficients are $\gamma_{1}=\gamma_{2}=1$.

\subsubsection{The moments problem}

The set of eigenfunctions $\left\{\Phi_{\lambda}\right\}_{\lambda \in \Lambda_{\alpha, a}}$ of $\mathcal{A}_{\alpha, a}^{*}$ is a complete family in $\mathcal{H}_{\alpha}$ on account of Proposition 4.6, so it is enough to check the controllability equation (2.12) for $\Phi_{\lambda}$ for each $\lambda \in \Lambda_{\alpha, a}$. This indeed tells us that, for any $y_{0} \in \mathcal{H}_{-\alpha}$, the input $v \in L^{2}(0, T ; \mathbb{C})$ is a null-control for $(1.7)-(1.8 \mathrm{~b})$ if and only if we have

$$
-\overline{e^{-\lambda T}}\left\langle y_{0}, \Phi_{\lambda}\right\rangle_{\mathcal{H}_{-\alpha}, \mathcal{H}_{\alpha}}=\overline{\mathcal{B}_{2}^{*} \Phi_{\lambda}} \int_{0}^{T} v(t) \overline{e^{-\lambda(T-t)}} d t, \quad \forall \lambda \in \Lambda_{\alpha, a} .
$$

where we used the fact that $e^{-t \mathcal{A}_{\alpha, a}^{*}} \Phi_{\lambda}=e^{-t \lambda} \Phi_{\lambda}$, for any $\lambda \in \Lambda_{\alpha, a}$.

The above set of equations are the moments problem in our case, that we need to solve.

\subsubsection{Existence of bi-orthogonal family}

From the set of moments problem, we shall find a control $v$ built upon a suitable biorthogonal family to the time-dependent exponential functions. In this context, it is worth mentioning [8, Theorem 1.5] where the authors proved the existence of biorthogonal families to $\left\{t^{j} e^{-\sigma_{k} t}\right\}_{k \geq 0,0 \leq j \leq \eta}(\eta \in \mathbb{N})$ for a complex sequence $\left\{\sigma_{k}\right\}_{k \geq 0}$ with non-decreasing modulus. This proof is based on a proper gap condition of $\left|\sigma_{k}-\sigma_{n}\right|$ for all $k \neq n$ and some property of the counting function associated with $\left\{\sigma_{k}\right\}_{k \geq 0}$. In fact, concerning this hypothesis on the counting function, a slightly modified version has been introduced in [2, Remark 4.3] and below, we write a simpler version of [8, Theorem 1.5] combined with [2, Remark 4.3] which will be used in our case.

Theorem 4.9 Let $\left\{\sigma_{k}\right\}_{k \geq 0} \subset \mathbb{C}$ be a sequence of complex numbers fulfilling the following assumptions:

1. $\sigma_{k} \neq \sigma_{n}$ for all $k, n \in \mathbb{N} \cup\{0\}$, with $k \neq n$;

2. $\Re\left(\sigma_{k}\right)>0$, for all $k \geq 0$ and there exists some constant $c>0$, independent on $k \geq 0$, such that

$$
\left|\Im\left(\sigma_{k}\right)\right| \leq c \sqrt{\Re\left(\sigma_{k}\right)}, \quad \forall k \geq 0 ;
$$

3. $\left\{\sigma_{k}\right\}_{k \geq 0}$ is non-decreasing in modulus, that is

$$
\left|\sigma_{k+1}\right| \geq\left|\sigma_{k}\right|, \quad \forall k \geq 0
$$


4. $\left\{\sigma_{k}\right\}_{k \geq 0}$ satisfies the following gap condition: there exists some $\rho>0$ and $p \in \mathbb{N}$ such that

$$
\left\{\begin{array}{l}
\left|\sigma_{k}-\sigma_{n}\right| \geq \rho\left|k^{2}-n^{2}\right|, \quad \forall k, n:|k-n| \geq p \\
\inf _{k \neq n:|k-n|<p}\left|\sigma_{k}-\sigma_{n}\right|>0
\end{array}\right.
$$

5. for some $\theta>0$ and $c_{\min }, c_{\max }>0$, the counting function $\mathcal{N}:(0,+\infty) \rightarrow(0,+\infty)$ corresponding to the set $\left\{\sigma_{k}\right\}_{k \geq 0}$,

$$
\mathcal{N}(r)=\#\left\{k:\left|\sigma_{k}\right| \leq r\right\}, \quad \forall r>0,
$$

satisfies the following inequality

$$
-\theta+c_{\min } \sqrt{r} \leq \mathcal{N}(r) \leq \theta+c_{\max } \sqrt{r}, \quad \forall r>0 ;
$$

Then, there exists $T_{0}>0$ such that, for $0<T \leq T_{0}$, we can find a family of complex valued functions $\left\{q_{k}\right\}_{k \geq 0} \subset L^{2}(0, T)$, bi-orthogonal to $\left\{e^{-\sigma_{k}(T-t)}\right\}_{k \geq 0}$, that is,

$$
\int_{0}^{T} q_{k}(t) \overline{e^{-\sigma_{l}(T-t)}} d t=\delta_{k, l} \quad \forall k, l \geq 0,
$$

with in addition,

$$
\left\|q_{k}\right\|_{L^{2}(0, T)} \leq C e^{C \sqrt{\Re\left(\sigma_{k}\right)}+\frac{C}{T}}, \text { for any } k \geq 0,
$$

with a constant $C:=C\left(c, \rho, \theta, c_{\min }, c_{\max }\right)>0$ which does not depend on the choice of sequence $\left\{\sigma_{k}\right\}_{k \geq 0}$.

We will show that the set of eigenvalues $\Lambda_{\alpha, a}$ satisfies all the assumptions of Theorem 4.9 to find the bi-orthogonal family for $\left\{e^{-\lambda(T-t)}\right\}_{\lambda \in \Lambda_{\alpha, a}}$.

Remark 4.10 According to Theorem 4.9, all the elements of $\Lambda_{\alpha, a}$ should have positive real part. So, if needed, one could choose some $m_{\alpha, a}>0$, such that $\left(\lambda+m_{\alpha, a}\right)$ has positive real part for all $\lambda \in \Lambda_{\alpha, a}$, and we shall then focus on finding the bi-orthogonal family for $\left\{e^{-\left(\lambda+m_{\alpha, a}\right)(T-t)}\right\}_{\lambda \in \Lambda_{\alpha, a}}$.

Thus, without loss of generality, we assume that the eigenvalues $\lambda \in \Lambda_{\alpha, a}$ have positive real parts.

1. We know that the set of eigenvalues $\Lambda_{\alpha, a}$ is discrete, and that only a finite number of them are possibly non-real, see (4.18). Therefore, we clearly have the estimate

$$
|\Im(\lambda)| \leq C_{\alpha, a} \sqrt{\Re(\lambda)}, \quad \forall \lambda \in \Lambda_{\alpha, a} .
$$

2. The gap condition

Since the spectrum of $\mathcal{A}_{\alpha, a}^{*}$ is discrete, one has $|\lambda-\widetilde{\lambda}| \neq 0$ for any two eigenvalues $\lambda \neq \tilde{\lambda}$ in $\Lambda_{\alpha, a}$. So, it is enough to obtain a proper gap condition for large eigenvalues where we take the advantage of having only real eigenvalues.

In this context, it is important to recall the set of all real eigenvalues defined in (4.20) and for simplicity one may re-denote the sequence $\left\{\lambda_{k, i}^{\alpha, a}\right\}_{k \geq k_{\alpha, a}, i=1,2}$ by $\left\{\lambda_{2 k+i}\right\}_{k \geq k_{\alpha, a}, i=1,2}\left(k_{\alpha, a}\right.$ has been introduced in Corollary 4.5), in increasing order as follows

$$
\lambda_{2 k_{\alpha, a}+1}<\lambda_{2 k_{\alpha, a}+2}<\lambda_{2\left(k_{\alpha, a}+1\right)+1}<\cdots,
$$


with

$$
\lambda_{2 k+1}:=\lambda_{k, 1}^{\alpha, a}, \quad \lambda_{2 k+2}:=\lambda_{k, 2}^{\alpha, a}, \quad \forall k \geq k_{\alpha, a} .
$$

Note that for the re-defined sequence above, we start with the index $2 k_{\alpha, a}+1$, the reason is that the set $\Lambda_{\alpha, a}^{0} \subset \Lambda_{\alpha, a}$ (see (4.19)) contains exactly $2 k_{\alpha, a}$ eigenvalues.

Let us take into account the asymptotic formulas given by Corollary 4.5 and compute the following,

$$
\begin{aligned}
\lambda_{2 k+2}-\lambda_{2 k+1}=\lambda_{k, 2}^{\alpha, a}-\lambda_{k, 1}^{\alpha, a} & =(k+1)^{2} \pi^{2}-\left(k+\frac{1}{2}\right)^{2} \pi^{2}+O_{\alpha, a}(1) \\
& \geq \widetilde{c}_{\alpha, a} k \pi^{2}, \quad(\text { for large } k) \\
& \geq \widetilde{c}_{\alpha, a} \frac{\pi^{2}}{7}\left[(2 k+2)^{2}-(2 k+1)^{2}\right]
\end{aligned}
$$

as also,

$$
\begin{aligned}
\lambda_{2(k+1)+1}-\lambda_{2 k+2}=\lambda_{k+1,1}^{\alpha, a}-\lambda_{k, 2}^{\alpha, a} & =\left(k+1+\frac{1}{2}\right)^{2} \pi^{2}-(k+1)^{2} \pi^{2}+O_{\alpha, a}(1) \\
& \geq \widetilde{c}_{\alpha, a}(k+1) \pi^{2}, \quad(\text { for large } k) \\
& \geq \widetilde{c}_{\alpha, a} \frac{\pi^{2}}{5}\left[(2(k+1)+1)^{2}-(2 k+2)^{2}\right]
\end{aligned}
$$

for some constant $\widetilde{c}_{\alpha, a}>0$. Now, from the above two inequalities, it is not difficult to obtain

$$
\lambda_{2 k+i}-\lambda_{2 n+j} \geq \rho_{\alpha, a}\left[(2 k+i)^{2}-(2 n+j)^{2}\right], \forall\left\{\begin{array}{l}
k>n \geq \tilde{k}_{\alpha, a}, i, j \in\{1,2\} \\
k=n \geq \tilde{k}_{\alpha, a}, i>j,
\end{array}\right.
$$

for some $\tilde{k}_{\alpha, a} \geq k_{\alpha, a}$ and with $\rho_{\alpha, a}=\widetilde{c}_{\alpha, a} \pi^{2} / 7$, independent on the choices of the eigenvalues. Hence, the requirement of gap condition satisfies.

3. The counting function

Let $\mathcal{N}$ be the counting function associated with the set of eigenvalues $\Lambda_{\alpha, a}$, defined by

$$
\mathcal{N}(r)=\#\left\{\lambda \in \Lambda_{\alpha, a}:|\lambda| \leq r\right\}, \quad \forall r>0 .
$$

We have that, the function $\mathcal{N}$ is piecewise constant and non-decreasing in the interval $[0,+\infty)$. Also for every $r \in(0,+\infty)$ we have $\mathcal{N}(r)<+\infty$ and $\lim _{r \rightarrow+\infty} \mathcal{N}(r)=+\infty$.

Without loss of generality, one can start with some sufficiently large number $\bar{r}>0$, such that $\forall r \geq \bar{r}$, the eigenvalue $\lambda_{\mathcal{N}(r)}$ is real. Assuming this $\mathcal{N}(r)$ to be an odd number, we have, from the definition of $\mathcal{N}$, that

$$
\begin{gathered}
\mathcal{N}(r)=2 k+1 \Longleftrightarrow \lambda_{2 k+1} \leq r \text { and } \lambda_{2 k+2}>r, \text { for } k \geq k_{\alpha, a}, \\
\text { i.e., } \sqrt{\lambda_{2 k+1}} \leq \sqrt{r}<\sqrt{\lambda_{2 k+2}}, \text { for } k \geq k_{\alpha, a},
\end{gathered}
$$

which yields, by Lemma 4.4,

$$
\left(k+\frac{1}{4}\right) \pi \leq \sqrt{r}<\left(k+\frac{5}{4}\right) \pi, \text { for } k \geq k_{\alpha, a} \text { and } \forall r \geq \bar{r}>0 .
$$


Replacing $k$ by $\frac{(\mathcal{N}(r)-1)}{2}$, we determine that

$$
\frac{2}{\pi} \sqrt{r}-\frac{3}{2}<\mathcal{N}(r) \leq \frac{2}{\pi} \sqrt{r}+\frac{1}{2}, \quad \forall r \geq \bar{r}>0 .
$$

Similarly, for even $\mathcal{N}(r)$, we shall have similar estimate for $\mathcal{N}(r)$.

Now, for smaller $0<r<\bar{r}$, it is obvious that there always exists some constant $\widehat{c}_{\alpha, a}>0$, sufficiently large and independent of $0<r<\bar{r}$ such that

$$
\mathcal{N}(r) \leq \widehat{c}_{\alpha, a}(\sqrt{r}+1)
$$

since $\mathcal{N}$ is a bounded function in $(0, \bar{r})$.

The above inequalities (4.35) and (4.36) are the required conditions for counting functions.

So, by virtue of [8, Theorem 1.5], we can ensure the existence of a family $\left\{q_{\lambda}\right\}_{\lambda \in \Lambda_{\alpha, a}} \subset L^{2}(0, T ; \mathbb{C})$, bi-orthogonal to $\left\{e^{-\lambda(T-t)}\right\}_{\lambda \in \Lambda_{\alpha, a}}, t \in(0, T)$, that is to say

$$
\int_{0}^{T} q_{\lambda}(t) \overline{e^{-\widetilde{\lambda}(T-t)}} d t=\delta_{\lambda, \tilde{\lambda}}, \quad \forall \lambda, \widetilde{\lambda} \in \Lambda_{\alpha, a}
$$

In addition, this family satisfies the following estimate

$$
\left\|q_{\lambda}\right\|_{L^{2}(0, T)} \leq C_{\alpha, a} e^{C_{\alpha, a}\left(\sqrt{\Re(\lambda)}+\frac{1}{T}\right)}, \quad \forall \lambda \in \Lambda_{\alpha, a}
$$

for some $C_{\alpha, a}>0$ which only does depend the constants obtained in the points 1 , 2,3 in the above discussions but definitely not on the eigenvalues $\lambda \in \Lambda_{\alpha, a}$.

\subsubsection{Existence of a control}

Now we are in the situation to prove the null-controllability result of (1.7)-(1.8b), typically the following proof.

\section{Proof (of Theorem 1.3)}

Without loss of generality, we prove the theorem for given time $0<T \leq 1$. Since for any time $\widetilde{T}>1$, we know that a continuation by 0 of a control in $(0,1)$ will still be a control in $(0, \widetilde{T})$.

- We first suppose $(\alpha, a) \notin \mathcal{R}$ and consider

$$
\begin{aligned}
& v(t)=\sum_{\lambda \in \Lambda_{\alpha, a}} v_{\lambda}(t), \quad \forall t \in(0, T), \quad \text { with } \\
& v_{\lambda}(t)=-\frac{\overline{e^{-\lambda T}}}{\overline{\mathcal{B}_{2}^{*} \Phi_{\lambda}}}\left\langle y_{0}, \Phi_{\lambda}\right\rangle_{\mathcal{H}_{-\alpha}, \mathcal{H}_{\alpha}} q_{\lambda}(t), \quad \forall t \in(0, T),
\end{aligned}
$$

for $\lambda \in \Lambda_{\alpha, a}$, any given $y_{0} \in \mathcal{H}_{-\alpha}$ and any $0<T \leq 1$. The above construction of $v_{\lambda}$ is well-defined since we have, by Lemma 4.7 , that $\mathcal{B}_{2}^{*} \Phi_{\lambda} \neq 0, \forall \lambda \in \Lambda_{\alpha, a}$. 
With this choice of $v$, we can observe that the set of moments problem (4.33) is formally satisfied. It remains to show the convergence of the series, and here we need to find the $L^{2}(0, T)$ norm of $v_{\lambda}$ for each $\lambda \in \Lambda_{\alpha, a}$. We see that

$$
\begin{aligned}
\left\|v_{\lambda}\right\|_{L^{2}(0, T)} & \leq \frac{\left|e^{-\lambda T}\right|}{\left|\mathcal{B}_{2}^{*} \Phi_{\lambda}\right|}\left\|y_{0}\right\|_{\mathcal{H}_{-\alpha}}\left\|\Phi_{\lambda}\right\|_{\mathcal{H}_{\alpha}}\left\|q_{\lambda}\right\|_{L^{2}(0, T)} \\
& \leq C_{\alpha, a} e^{\frac{C_{\alpha, a}}{T}} e^{-T \Re(\lambda)} e^{C_{\alpha, a} \sqrt{\Re(\lambda)+m_{\alpha, a}}} \times\left\|y_{0}\right\|_{\mathcal{H}_{-\alpha}} \frac{\left\|\Phi_{\lambda}\right\|_{\mathcal{H}_{\alpha}}}{\left|\mathcal{B}_{2}^{*} \Phi_{\lambda}\right|}
\end{aligned}
$$

thanks to the estimate of bi-orthogonal family in (4.37).

Thereafter, an application of Cauchy-Schwarz inequality gives

$$
C_{\alpha, a} \sqrt{\Re(\lambda)} \leq \frac{T}{2} \Re(\lambda)+\frac{C_{\alpha, a}^{2}}{2 T},
$$

so that one has

$$
e^{-T \Re(\lambda)} e^{C_{\alpha, a} \sqrt{\Re(\lambda)}} \leq e^{-\frac{T}{2} \Re(\lambda)+\frac{C_{\alpha, a}^{2}}{2 T}} \quad \forall \lambda \in \Lambda_{\alpha, a},
$$

Next, we use the estimates of the eigenfunctions from Lemma 4.8 to deduce

$$
\frac{\left\|\Phi_{\lambda}\right\|_{\mathcal{H}_{\alpha}}}{\left|\mathcal{B}_{2}^{*} \Phi_{\lambda}\right|} \leq C_{\alpha, a}, \quad \forall \lambda \in \Lambda_{\alpha, a}
$$

Now, take the sum over $\lambda \in \Lambda_{\alpha, a}$ in (4.39), then using the above bounds and applying (4.40), we obtain

$$
\sum_{\lambda \in \Lambda_{\alpha, a}}\left\|v_{\lambda}\right\|_{L^{2}(0, T)} \leq C_{\alpha, a} e^{\frac{C_{\alpha, a}}{T}}\left\|y_{0}\right\|_{\mathcal{H}_{-\alpha}} \sum_{\lambda \in \Lambda_{\alpha, a}} e^{-\frac{T}{2} \Re(\lambda)}
$$

We finally get that

$$
\|v\|_{L^{2}(0, T)} \leq C_{\alpha, a} e^{\frac{C_{\alpha, a}}{T}}\left\|y_{0}\right\|_{\mathcal{H}_{-\alpha}}
$$

with a constant $C_{\alpha, a}>0$ does not depend on $T$.

- On the other hand, when $(\alpha, a) \in \mathcal{R}$, we consider our control as

$$
v(t)=\sum_{\lambda \in \Lambda_{\alpha, a} \backslash\left\{\lambda_{\alpha, a}^{c}\right\}} v_{\lambda}(t), \quad \forall t \in(0, T),
$$

with the same formulation of $v_{\lambda}$ as prescribed in (4.38b).

Since we have assumed that $y_{0} \in \mathcal{Y}_{\alpha, a}$ (the space $\mathcal{Y}_{\alpha, a}$ has been defined in (4.31)), we see that the moments problem (4.33) is actually satisfied for any eigenvalue (in the case $\lambda=\lambda_{\alpha, a}^{c}$, both sides of the equality are zero).

The $L^{2}$-bound of this control alike (4.41) can be then obtained by a similar approach as previous. 


\section{Some numerical studies}

We devote this section to illustrate experimentally the controllability results shown in the previous sections and make experiments for some other control systems. We begin by presenting some facts about the classical penalized Hilbert Uniqueness Method (see e.g., [30,14]). Precisely, we shall accommodate [14, Proposition 1.5 and Theorem 1.7] in our functional setting which is not exactly the same as of the above references, as we recall that the weak solution $y$ to (1.7) with (1.8a), or $(1.8 \mathrm{~b})$, belongs to the space $\mathcal{C}^{0}\left([0, T] ; \mathcal{H}_{-\alpha}\right)$, where the abstract space $\mathcal{H}_{-\alpha}$ is larger than $E:=\left(L^{2}(0,1)\right)^{2}$. Next to this, we will introduce a general methodology to incorporate the effects of the boundary coupling(s) into the discretization of the system (1.1) (under the Assumption 1). We conclude this section by presenting several experiments.

\subsection{Adapting the penalized HUM approach}

Following the well-known penalized HUM approach, we shall look for the control $v$ minimizing the primal functional given by

$$
F_{\epsilon}(v):=\frac{1}{2} \int_{0}^{T}|v(t)|^{2} \mathrm{~d} t+\frac{1}{2 \epsilon}\|y(T)\|_{\mathcal{H}_{-\alpha}}^{2},
$$

where we used the same notation $y=\left(y_{1}, y_{2}\right)$ to denote the unique weak solution to the system (1.7) either with the boundary conditions (1.8a) or (1.8b) and for given initial data $y_{0} \in E$. For the sake of exposition, we deal with the system $(1.7)-(1.8 \mathrm{~b})$.

Observe that, for any $\epsilon>0$, the functional (5.1) has a unique minimizer in $L^{2}(0, T ; \mathbb{R})$ since $F_{\epsilon}$ is continuous, strictly convex and coercive. Hereafter, we denote this minimizer by $v_{\epsilon}$.

Using Fenchel-Rockafellar theory (see for instance [23]), we can identify an associated dual functional: for any $\epsilon>0$, we consider

$$
J_{\epsilon}(\zeta):=\frac{1}{2} \int_{0}^{T}\left|\mathcal{B}_{2}^{*} e^{-(T-t) \mathcal{A}_{\alpha, a}^{*} \zeta}\right|^{2} \mathrm{~d} t+\frac{\epsilon}{2}\|\zeta\|_{\mathcal{H}_{\alpha}}^{2}+\left(y_{0}, e^{-T \mathcal{A}_{\alpha, a}^{*} \zeta}\right)_{E},
$$

for all $\zeta:=\left(\zeta_{1}, \zeta_{2}\right) \in \mathcal{H}_{\alpha}$. We choose the initial data $y_{0} \in E$ and also recall the observation operator $\mathcal{B}_{2}^{*}$ defined by $(2.13 \mathrm{~b})$ associated with the control problem (1.7)-(1.8b).

For any $\epsilon>0$, the dual functional (5.2) also has a unique minimizer, that we denote by $\zeta^{\epsilon}$. Note that, in this case the coercivity comes from the term $\frac{\epsilon}{2}\|\zeta\|_{\mathcal{H}_{\alpha}}^{2}$ which corresponds, by duality, to the penalty term introduced in $F_{\epsilon}$.

Now, we characterize the minimizers $v_{\epsilon}$ and $\zeta^{\epsilon}$ in the following way.

Proposition 5.1 For any $\epsilon>0$, the minimizers $v_{\epsilon}$ and $\zeta^{\epsilon}$ of the functionals $F_{\epsilon}$ and $J_{\epsilon}$ respectively, are related through the formula

$$
v_{\epsilon}(t)=\mathcal{B}_{2}^{*} e^{-(T-t) \mathcal{A}_{\alpha, a}^{*} \zeta^{\epsilon}} \quad \text { for a.e. } t \in(0, T),
$$

and moreover, we have

$$
y^{\epsilon}(T, \cdot)=-\epsilon \mathcal{A}_{\alpha} \zeta^{\epsilon},
$$


where $y^{\epsilon}$ is the associated weak solution to $(1.7)-(1.8 \mathrm{~b})$ with control $v_{\epsilon}$ and initial data $y_{0} \in E$. As a consequence, we have

$$
\inf _{L^{2}(0, T ; \mathbb{R})} F_{\epsilon}=F_{\epsilon}\left(v_{\epsilon}\right)=-J_{\epsilon}\left(\zeta^{\epsilon}\right)=-\inf _{\mathcal{H}_{\alpha}} J_{\epsilon}
$$

We can prove this proposition by following a similar approach as [14, Proposition 1.5]; for a rigorous proof we refer to [11, Proposition IV.5.1].

The following result allows us to relate the controllability properties of system (1.7)-(1.8b) with the behavior of the minimizers shown above. More precisely, we write the following theorem.

Theorem 5.2 Let $v_{\epsilon}$ and $y^{\epsilon}$ be as in Proposition 5.1. Then we have the following.

- System (1.7)-(1.8b) is approximately controllable at time $T$ if and only if

$$
y^{\epsilon}(T) \rightarrow 0, \quad \text { as } \epsilon \rightarrow 0 .
$$

- System (1.7)-(1.8b) is null-controllable at time $T$ if and only if

$$
\mathcal{M}^{2}:=2 \sup _{\epsilon>0}\left(\inf _{L^{2}(0, T ; \mathbb{R})} F_{\epsilon}\right)<+\infty .
$$

In this case, we have

$$
\left\|v_{\epsilon}\right\|_{L^{2}(0, T ; \mathbb{R})} \leq \mathcal{M}, \quad\left\|y^{\epsilon}(T)\right\|_{\mathcal{H}_{-\alpha}} \leq \mathcal{M} \sqrt{\epsilon} .
$$

Moreover, the HUM control satisfies $\left\|v_{0}\right\|_{L^{2}(0, T ; \mathbb{R})}=\mathcal{M}$, and

$$
v_{\epsilon} \stackrel{\epsilon \rightarrow 0}{\longrightarrow} v_{0} \quad \text { strongly in } L^{2}(0, T ; \mathbb{R}) .
$$

The proof of such result follows from an adaptation of [14, Theorem 1.7], also we refer to [11, Theorem IV.5.2]. Let us remark that the supremum in (5.4) corresponds actually to the limit as $\epsilon \rightarrow 0$ of $\inf _{L^{2}(0, T ; \mathbb{R})} F_{\epsilon}$.

The main relevance is that the above theorem allows us to recapture the controllability results presented in the previous sections, using the constructive approach of the penalized HUM instead of other more involved arguments. At the numerical level this will be important since we expect that upon discretization the corresponding system maintains its controllability properties and Theorem 5.2 will help to conclude and illustrate this fact.

Now, we discuss some details about the implementation we follow to obtain the controls for the system (1.7)-(1.8b). We first define a bounded, non-negative, symmetric (w.r.t the duality product between $\mathcal{H}_{\alpha}$ and $\mathcal{H}_{-\alpha}$ ) operator $\Lambda: \mathcal{H}_{\alpha} \rightarrow$ $\mathcal{H}_{-\alpha}$ (usually referred to as the Gramian operator) as follows

$$
\Lambda \zeta=w^{\zeta}(T), \quad \forall \zeta \in \mathcal{H}_{\alpha},
$$

where the function $w^{\zeta}=\left(w_{1}^{\zeta}, w_{2}^{\zeta}\right) \in \mathcal{C}^{0}\left([0, T] ; \mathcal{H}_{-\alpha}\right)$ is obtained from $\zeta \in \mathcal{H}_{\alpha}$ as follows: we solve first the adjoint system (3.1) (with its solution $q(t, x)=$ $e^{\left.-(T-t) \mathcal{A}_{\alpha, a}^{*} \zeta(x)\right)}$, and then solve

$$
\begin{cases}\partial_{t} w_{1}-\partial_{x}\left(\gamma_{1} \partial_{x} w_{1}\right)=0 & \text { in }(0, T) \times(0,1), \\ \partial_{t} w_{2}-\partial_{x}\left(\gamma_{2} \partial_{x} w_{2}\right)+a w_{1}=0 & \text { in }(0, T) \times(0,1), \\ w_{1}(t, 1)=w_{2}(t, 1) & \text { in }(0, T), \\ \gamma_{1}(1) \partial_{x} w_{1}(t, 1)+\gamma_{2}(1) \partial_{x} w_{2}(t, 1)+\alpha w_{1}(t, 1)=0 & \text { in }(0, T), \\ w_{1}(0, \cdot)=w_{2}(0, \cdot)=0 & \text { in }(0,1),\end{cases}
$$


along with the conditions at $x=0$ as

$$
w_{1}(t, 0)=\mathcal{B}_{2}^{*} e^{-(T-t) \mathcal{A}_{\alpha, a}^{*} \zeta}, \quad w_{2}(t, 0)=0 \quad \text { in }(0, T) .
$$

Now, recall the definition of $J_{\epsilon}$ from (5.2), and a straightforward computation yields

$$
\nabla J_{\epsilon}(\zeta)=\Lambda \zeta+\epsilon \mathcal{A}_{\alpha} \zeta+e^{-T \mathcal{A}_{\alpha, a}} y_{0}
$$

one can see that $e^{-T \mathcal{A}_{\alpha, a}} y_{0}$ is the (free) solution to $(1.7)-(1.8 \mathrm{~b})$ when we consider $v=0$ in $(1.8 \mathrm{~b})$, and with initial data $y_{0} \in E$.

In this way, the control we are looking for, can be obtained as follows: for any given $\epsilon>0$, we first compute the unique minimizer $\zeta^{\epsilon}$ of $J_{\epsilon}$, that is the unique solution to the linear problem

$$
\epsilon \mathcal{A}_{\alpha} \zeta+\Lambda \zeta=-e^{-T \mathcal{A}_{\alpha, a}} y_{0}
$$

and then determine the solution to the adjoint equation (3.1) by choosing the terminal data $\zeta^{\epsilon}$. Since $\Lambda$ is a symmetric and positive semi-definite operator, the conjugate gradient algorithm is a good candidate to solve the linear problem (5.6). We refer to [30, Ch. 2, Sec. 2.1-2.3] for the implementation of such algorithm.

Once we have computed the minimizer, we use the formula (5.3) to obtain the desired control and by means of Theorem 5.2, the expected controllability properties can be tested by analyzing the involved quantities with respect to the parameter $\epsilon$.

5.2 Numerical implementation for the general interior-boundary coupled system

For the numerical tests, the systems (1.1) (along with the Assumption 1) and its adjoint are discretized in time by using a standard implicit Euler scheme and discretized in space by a finite-difference scheme adapted to the corresponding boundary conditions. Let $N, M \in \mathbb{N}^{*}$, we set $\delta t=T / M$ and $h=1 /(N+1)$ and consider the following discretization for the space and time variables

$$
\begin{gathered}
0=x_{0}<x_{1}<\ldots<x_{N}<x_{N+1}=1, \\
0=t_{0}<t_{1}<\ldots<T_{M}=T,
\end{gathered}
$$

where $x_{i}=i h, i \in[0, N+1]$, and $t_{n}=n \delta t, n \in[0, M]$. With this, we consider fully discrete systems of the form

$$
\left\{\begin{array}{l}
\frac{y^{n+1}-y^{n}}{\delta t}+\mathcal{A}_{h} y^{n+1}=\mathcal{B}_{h} v^{n+1}, \quad n \in\{0, \ldots M-1\}, \\
y^{0}=y_{h}^{0},
\end{array}\right.
$$

where $y=\left(y^{n}\right)_{n \in[0, M]}$ and $v=\left(v^{n}\right)_{n \in[0, M-1]}$ are the (discrete) state and control variables, $y_{h}^{0} \in \mathbb{R}^{2 N}$ is an approximation of the given initial data $y(0, \cdot), \mathcal{A}_{h} \in$ $\mathbb{R}^{2 N \times 2 N}$ is a suitable approximation of the elliptic operator $\mathcal{A}$ and $\mathcal{B}_{h} \in \mathbb{R}^{2 N}$ stands for the corresponding approximation of the control operator. We also choose here constant diffusion coefficients $\gamma_{1}, \gamma_{2} \in \mathbb{R}^{+}$and further we denote $\gamma:=\left(\begin{array}{cc}\gamma_{1} & 0 \\ 0 & \gamma_{2}\end{array}\right) \in$ $M_{2 \times 2}(\mathbb{R})$.

As usual, we denote by $y_{j}(j=1,2)$, each of the components of system (1.1). 
1. Using a standard finite-difference method, we construct the matrix $\mathcal{A}_{h, D} \in$ $\mathbb{R}^{2 N \times 2 N}$, which is composed by two tridiagonal matrix coming from the discretization of the operator $-\gamma_{j} \partial_{x}^{2}, j=1,2$, with homogeneous Dirichlet boundary conditions, that is

$$
\mathcal{A}_{h, D}=\left(\begin{array}{cc}
\mathcal{A}_{h}^{1} & 0 \\
0 & \mathcal{A}_{h}^{2}
\end{array}\right)
$$

where for each $j=1,2,\left(\mathcal{A}_{h}^{j} y_{j}\right)_{i}=-\frac{\gamma_{j}}{h^{2}}\left(y_{j, i+1}-2 y_{j, i}+y_{j, i-1}\right), i=1, \ldots, N$. At this point, we impose that $y_{j, 0}=y_{j, N+1}=0$. In the subsequent steps we will compute and add the contribution of the boundary conditions to the discretization scheme.

2. To incorporate the effect of the boundary conditions (of the system (1.1)) at the left point, we compute

$$
\mathcal{A}_{h, 0}=-\left(-\mathcal{N}_{0} \gamma+h \mathcal{D}_{0}\right)^{-1} \mathcal{N}_{0} \gamma
$$

This corresponds to writing the boundary unknowns $y_{j, 0}$ in terms of the values of $y_{j, 1}$ and yields a $2 \times 2$ matrix. The result will be then used to construct the auxiliary matrix:

$$
\widetilde{\mathcal{A}}_{h}=\mathcal{A}_{h, D}-\frac{1}{h^{2}} \gamma \mathcal{A}_{h, 0} \otimes\left(\begin{array}{cccc}
1 & 0 & \cdots & 0 \\
0 & 0 & \ddots & \vdots \\
\vdots & \ddots & \ddots & 0 \\
0 & \cdots & 0 & 0
\end{array}\right)_{N \times N}
$$

where $\otimes$ denotes the Kronecker product, i.e., for matrices $S \in \mathbb{R}^{m \times n}$ and $T \in \mathbb{R}^{p \times q}$, the product $S \otimes T$ is the $m p \times n q$ matrix given by

$$
S \otimes T=\left(\begin{array}{ccc}
s_{11} T & \cdots & s_{1 n} T \\
\vdots & \ddots & \vdots \\
s_{1 m} T & \cdots & s_{m n} T
\end{array}\right)
$$

3. In a similar fashion, for adding the contribution of the boundary at $x=1$, we compute

$$
\mathcal{A}_{h, 1}=\left(\mathcal{N}_{1} \gamma+h \mathcal{D}_{1}\right)^{-1} \mathcal{N}_{1} \gamma
$$

This will give the coefficients obtained by expressing $y_{j, N+1}$ in terms of the values $y_{j, N}$. We add the resulting matrix to the one obtained in the previous step as follows

$$
\widehat{\mathcal{A}}_{h}=\widetilde{\mathcal{A}}_{h}-\frac{1}{h^{2}} \gamma \mathcal{A}_{h, 1} \otimes\left(\begin{array}{cccc}
0 & 0 & \cdots & 0 \\
0 & \ddots & \ddots & \vdots \\
\vdots & \ddots & 0 & 0 \\
0 & \cdots & 0 & 1
\end{array}\right)_{N \times N}
$$

4. To conclude, we need to add the internal coupling terms. This can be easily done by computing

$$
\mathcal{A}_{h}=\widehat{\mathcal{A}}_{h}+\mathcal{M}_{\text {coup }} \otimes I_{N \times N}
$$


Observe that in our theoretical results, we have considered the simple case where the control $v$ is applied to one of the equations of system (1.7) through the boundary conditions (1.8a) or (1.8b). However, observe that in the general system (1.1), the control can be applied in fact to any linear combination of boundary values. To take into account this in our discretization, we propose the following:

1. We obtain the auxiliary vector

$$
\widetilde{\mathcal{B}}_{h}=h\left(-\mathcal{N}_{0} \gamma+h \mathcal{D}_{0}\right)^{-1} B,
$$

where one might consider $B$ as the canonical vector $(1,0)$ or $(0,1)$, depending on which equation the control is being applied.

2. We obtain the control operator by setting

$$
\mathcal{B}_{h}=\frac{1}{h^{2}} \gamma \widetilde{\mathcal{B}}_{h} \otimes\left(\begin{array}{c}
1 \\
0 \\
\vdots \\
0
\end{array}\right)_{N}
$$

Remark 5.3 Some remarks are in order.

- In the general case, under the conditions of Assumption 1, the invertibility of the matrices shown in formulas (5.9), (5.12) and (5.15) is guaranteed for any $h>0$ small enough, see Lemma A.1 in appendix.

- The discretization of system (1.7) with either boundary conditions (1.8a), (1.8b) is a particular case of the scheme presented above. Indeed, we readily see that for such cases we have $\mathcal{A}_{h, 0}=O_{2 \times 2}$,

$$
\mathcal{A}_{h, 1}=\left(\begin{array}{ll}
\frac{\gamma_{1}}{\gamma_{1}+\gamma_{2}+\alpha h} & \frac{\gamma_{2}}{\gamma_{1}+\gamma_{2}+\alpha h} \\
\frac{\gamma_{1}}{\gamma_{1}+\gamma_{2}+\alpha h} & \frac{\gamma_{2}}{\gamma_{1}+\gamma_{2}+\alpha h}
\end{array}\right),
$$

$\mathcal{M}_{\text {coup }}=\mathcal{M}_{a}$ and $\widetilde{B}_{h}=\left(\begin{array}{l}0 \\ 1\end{array}\right)$ for the boundary condition (1.8a) (resp. $\left(\begin{array}{l}1 \\ 0\end{array}\right)$ for $(1.8 \mathrm{~b}))$. In this case, we note that since $\alpha \geq 0$, (5.17) holds for any value of $h>0$.

We denote by $E_{h}, \mathcal{H}_{-\alpha, h}, U_{h}$ and $L_{\delta t}^{2}\left(0, T ; U_{h}\right)$ the discrete spaces associated to $E, \mathcal{H}_{-\alpha}, \mathbb{R}$ and $L^{2}(0, T ; \mathbb{R})$, respectively. We denote by $F_{\epsilon}^{h, \delta t}$ the discretization of the functional $F_{\epsilon}, v^{\epsilon, h, \delta t}$ the corresponding minimizer and $y^{\epsilon, h, \delta t}=\left(y_{1}^{\epsilon, h, \delta t}, y_{2}^{\epsilon, h, \delta t}\right)$ the associated controlled solution.

As usual in this context, to connect the discretization to the control problem, we use the penalization parameter $\epsilon=\phi(h)=h^{4}$. This choice is consistent with the order of approximation of the finite difference scheme. We refer the reader to [14, Section 4] for a more detailed discussion on the selection of the function $\phi(h)$ in the context of the null-controllability of some parabolic problems and its implications.

To concentrate on the dependency of the numerical experiments with respect to the mesh size $h$, in the following we will always set $M=4000$. This is due to the fact that the results do not depend too much on the time step (as soon as it is chosen to ensure at least the same accuracy as the space discretization). This was observed in [14] and the same still applies here. 
5.3 Numerical experiments

\subsubsection{Dirichlet boundary control}

The case $a=0$. Using our computational tool, we begin by obtaining the solution to system (1.7)-(1.8b) without any control. We consider the set of parameters

$$
\begin{gathered}
T=0.4, \quad \gamma_{1}=\gamma_{2}=1 \\
a=0, \quad \alpha=1 \\
y_{0,1}(x)=\sin (\pi x), \quad y_{0,2}(x)=\mathbf{1}_{(0.3,0.8)}(x),
\end{gathered}
$$

and plot the time evolution of the uncontrolled system in Figure 5.1. We observe that the solution of both components is damped over time, however they are far from the desired null target.

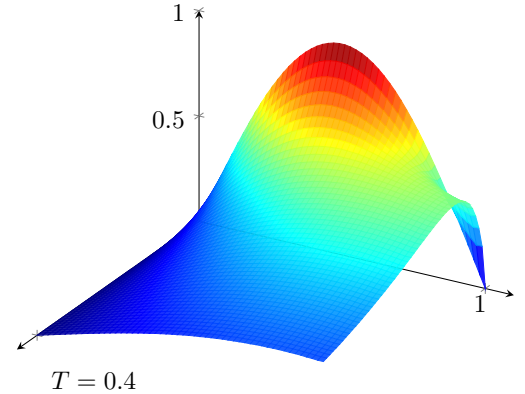

(a) The state $(t, x) \mapsto y_{1}(t, x)$

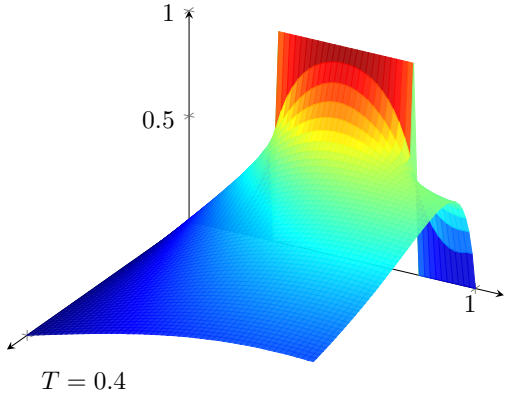

(b) The state $(t, x) \mapsto y_{2}(t, x)$

Fig. 5.1 Evolution in time of the uncontrolled solution of system (1.7)-(1.8b) for $a=0, \alpha=1$.

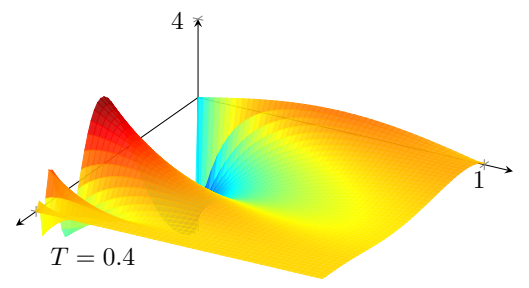

(a) The state $(t, x) \mapsto y_{1}(t, x)$

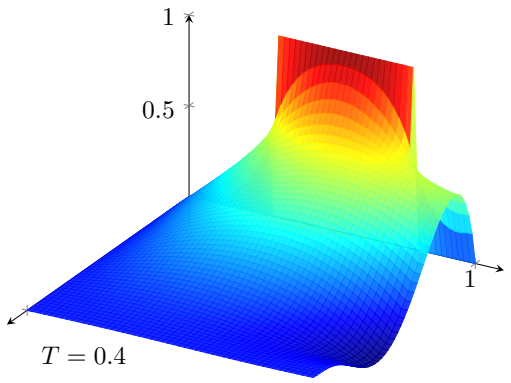

(b) The state $(t, x) \mapsto y_{2}(t, x)$

Fig. 5.2 Evolution in time of the controlled solution of system (1.7)-(1.8b) for $a=0, \alpha=1$.

In Figure 5.2, we show the solution $\left(y_{1}, y_{2}\right)$ obtained after applying the HUM control $v(t)$ (see Figure 5.3) computed by algorithm (5.5)-(5.6). We observe, that due to this action, both components reach zero at the prescribed time $T=0.4$. 
Notice that, since we have chosen $a=0$ in $(1.7)-(1.8 \mathrm{~b})$, the action of the control acts indirectly on the second component just by means of the boundary coupling. Intuitively, this problem is harder to solve than other classical problems where the coupling is made in the internal domain.

As far as the asymptotic of the method, we present in Figure 5.4 the behavior of various quantities of interest as the mesh size goes to 0 . We observe that the control cost $\left\|v_{\phi(h)}^{h, \delta t}\right\|_{L_{\delta t}^{2}\left(0, T ; U_{h}\right)}(\smile-)$ as well as the optimal energy inf $F_{\phi(h)}^{h, \delta t}(-\bullet)$ remain bounded as the mesh size $h$ tends to 0 . Also, we see that the norm of the state $\left\|\left(y_{1}^{h, \delta t}(T), y_{2}^{h, \delta t}(T)\right)\right\|_{\mathcal{H}_{-\alpha, h}}(--)$ behaves like $\sim C \sqrt{\phi(h)}=C h^{2}$. This behavior is in agreement with Theorem 5.2 and illustrates our null controllability result.

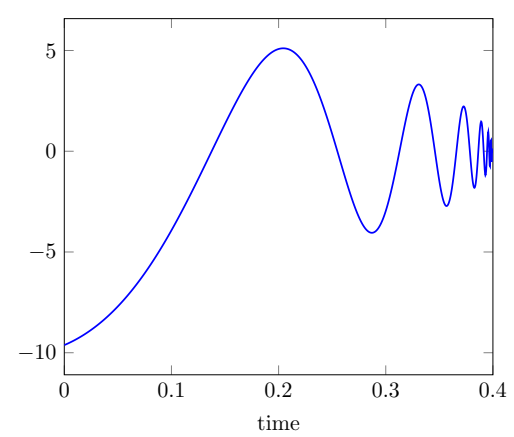

Fig. 5.3 Control function $v(t)$.

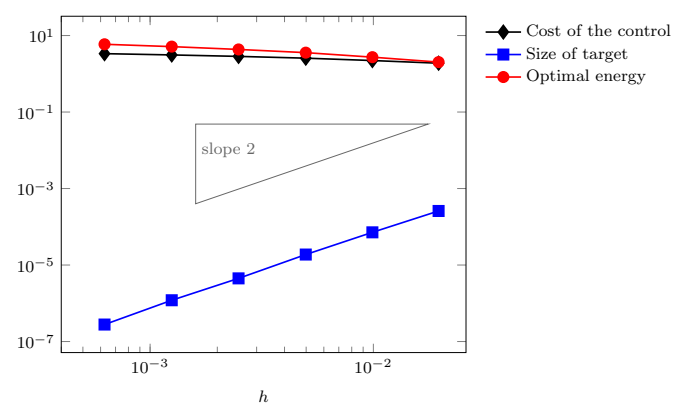

Fig. 5.4 Convergence properties using the penalized HUM technique, the case $a=0, \alpha=1$ (Dirichlet control case).

The case $a \neq 0$. According to our main controllability results: Theorems 1.2 and 1.3 , the controllability of system (1.7) is guaranteed depending on the selection of the parameters $(\alpha, a)$ and the way the control enters the system. When the control enters through the first component, that is, when $(1.8 \mathrm{~b})$ is verified, we know from Lemma 4.7 that there exist values of $(\alpha, a)$ for which system (1.7) is not even approximately controllable, this is described by means of the set $\mathcal{R}$ 
defined in (4.29). We illustrate this fact below. By using a numerical algorithm, we can determine that the approximate pair $\left(\alpha_{0}, a_{0}\right)=(1,3.1931469)$ belongs to $\mathcal{R}$ (see Figure 4.2) and corresponds to the critical eigenvalue $\lambda_{\alpha_{0}, a_{0}}^{c} \approx 5.7421936$. Therefore, the eigenfunction $\Phi_{\lambda_{\alpha_{0}, a_{0}}^{c}}$ fails to verify the Fattorini-Hautus criterion.

In Figure 5.5, we plot the eigenfunction corresponding to the critical eigenvalue $\lambda_{\alpha_{0}, a_{0}}^{c}$. We observe that the first component of the eigenfunction, that is the one in blue color, is almost flat as it approaches to the boundary point $x=0$ and in fact, numerically we can compute the size of the normal derivative which is of order $10^{-5}$. We expect that this is somehow reflected during the penalized HUM procedure.

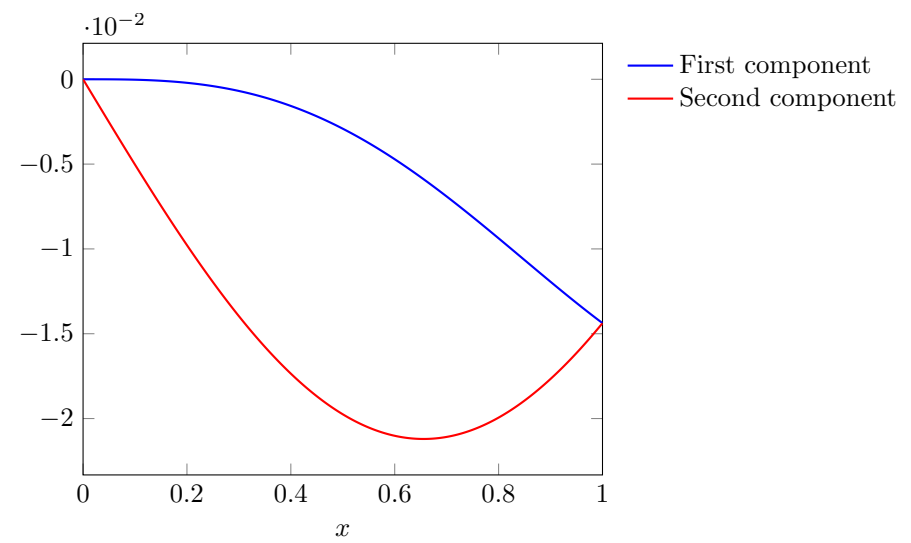

Fig. 5.5 The eigenfunction $\Phi_{\lambda_{\alpha_{0}, a_{0}}^{c}}$ corresponding to the critical eigenvalue $\lambda_{\alpha_{0}, a_{0}}^{c}$ (Dirichlet case).

We set the parameters

$$
\begin{gathered}
T=0.3, \quad \gamma_{1}=\gamma_{2}=1, \\
a_{0}=3.1931469, \quad \alpha_{0}=1, \\
y_{0,1}(x)=10 \sin (2 \pi x)^{3}, \quad y_{0,2}(x)=5 \times \mathbf{1}_{(0.3,0.8)}(x),
\end{gathered}
$$

and apply our computational tool to obtain boundary controls. In Figure 5.6a we observe the asymptotic behavior of the algorithm. Unlike the previous case, we observe that the optimal energy (--) blows up as $\phi(h)^{-1}=h^{4}$ while the size of the target $(--)$ remains constant. This indicates that for the above selection of the initial data (under the consideration of the critical pair), our system (1.7)(1.8b) is neither approximately nor null-controllable, which is in accordance with our theoretical results.

A further validation of this result can be done by adapting [14, Theorem 1.11], which gives a hint of the general behavior of the penalized HUM approach in the limit. In our case, it can be shown that as $h \rightarrow 0, \mathcal{A}_{\alpha, h}^{-1} y^{h}(T)$ should converge towards a nonzero function which belongs to the space of unobservable modes; as we have seen in Section 4.2.1, the span set of such space consists only one element which is the eigenfunction associated to the critical eigenvalue. Thus, we expect to see this at the numerical level. 
In Figure 5.7, it can be seen that as $N$ increases (and therefore $h \downarrow 0$ ) the target is converging towards some function instead of going to zero. In this case, it is clear that the target converges to the critical eigenfunction (up to a constant) shown in Figure 5.5 which validates the discussion above.

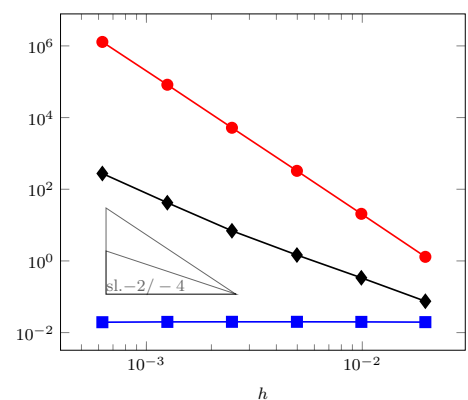

(a) Control applied on $y_{1}((1.7)-$ $(1.8 \mathrm{~b}))$

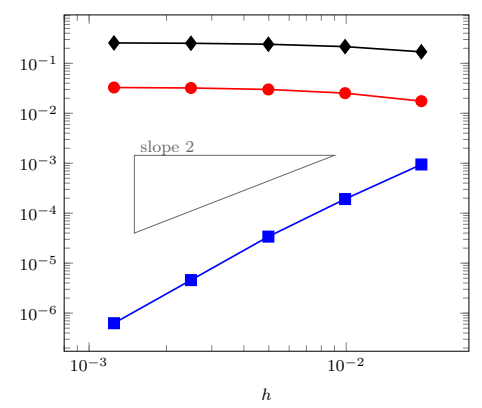

(b) Control applied on $y_{2}((1.7)-$ $(1.8 \mathrm{a}))$

Fig. 5.6 Convergence properties of the HUM technique for the critical pair $\left(\alpha_{0}, a_{0}\right)$ with Dirichlet control applied on different components. Same legend as in Figure 5.4.

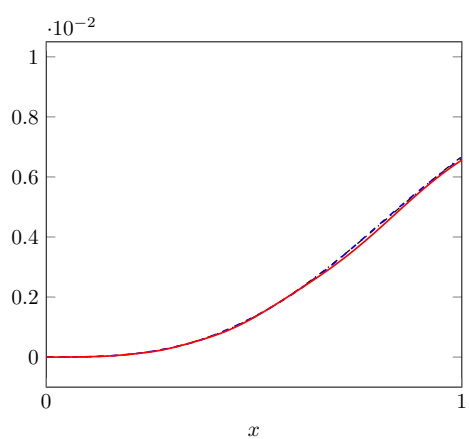

(a) $\mathcal{A}_{\alpha, h}^{-1} y_{1}^{h}(T)$

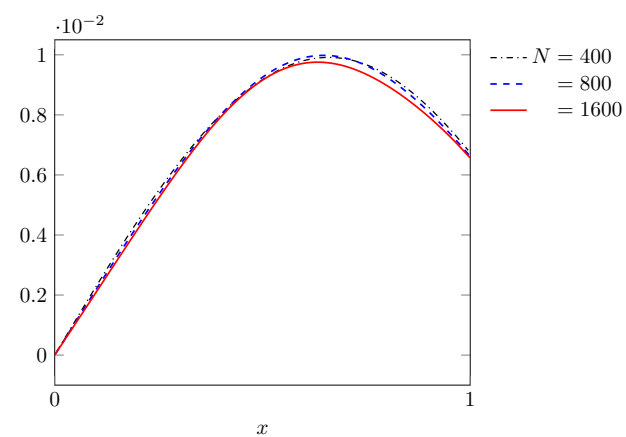

(b) $\mathcal{A}_{\alpha, h}^{-1} y_{2}^{h}(T)$

Fig. 5.7 Convergence of the target as $h \downarrow 0$ for the critical pair $\left(\alpha_{0}, a_{0}\right)=(1,3.1931469)$ for the system $(1.7)-(1.8 \mathrm{~b})$.

At this point, we shall mention that the approximation of the critical parameter $a_{0}$ plays an important role in the numerical experiments. In Figure 5.8 we present a series of experiments where the parameter $a_{0}$ is approximated by truncating up to a certain number of decimals. For a fixed value of $h$, We see that for a rough approximation (two decimals) the convergence of the target is not as good as for the finer ones (in the experiments shown $h=1 / 1600$ ). We recall that the critical parameters come from obtaining a simultaneous solution to (4.28), therefore the non-controllability result is very sensitive w.r.t. even small changes of such values. The behavior shown in Figure 5.8 is therefore consistent with this fact. 


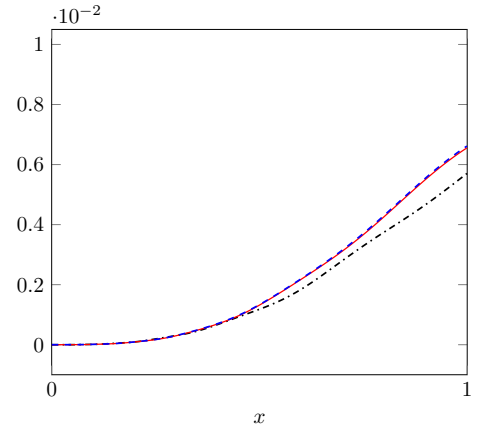

(a) $\mathcal{A}_{\alpha, h}^{-1} y_{1}^{h}(T)$

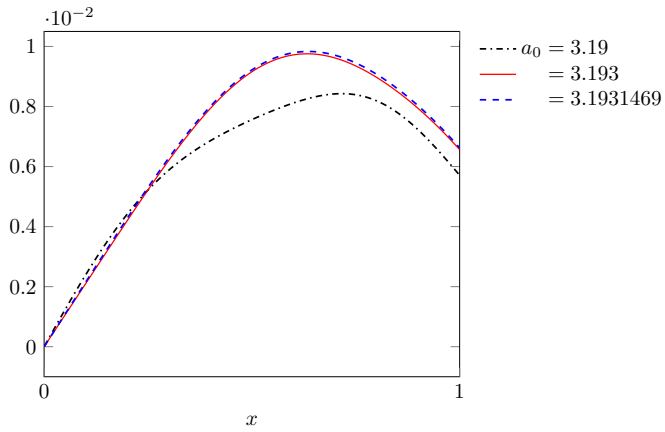

(b) $\mathcal{A}_{\alpha, h}^{-1} y_{2}^{h}(T)$

Fig. 5.8 Convergence of the target for $\alpha_{0}=1$ and different approximations of $a_{0}$ for the system (1.7)-(1.8b).

We finish the discussion here by emphasizing that the behavior shown in Figure 5.6 a comes from the fact that the control is placed on the boundary of the first component, namely the condition (1.8b). If instead we consider the boundary control on the second component as (1.8a), Theorem 1.2 indicates that regardless the choice of $(\alpha, a) \in \mathbb{R}_{0}^{+} \times \mathbb{R}$, system (1.7)-(1.8a) is null-controllable at any time $T$. We illustrate this fact in Figure $5.6 \mathrm{~b}$, where consider the same parameters as in (5.21) with the difference that the control is applied on the boundary of the second equation. We observe that as $h \rightarrow 0$ the size of the target decreases as $\sqrt{\phi(h)}=h^{2}$ and both the control cost and the optimal energy remain bounded, which is in concordance with the theoretical controllability result.

\subsubsection{Neumann boundary control}

The goal of this section is to show that our computational tool can be used to illustrate other cases not covered in the theoretical results presented in this paper. This is possible, thanks to the general methodology we introduced in Section 5.2.

We will discuss about the controllability of system (1.7) in the case when the boundary conditions at $x=0$ are replaced by the Neumann conditions

$$
\begin{array}{rlll}
\text { either } & \partial_{x} y_{1}(t, 0)=0, \quad \gamma_{2} \partial_{x} y_{2}(t, 0)=v(t) & \text { in }(0, T), \\
\text { or } & \gamma_{1} \partial_{x} y_{1}(t, 0)=v(t), \quad \partial_{x} y_{2}(t, 0)=0 & \text { in }(0, T),
\end{array}
$$

where $\gamma_{1}, \gamma_{2}$ are chosen as positive constants.

The well-posedness of this kind of system can be drawn almost in a similar approach as we did for the Dirichlet cases.

For later use, we write down the observation operator corresponding to the second system (1.7)-(5.22b) as follows

$$
\widetilde{\mathcal{B}}^{*}: u=\left(u_{1}, u_{2}\right) \in\left(H^{1}(0,1)\right)^{2} \mapsto u_{1}(0) .
$$

For the above Neumann control systems (1.7)-(5.22), one could again play with the Carleman technique and/or a rigorous study of the spectral analysis (which is 
at the heart of the moments approach), but we shall not pursue any detailed study regarding those in this paper.

However, at the numerical level, using the discretization scheme shown in Section 5.2 , we just have to set the matrices

$$
\begin{array}{ll}
\mathcal{N}_{0}=\left(\begin{array}{ll}
1 & 0 \\
0 & 1
\end{array}\right), & \mathcal{D}_{0}=\left(\begin{array}{ll}
0 & 0 \\
0 & 0
\end{array}\right), \\
\mathcal{N}_{1}=\left(\begin{array}{ll}
0 & 0 \\
1 & 1
\end{array}\right), & \mathcal{D}_{1}=\left(\begin{array}{cc}
1 & -1 \\
\alpha & 0
\end{array}\right),
\end{array}
$$

and compute the formulas given in (5.8)-(5.16).

This simple idea actually allows to test for many configurations and different values of $a$ and $\alpha$.

We first consider the following simulation parameters

$$
\begin{gathered}
T=0.5, \quad \gamma_{1}=\gamma_{2}=1, \\
a=2, \quad \alpha=4, \\
y_{0,1}(x)=\sin (\pi x), \quad y_{0,2}(x)=\mathbf{1}_{(0.3,0.8)}(x),
\end{gathered}
$$

and use our tool to obtain numerical results for two different configurations. In Figure 5.9a, we show the convergence result for the case where $v$ is applied on the first component, that is, $(5.22 \mathrm{~b})$. We can see that as $h$ tends to zero, the size of the target decreases as $\sqrt{\phi(h)}=h^{2}$ and both the optimal energy and the control cost remain bounded.

On the other hand, we show the result in Figure $5.9 \mathrm{~b}$ by changing the control to the second component, i.e., we consider (5.22a).

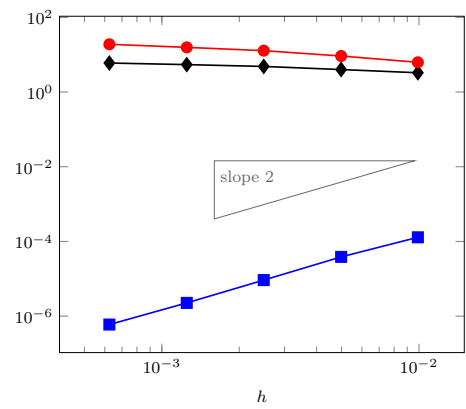

(a) Control applied on the first component

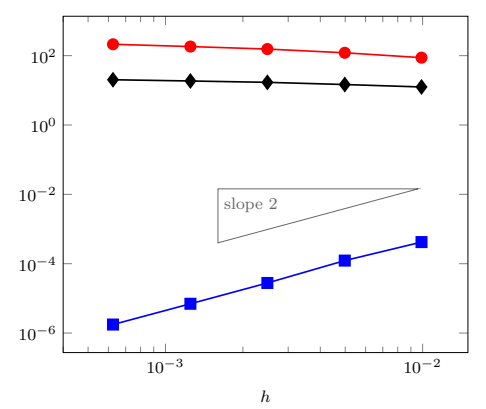

(b) Control applied on the second component

Fig. 5.9 Convergence properties of the HUM technique with Neumann control applied in different equations for $a=2, \alpha=4$ (the systems (1.7)-(5.22)). Same legend as in Figure 5.4.

Both the simulations point toward a positive null controllability result, nevertheless one should be cautious with such conclusion. In fact, in the case where the control is applied on the component $y_{2}$, we expect that some adaptations can be made to our Carleman estimate presented in Theorem 3.2 to deduce a similar result for (1.7)-(5.22a), and thus one can expect null-controllability for any $a$ and $\alpha$. 
On the other hand, alike the Dirichlet case (1.7)-(1.8b), we suspect that a detailed analysis of the adjoint elliptic operator associated with the Neumann case (1.7)-(5.22b) is essential to answer the controllability question.

Although we will not give any theoretical details, the numerical evidence presented in Figure 5.10 shows that as in the Dirichlet case, there exists at least one couple $(\alpha, a)$ for which the observation of one eigenfunction is 0 . We numerically find some pair (approximate value) $\left(\alpha_{c}, a_{c}\right)=(0.1,1.2369289)$. In Figure 5.11 we are plotting the first eigenfunction associated to this pair and from there, it is clear that such eigenfunction is non-observable (see the def. of observation operator $\widetilde{\mathcal{B}}^{*}$ in $(5.23))$.

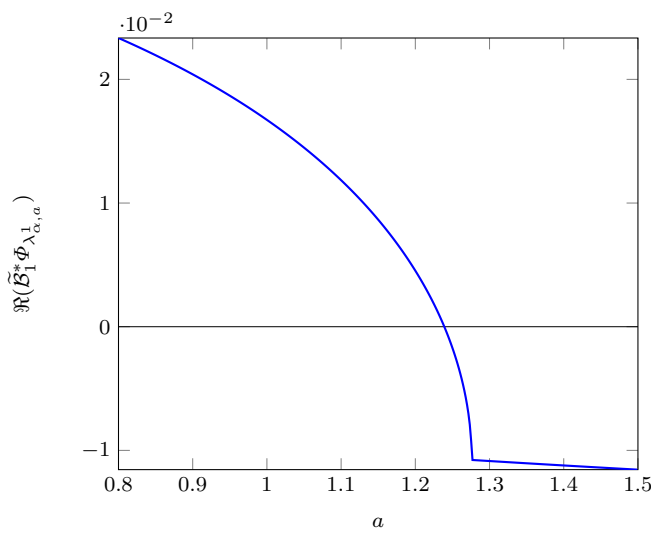

Fig. 5.10 Size of the observations w.r.t. the first eigenfunctions $\Phi_{\lambda_{\alpha, a}^{1}}$, in the Neumann case (1.7)-(5.22b) for $\alpha=0.1$ and $a \in[0.8,3]$.

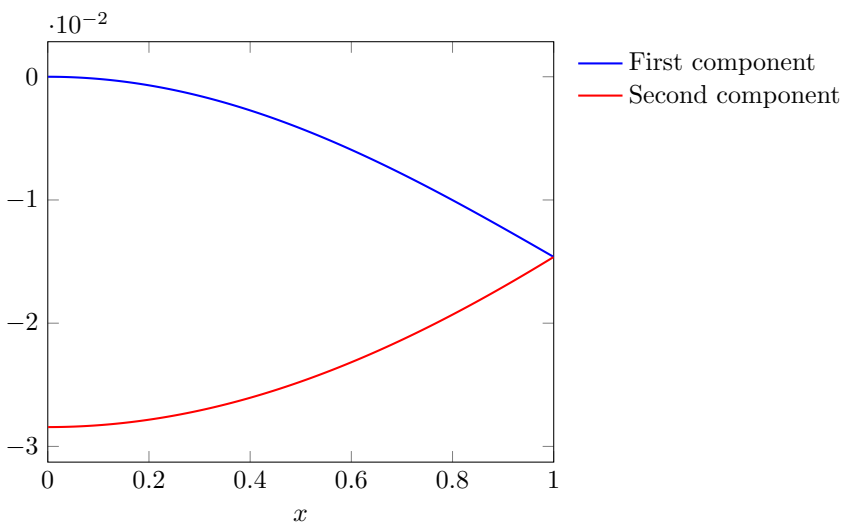

Fig. 5.11 The eigenfunction $\Phi_{\lambda_{\alpha_{c}, a_{c}}^{1}}$ corresponding to the critical eigenvalue (the first one) $\lambda_{\alpha_{c}, a_{c}}^{1}$ (Neumann case). 
We use this new couple $\left(\alpha_{c}, a_{c}\right)$ for some simulation purposes. In Figure 5.12a, we present the convergence properties w.r.t. to the penalized HUM approach and as in the Dirichlet case we observe that the size of the target is not decreasing while the optimal energy and cost of the control are blowing up. This points towards a non-controllability result.

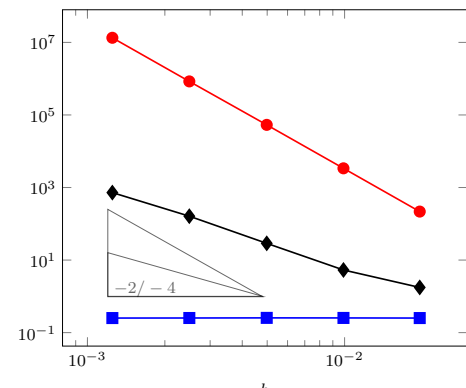

(a) Control applied on $y_{1}((1.7)-$ $(5.22 \mathrm{~b}))$

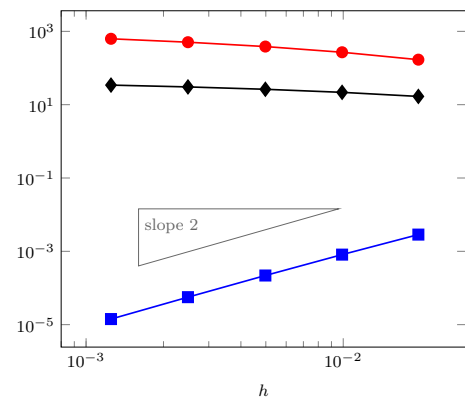

(b) Control applied on $y_{2}((1.7)-$ $(5.22 \mathrm{a}))$

Fig. 5.12 Convergence properties w.r.t. HUM approach for the critical pair $\left(\alpha_{c}, a_{c}\right)$ with Neumann control applied on different components.

Following with the discussion of the Dirichlet case, we see in Figure 5.13 that the target is indeed converging towards the critical eigenfunction (up to some constant) which is consistent with the lack of controllability. In view of these results, a deeper study of the Neumann control case is needed to conclude.

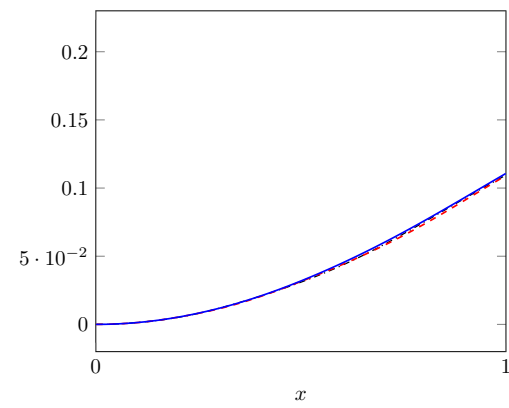

(a) First component of the target

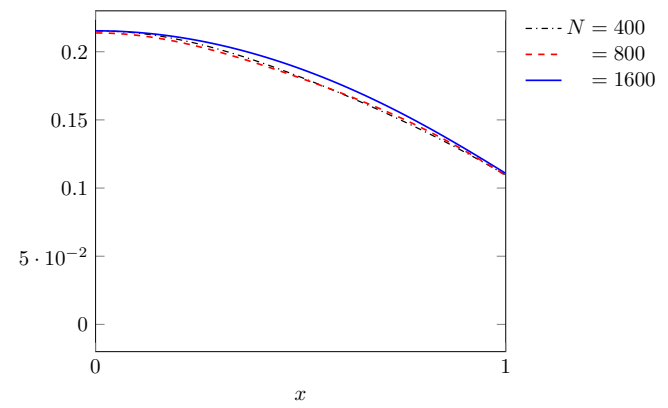

(b) Second component of the target

Fig. 5.13 Convergence of the target as $h \downarrow 0$ for the critical values $\left(\alpha_{c}, a_{c}\right)=(0.1,1.2369289)$ for the system (1.7)-(5.22b).

We would like to emphasize now that as in the Dirichlet case, we need a good approximation of the critical parameter $a_{c}$ to observe the lack of controllability of the system. In Figure 5.14, we see the convergence of the target for $h=1 / 1600$ and different approximations of $a_{c}$. This experiment seems to be more sensitive than the previous case since we need at least four decimal approximation (for the 
Dirichlet case, it was three; see Fig. 5.8) of the parameter $a_{c}$ (precisely, 1.2369) to obtain some convergence of the target for the given value of $h$.

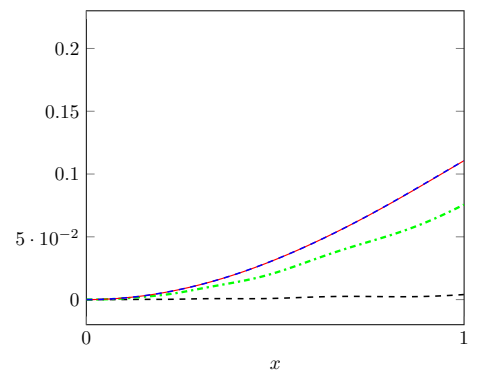

(a) First component of the target

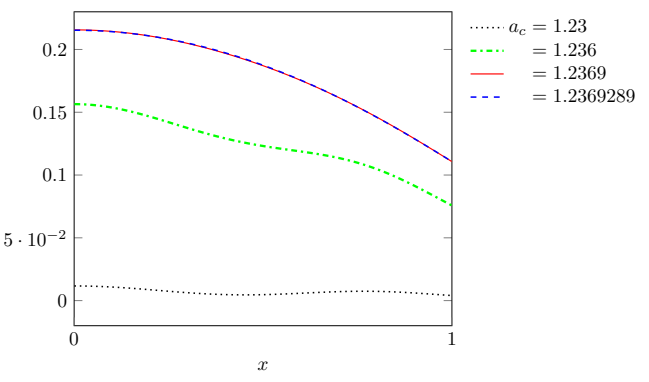

(b) Second component of the target

Fig. 5.14 Convergence of the target for $\alpha_{c}=0.1$ and different approximations of $a_{c}$ for the system (1.7)-(5.22b).

We finish this part by placing an experimental result for the same critical pair $\left(\alpha_{c}, a_{c}\right)$ but the control we exert on the second component instead of the first one, i.e., for the system (1.7)-(5.22a). In contrast of the case (1.7)-(5.22b), we obtain here nice behavior of the target size, control cost and optimal energy (see Figure 5.12b), which shows a positive hope of the null-controllability of the system (1.7)-(5.22a), at least from the numerical point of view.

\section{A An intermediate result}

Lemma A.1 Let $\mathcal{D}$ and $\mathcal{N}$ be two real $d \times d$ matrices such that

$$
(\mathcal{D}, \mathcal{N}) \text { is full rank, }
$$

and

$$
\mathcal{D} \mathcal{N}^{*} \text { is self adjoint, }
$$

then $\mathcal{N}+t \mathcal{D}$ is invertible for any $t \in \mathbb{R}$ except perhaps for a finite number of values of $t$.

Proof We follow the same computations as in [10, Theorem 1.4.4]. More precisely, we first observe that, under the assumptions of the lemma, we have that $\mathcal{D}+i \mathcal{N}$ is invertible. Indeed, - by (A.1), we know that $\left(\operatorname{ker} \mathcal{D}^{*}\right) \cap\left(\operatorname{ker} \mathcal{N}^{*}\right)=\{0\}$,

- by (A.2), for any $x \in \mathbb{C}^{2}$ we have

$$
\left\|\left(\mathcal{D}^{*}-i \mathcal{N}^{*}\right) x\right\|^{2}=\left\|\mathcal{D}^{*} x\right\|^{2}+\left\|\mathcal{N}^{*} x\right\|^{2},
$$

so that $\operatorname{ker}\left(\mathcal{D}^{*}-i \mathcal{N}^{*}\right) \subset\left(\operatorname{ker} \mathcal{D}^{*}\right) \cap\left(\operatorname{ker} \mathcal{N}^{*}\right)=\{0\}$ and the claim is proved

We can now can define $\mathcal{U}=-(\mathcal{D}+i \mathcal{N})^{-1}(\mathcal{D}-i \mathcal{N})$ (which is actually a unitary matrix but we don't need this fact here). It satisfies

$$
\begin{gathered}
2(\mathcal{D}+i \mathcal{N})^{-1} \mathcal{D}=(\mathcal{D}+i \mathcal{N})^{-1}(\mathcal{D}+i \mathcal{N}+\mathcal{D}-i \mathcal{N})=(I-\mathcal{U}) \\
2(\mathcal{D}+i \mathcal{N})^{-1} \mathcal{N}=-i(\mathcal{D}+i \mathcal{N})^{-1}((\mathcal{D}+i \mathcal{N})-(\mathcal{D}-i \mathcal{N}))=-i(I+\mathcal{U})
\end{gathered}
$$

If we assume that $t \in \mathbb{R}$ is such that $\mathcal{N}+t \mathcal{D}$ is not invertible, then there exists $x \in \mathbb{R}^{d}, x \neq 0$ such that $(\mathcal{N}+t \mathcal{D}) x=0$. Left-multiplying this equality by $2(\mathcal{D}+i \mathcal{N})^{-1}$ an using the above relations we end up with

$$
\left(\frac{t-i}{t+i} I-\mathcal{U}\right) x=0
$$

which proves that $(t-i) /(t+i)$ is an eigenvalue of $\mathcal{U}$. This can only happen for a finite number of values of $t$. 


\section{Acknowledgment}

The authors would like to thank both referees for their very careful reading of the first version of this paper. Their comments have helped us to improve the contents and the presentation of the results.

\section{References}

1. Fatiha Alabau-Boussouira and Matthieu Léautaud. Indirect controllability of locally coupled wave-type systems and applications. Journal de Mathématiques Pures et Appliquées, 99(5):544-576, May 2013.

2. Damien Allonsius and Franck Boyer. Boundary null-controllability of semi-discrete coupled parabolic systems in some multi-dimensional geometries. Mathematical Control and Related Fields, 10(2):217-256, 2020.

3. F. Ammar-Khodja, A. Benabdallah, M. González-Burgos, and L. de Teresa. The Kalman condition for the boundary controllability of coupled parabolic systems. Bounds on biorthogonal families to complex matrix exponentials. J. Math. Pures Appl. (9), 96(6):555590, 2011.

4. Farid Ammar-Khodja, Assia Benabdallah, Manuel González-Burgos, and Luz de Teresa. Recent results on the controllability of linear coupled parabolic problems: a survey. Math. Control Relat. Fields, 1(3):267-306, 2011.

5. Farid Ammar-Khodja, Franz Chouly, and Michel Duprez. Partial null controllability of parabolic linear systems. Math. Control Relat. Fields, 6(2):185-216, 2016.

6. Claudio Amovilli, Frederik E. Leys, and Norman H. March. Electronic energy spectrum of two-dimensional solids and a chain of $\mathrm{C}$ atoms from a quantum network model. J. Math. Chem., 36(2):93-112, 2004.

7. Sergei Avdonin. Control problems on quantum graphs. In Analysis on graphs and its applications, volume 77 of Proc. Sympos. Pure Math., pages 507-521. Amer. Math. Soc., Providence, RI, 2008.

8. Assia Benabdallah, Franck Boyer, Manuel González-Burgos, and Guillaume Olive. Sharp estimates of the one-dimensional boundary control cost for parabolic systems and application to the $N$-dimensional boundary null controllability in cylindrical domains. SIAM Journal on Control and Optimization, 52(5):2970-3001, jan 2014.

9. Assia Benabdallah, Yves Dermenjian, and Jérôme Le Rousseau. Carleman estimates for the one-dimensional heat equation with a discontinuous coefficient and applications to controllability and an inverse problem. J. Math. Anal. Appl., 336(2):865-887, 2007.

10. Gregory Berkolaiko and Peter Kuchment. Introduction to quantum graphs, volume 186 of Mathematical Surveys and Monographs. American Mathematical Society, Providence, RI, 2013.

11. Kuntal Bhandari. Boundary controllability of some coupled parabolic systems with Robin or Kirchhoff conditions. PhD thesis, Institut de Mathématiques de Toulouse, Université Paul Sabatier, September 2020.

12. Kuntal Bhandari and Franck Boyer. Boundary null-controllability of coupled parabolic systems with Robin conditions. Evolution Equations and Control Theory, 10(1):61-102, march 2021.

13. Umberto Biccari and Víctor Hernández-Santamaría. Controllability of a one-dimensional fractional heat equation: theoretical and numerical aspects. IMA J. Math. Control Inform., 36(4):1199-1235, 2019.

14. Franck Boyer. On the penalised HUM approach and its applications to the numerical approximation of null-controls for parabolic problems. In CANUM 2012, 41e Congrès National d'Analyse Numérique, volume 41 of ESAIM Proc., pages 15-58. EDP Sci., Les Ulis, 2013.

15. Franck Boyer, Víctor Hernández-Santamaría, and Luz de Teresa. Insensitizing controls for a semilinear parabolic equation: a numerical approach. Math. Control Relat. Fields, 9(1):117-158, 2019.

16. Stefano Cardanobile and Delio Mugnolo. Analysis of a FitzHugh-Nagumo-Rall model of a neuronal network. Mathematical methods in the applied sciences, 30(18):2281-2308, 2007.

17. Cristian M. Cazacu, Liviu I. Ignat, and Ademir F. Pazoto. Null-controllability of the linear Kuramoto-Sivashinsky equation on star-shaped trees. SIAM J. Control Optim., 56(4):2921-2958, 2018. 
18. Eduardo Cerpa, Emmanuelle Crépeau, and Claudia Moreno. On the boundary controllability of the Korteweg-de Vries equation on a star-shaped network. IMA J. Math. Control Inform., 37(1):226-240, 2020.

19. Eduardo Cerpa, Emmanuelle Crépeau, and Julie Valein. Boundary controllability of the Korteweg-de Vries equation on a tree-shaped network. Evolution Equations and Control Theory (EECT), 2019.

20. Jean-Michel Coron. Control and nonlinearity, volume 136 of Mathematical Surveys and Monographs. American Mathematical Society, Providence, RI, 2007.

21. René Dáger. Approximate controllability of coupled 1-d wave equations on star-shaped graphs. C. R. Math. Acad. Sci. Paris, 354(8):778-782, 2016.

22. René Dáger and Enrique Zuazua. Wave propagation, observation and control in 1-d flexible multi-structures, volume 50 of Mathématiques \&s Applications (Berlin). Springer-Verlag, Berlin, 2006.

23. Ivar Ekeland and Roger Témam. Convex analysis and variational problems, volume 28 of Classics in Applied Mathematics. Society for Industrial and Applied Mathematics (SIAM), Philadelphia, PA, english edition, 1999. Translated from the French.

24. Lawrence C. Evans. Partial differential equations, volume 19 of Graduate Studies in Mathematics. American Mathematical Society, Providence, RI, second edition, 2010.

25. H. O. Fattorini. Some remarks on complete controllability. SIAM J. Control, 4:686-694, 1966.

26. Enrique Fernández-Cara, Manuel González-Burgos, and Luz de Teresa. Boundary controllability of parabolic coupled equations. J. Funct. Anal., 259(7):1720-1758, 2010.

27. Enrique Fernández-Cara, Manuel González-Burgos, Sergio Guerrero, and Jean-Pierre Puel. Null controllability of the heat equation with boundary Fourier conditions: the linear case. ESAIM Control Optim. Calc. Var., 12(3):442-465, 2006.

28. Enrique Fernández-Cara and Sergio Guerrero. Global Carleman inequalities for parabolic systems and applications to controllability. SIAM J. Control Optim., 45(4):1399-1446, 2006.

29. A. V. Fursikov and O. Yu. Imanuvilov. Controllability of evolution equations, volume 34 of Lecture Notes Series. Seoul National University, Research Institute of Mathematics, Global Analysis Research Center, Seoul, 1996.

30. Roland Glowinski, Jacques-Louis Lions, and Jiwen He. Exact and approximate controllability for distributed parameter systems, volume 117 of Encyclopedia of Mathematics and its Applications. Cambridge University Press, Cambridge, 2008.

31. Tosio Kato. Perturbation theory for linear operators. Classics in Mathematics. SpringerVerlag, Berlin, 1995. Reprint of the 1980 edition.

32. Vadim Kostrykin, Jürgen Potthoff, and Robert Schrader. Contraction semigroups on metric graphs. In Analysis on graphs and its applications, volume 77 of Proc. Sympos. Pure Math., pages 423-458. Amer. Math. Soc., Providence, RI, 2008.

33. Vadim Kostrykin and Robert Schrader. Kirchhoff's rule for quantum wires. Journal of Physics A: Mathematical and General, 32(4):595, 1999.

34. Jérôme Le Rousseau and Gilles Lebeau. On Carleman estimates for elliptic and parabolic operators. Applications to unique continuation and control of parabolic equations. ESAIM Control Optim. Calc. Var., 18(3):712-747, 2012.

35. J.-L. Lions. Exact controllability, stabilization and perturbations for distributed systems. SIAM Rev., 30(1):1-68, 1988.

36. J.-L. Lions and E. Magenes. Non-homogeneous boundary value problems and applications. Vol. II. Springer-Verlag, New York-Heidelberg, 1972. Translated from the French by P. Kenneth, Die Grundlehren der mathematischen Wissenschaften, Band 182.

37. G. Lumer. Connecting of local operators and evolution equations on networks. In Potential theory, Copenhagen 1979 (Proc. Colloq., Copenhagen, 1979), volume 787 of Lecture Notes in Math., pages 219-234. Springer, Berlin, 1980.

38. A. S. Markus. Introduction to the spectral theory of polynomial operator pencils, volume 71 of Translations of Mathematical Monographs. American Mathematical Society, Providence, RI, 1988. Translated from the Russian by H. H. McFaden, Translation edited by Ben Silver, With an appendix by M. V. Keldysh.

39. Guillaume Olive. Boundary approximate controllability of some linear parabolic systems. Evol. Equ. Control Theory, 3(1):167-189, 2014.

40. El Maati Ouhabaz. Analysis of heat equations on domains, volume 31 of London Mathematical Society Monographs Series. Princeton University Press, Princeton, NJ, 2005. 
41. Marius Tucsnak and George Weiss. Observation and control for operator semigroups. Birkhäuser Advanced Texts: Basler Lehrbücher. [Birkhäuser Advanced Texts: Basel Textbooks]. Birkhäuser Verlag, Basel, 2009. 